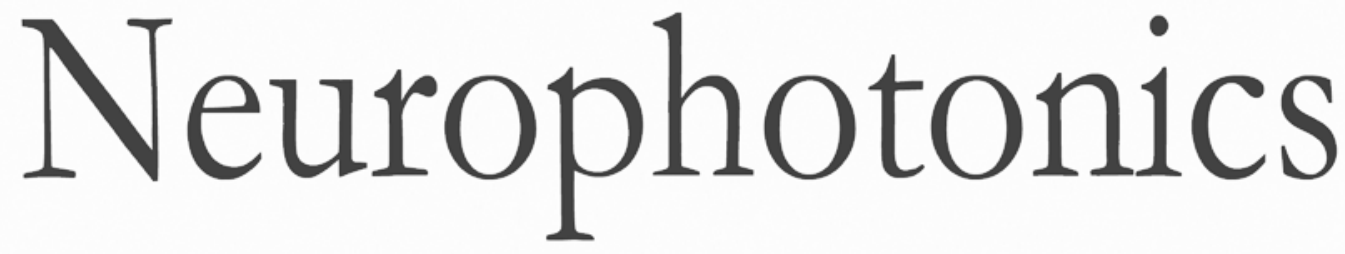

\title{
Investigation of the sensitivity of functional near-infrared spectroscopy brain imaging to anatomical variations in 5- to 11-year-old children
}

\author{
Ashley C. Whiteman \\ Hendrik Santosa \\ Daniel F. Chen \\ Susan Perlman \\ Theodore Huppert
}




\title{
Investigation of the sensitivity of functional near-infrared spectroscopy brain imaging to anatomical variations in 5- to 11-year-old children
}

\author{
Ashley C. Whiteman, ${ }^{a}$ Hendrik Santosa, ${ }^{a}$ Daniel F. Chen, ${ }^{a}$ Susan Perlman, ${ }^{b}$ and Theodore Huppert ${ }^{a, c, \star}$ \\ aUniversity of Pittsburgh, Department of Radiology, Pittsburgh, Pennsylvania, United States \\ bUniversity of Pittsburgh, Department of Psychiatry, Pittsburgh, Pennsylvania, United States \\ 'University of Pittsburgh, Department of Bioengineering, Pittsburgh, Pennsylvania, United States
}

\begin{abstract}
Functional near-infrared spectroscopy (fNIRS) is a noninvasive brain imaging technique that uses scalp-placed light sensors to measure evoked changes in cerebral blood oxygenation. The portability, low overhead cost, and ability to use this technology under a wide range of experimental environments make fNIRS wellsuited for studies involving infants and children. However, since fNIRS does not directly provide anatomical or structural information, these measurements may be sensitive to individual or group level differences associated with variations in head size, depth of the brain from the scalp, or other anatomical factors affecting the penetration of light into the head. This information is generally not available in pediatric populations, which are often the target of study for fNIRS. Anatomical magnetic resonance imaging information from 90 school-age children (5 to 11 years old) was used to quantify the expected effect on fNIRS measures of variations in cerebral and extracerebral structure. Monte Carlo simulations of light transport in tissue were used to estimate differential and partial optical pathlengths at 690,780, 808, 830, and $850 \mathrm{~nm}$ and their variations with age, sex, and head size. This work provides look-up tables of these values and general guidance for future investigations using fNIRS sans anatomical information in this child population. ( ) The Authors. Published by SPIE under a Creative Commons Attribution 3.0 Unported License. Distribution or reproduction of this work in whole or in part requires full attribution of the original publication, including its DOI. [DOI: 10 .1117/1.NPh.5.1.011009]
\end{abstract}

Keywords: near-infrared spectroscopy; anatomical variability; structural anatomy; Monte Carlo simulation; radiative transport equation.

Paper 17064SSR received Apr. 5, 2017; accepted for publication Aug. 16, 2017; published online Sep. 18, 2017.

\section{Introduction}

Functional near-infrared spectroscopy (fNIRS) is a noninvasive neuroimaging technique that uses low levels of red to near-infrared light (650 to $950 \mathrm{~nm}$ ) to measure changes in the optical absorption of tissue due to hemoglobin. In this region of wavelengths, often referred to as the "optical window," light can propagate up to several centimeters through tissue, which is deep enough to reach parts of the cerebral cortex from optical emitters and detectors typically placed on the surface of the scalp. Using a grid of these sensors placed within a head cap and worn by the participant, the underlying changes in evoked cerebral hemodynamic responses can be spatially and temporally recorded. The precise penetration of light into the brain, however, depends on a number of individual factors such as optical scattering and the anatomical structure of the brain and extracerebral layers. Variations between individuals in the cortical folding of the brain, head-size, skull thickness, or layers of cerebral spinal fluid (CSF) can influence the recorded fNIRS signals and the sensitivity to underlying brain activity. This is a limiting situation since one of the advantages of fNIRS technology is often cited as its portability, low cost, and ability to record brain activity from pediatric or other special subject populations for whom magnetic resonance imaging

*Address all correspondence to: Theodore Huppert, E-mail: huppertt@upmc .edu
(MRI) may be difficult or contraindicated. The objective of this current work is to investigate how these factors affect fNIRS measurements in school-age children and to systematically examine the quantitative effect of age, head-size, and sex on fNIRS measurements. Specifically, we examine the effects on the optical differential pathlength factor (DPF) and partial pathlength factor (PPF) using Monte Carlo modeling of the optical transport model. A dataset of structural MRI volumes from 90 children (58 to 131 months) is examined in this work.

\subsection{Functional Near-Infrared Spectroscopy Imaging}

Over the last 40 years, since it was first demonstrated by Jobsis, ${ }^{1}$ fNIRS has been applied to a growing number of applications in psychology, psychiatry, and brain development. ${ }^{2-6}$ In particular, the application of fNIRS in child and infant populations has been successfully demonstrated by numerous researchers. ${ }^{7-12}$ Compared to functional magnetic resonance imaging (fMRI), fNIRS recordings can be made in a nonrestrictive environment and do not require a specialized scanning room nor the participant to lie in a motionless supine position. During fNIRS imaging, the participant can sit or even stand while wearing the fNIRS head cap, allowing reasonable movement of the participant and interactions with other people or the environment.

While a number of researchers have used fNIRS to examine group-level changes in brain activity with respect to subject age 
or sex in the context of child development, a persistent underlying confound of such work is the potential for systematic differences in the underlying structure of the head, brain, and other factors that affect the magnitude of the fNIRS signal. While light in the near-infrared window can penetrate up to several centimeters of tissue due to low intrinsic absorption in this range, light passing through the tissue is highly scattered resulting in the diffusion of the light through the tissue. Thus, the path of this scattering depends on the structure of the head, particularly the boundaries of the brain, skull, and CSF. In these layers, systematic differences with age or sex would result in a bias in the reported magnitude of the fNIRS recordings. For instance, in older adults, atrophy of the frontal and temporal cortices ${ }^{13}$ could result in a decrease in the sensitivity and reported magnitude of measured brain activation as the distance increases between the brain and the surface of the scalp where the fNIRS sensors are positioned. ${ }^{14}$ Similarly, in children, the growth of the head and/ or brain could cause similar biases. Previous work by Beauchamp et al. ${ }^{15}$ examined changes in the structure of the brain over a range of pediatric structural MRI volumes in 71 children ages 0 to 12 year old. While this work documented the changes in the scalp-brain distance with age, the direct quantitative impact on fNIRS was not examined. In particular, these changes would have an effect on the optical path of light in tissue and the fraction of the signal coming from the actual brain compared to the superficial layers.

\subsection{Modified Beer-Lambert Law}

Cope et al. ${ }^{16}$ introduced the concept of the modified BeerLambert law (MBLL) as a way to approximate the effect of scattering on the propagation of light in tissue and the resulting increase in the effective distance (optical pathlength) that light travels as it moves through the tissue. The modified Beer-Lambert relationship is given by

$$
\Delta \mathrm{OD}^{\lambda} \cong\left(\sum_{i} \varepsilon_{i}^{\lambda} \cdot c_{i}\right) \cdot L \cdot \mathrm{DPF}^{\lambda} \cdot \mathrm{PVF}^{\lambda}
$$

where $\Delta \mathrm{OD}$ is the change in optical density (absorption) measured between an fNIRS source-to-detector pair, $\varepsilon$ is the extinction coefficient at a particular wavelength $(\lambda)$ and for a particular $i$ 'th chromophore, and $c_{i}$ is the concentration of that chromophore. In the case of fNIRS, the two chromophores of interest are typically oxy- and deoxy-hemoglobin $\left(\mathrm{HbO}_{2}\right.$ and $\mathrm{Hb}$, respectively). In the original Beer-Lambert law, optical density is proportional to the optical pathlength through the sample. However, in the MBLL, this is replaced by an effective pathlength to account for scattering of the light and the diffuse path that photons will travel in the tissue. The effective pathlength through the tissue is approximated by the product of the distance along the surface between a source-detector pair $(L)$ and a wavelength correction term called the DPF, which is a unitless scalar that adjusts for scattering. As an example, for a scalp distance $(L)$ of $3 \mathrm{~cm}$ between an fNIRS source-detector pair, the photon will typically travel an effective distance of $\sim 18 \mathrm{~cm}$ as it scatters back and forth though the tissue. In this case, the DPF would be $6(18 \mathrm{~cm}=3 \mathrm{~cm} \times 6)$. However, of this $18 \mathrm{~cm}$, most of this is through the extracerebral skin, skull, and CSF layers that are of little interest to fNIRS. Thus, the partial volume factor (PVF) in Eq. (1) is applied to adjust for the fraction of this path that is actually in the brain. The resulting correction to the MBLL $\left(L \cdot \mathrm{DPF}^{\lambda} \cdot \mathrm{PVF}^{\lambda}\right)$ represents the effective pathlength through the brain volume of interest specifically. The PPF is defined as

$\mathrm{PPF}^{\lambda}=\mathrm{DPF}^{\lambda} \cdot \mathrm{PVF}^{\lambda}$,

giving the brain-specific MBLL as

$\Delta \mathrm{OD}^{\lambda} \cong\left(\sum_{i} \varepsilon_{i}^{\lambda} \cdot c_{i}\right) \cdot L \cdot \mathrm{PPF}^{\lambda}$.

Thus, PPF is a wavelength specific, unitless scalar that adjusts for the effective pathlength in the brain. In contrast, DPF adjusts for the pathlength through all tissues. Since the optical measurements between a source-to-detector pair $(\Delta \mathrm{OD})$ are proportional to PPF and the magnitude of the signal change in the brain, the PPF term is directly relevant in examining systemic differences with age, sex, or head size.

\section{Methods}

\subsection{Subject Population}

All MRI data were collected at 3 Tesla on a Siemens TIM TRIO scanner using T1-wieghted MPRAGE imaging. All subjects had

Table 1 Subject demographics.

\begin{tabular}{|c|c|c|c|c|c|c|c|c|}
\hline & \multicolumn{4}{|c|}{ Males $(n=46)$} & \multicolumn{4}{|c|}{ Females $(n=44)$} \\
\hline & Mean & StdDev & Min & Max & Mean & StdDev & Min & Max \\
\hline Age (months) & 96.1 & 15.9 & 60.9 & 124.2 & 92.3 & 17.7 & 58.1 & 131.4 \\
\hline Weight (Kg) & 28.9 & 7.3 & 15.9 & 46.3 & 30.6 & 10.7 & 15.9 & 68.0 \\
\hline Height (m) & 1.29 & 0.10 & 1.02 & 1.50 & 1.26 & 0.14 & 0.91 & 1.52 \\
\hline Head circumference $(\mathrm{cm})$ & 38.9 & 2.4 & 33.7 & 44.5 & 38.7 & 2.0 & 34.0 & 42.6 \\
\hline Arc length AP $(\mathrm{cm})$ & 37.1 & 1.1 & 34.5 & 39.0 & 37.3 & 1.3 & 34.5 & 40.2 \\
\hline Arc length $R L(\mathrm{~cm})$ & 34.5 & 0.9 & 32.2 & 36.1 & 34.7 & 1.2 & 32.2 & 37.4 \\
\hline
\end{tabular}

Note: 3-T MRI data from a total of 90 subjects was used in this study. Age (months), height, and weight were taken at the time of scan. Head circumference and the arc length in the anterior-posterior (AP; nasion to inion) and right-left (between preauricular points) directions were computed post hoc from the structural MRI data as described in the text. 
participated in one of the several imaging studies between 2012 and 2015 as part of the healthy/control cohort at the University of Pittsburgh and provided written IRB consent via parent/ guardian proxy. ${ }^{17}$ Structural MR images from a total of 95 subjects were used in this work. Five of the subjects were removed from analysis due to low MRI quality and/or errors in the anatomical registration or segmentation algorithms. Of the 90 remaining subjects, 46 were female. The age range was 58 to 131 months (mean 95 months; SD 17 months). The demographics of these subjects are provided in Table 1.

\subsection{Magnetic Resonance Imaging Processing and Segmentation}

The structural T1-weighted MR images were processed through the FreeSurfer-based ${ }^{18}$ HCP structural pipelines. ${ }^{19}$ This automated processing pipeline is designed to produce minimally distorted structural volumes for each subject both in "native" space and standardized Montreal Neurological Institute (MNI) space.

T1-weighted images were internally cropped to a smaller field of view to remove the neck using FSL's "robustfov" tool, then aligned to the MNI template space using a 12 degree-offreedom affine FMRIB's linear image registration tool. A brain mask was applied and then a 6-degree-of-freedom transform was used to align the anterior commissure (AC), posterior commissure (PC), and AC-PC line. The AC-PC aligned brain extracted images were then registered linearly and nonlinearly to the MNI template. These warps were inverted and the template brain masks were brought back into the AC-PC aligned space.

The aligned T1-weighted images were then intensity normalized and FreeSurfer's "recon-all" function was run to generate white matter and pial surfaces. Pial surfaces were generated using Gaussian parameters (3 standard deviations above and below gray matter mean intensity). Morphometric measurements of volumes, surface areas, and thicknesses were then computed from these surfaces.

A secondary segmentation was performed on the skin, skull, and CSF layers using the FreeSurfer watershed algorithm "mri_watershed." 20 The watershed segmentation algorithm was used to determine the intensity values for white matter, gray matter, and CSF. An elliptical surface was fitted to the brain and the shape of the surface fit was evaluated against a previously derived template. The brain surface file was then grown outward to generate an inner skull surface. A fifth-order icosahedral surface was fit around the outer edge of the volume and smoothed to make the skin surface. Finally, this skin surface was shrunk to make the outer skull surface. Following automated segmentation using the FreeSurfer and "mri_watershed" algorithms, the brain head-layer surfaces were visually examined. A total of five subjects were discarded due to either poor segmentation, registration, or general data quality leaving 90 final datasets that were used in analysis.

\subsection{Functional Near-Infrared Spectroscopy Modeling}

Each of the 90 subjects' segmented anatomical volumes was used to model the sensitivity and characteristics of theoretical fNIRS measurements using Monte Carlo simulations. ${ }^{21,22} \mathrm{~A}$ four-layer (skin, skull, CSF, and brain) anatomical meshbased model was created from the segmented boundaries of the skin, outer skull, inner skull, and pial surface of the brain using the iso2mesh program from Fang and Boas. ${ }^{23}$ Monte Carlo simulations were run at five wavelengths $(690,780$,
Table 2 Tissue optical properties for Monte Carlo simulations.

\begin{tabular}{|c|c|c|c|c|c|c|}
\hline & & \multicolumn{5}{|c|}{ Wavelength $(\mathrm{nm})$} \\
\hline & & 690 & 780 & 808 & 830 & 850 \\
\hline \multirow[t]{4}{*}{ Skin } & $\mu_{A}\left(\mathrm{~mm}^{-1}\right)$ & 0.021 & 0.014 & 0.012 & 0.012 & 0.012 \\
\hline & $\mu_{S}^{\prime}\left(\mathrm{mm}^{-1}\right)$ & 2.91 & 2.45 & 2.33 & 2.24 & 2.16 \\
\hline & $N$ & 1.45 & 1.45 & 1.45 & 1.45 & 1.45 \\
\hline & $g$ & 0.89 & 0.89 & 0.89 & 0.89 & 0.89 \\
\hline \multirow[t]{4}{*}{ Skull } & $\mu_{A}\left(\mathrm{~mm}^{-1}\right)$ & 0.026 & 0.025 & 0.025 & 0.025 & 0.027 \\
\hline & $\mu_{S}^{\prime}\left(\mathrm{mm}^{-1}\right)$ & 1.82 & 1.67 & 1.62 & 1.59 & 1.57 \\
\hline & $N$ & 1.45 & 1.45 & 1.45 & 1.45 & 1.45 \\
\hline & $g$ & 0.89 & 0.89 & 0.89 & 0.89 & 0.89 \\
\hline \multirow[t]{4}{*}{ CSF } & $\mu_{A}\left(\mathrm{~mm}^{-1}\right)$ & 0.001 & 0.002 & 0.002 & 0.003 & 0.004 \\
\hline & $\mu_{S}^{\prime}\left(\mathrm{mm}^{-1}\right)$ & 0.01 & 0.01 & 0.01 & 0.01 & 0.01 \\
\hline & $N$ & 1.33 & 1.33 & 1.33 & 1.33 & 1.33 \\
\hline & $g$ & 0.89 & 0.89 & 0.89 & 0.89 & 0.89 \\
\hline \multirow{4}{*}{$\begin{array}{l}\text { Brain } \\
\text { (gray + } \\
\text { white) }\end{array}$} & $\mu_{A}\left(\mathrm{~mm}^{-1}\right)$ & 0.010 & 0.011 & 0.011 & 0.012 & 0.014 \\
\hline & $\mu_{S}^{\prime}\left(\mathrm{mm}^{-1}\right)$ & 1.44 & 1.18 & 1.12 & 1.07 & 1.03 \\
\hline & $N$ & 1.45 & 1.45 & 1.45 & 1.45 & 1.45 \\
\hline & $g$ & 0.89 & 0.89 & 0.89 & 0.89 & 0.89 \\
\hline
\end{tabular}

Note: This table provides the optical properties for the skin, skull (bone), CSF, and brain layers used in the Monte Carlo simulations. Optical properties were adopted from Jacques. ${ }^{19} \mu_{A}$ is the optical absorption coefficient, $\mu_{s}^{\prime}$ is the reduced scattering coefficient, $N$ is the index of refraction, and $g$ is the anisotropy coefficient.

808,830 , and $850 \mathrm{~nm}$ ). The optical properties for these tissues and wavelengths are given in Table 2 . In this work, gray and white matter brain tissues were assigned the same optical properties similar to the earlier work in Strangman et. al. ${ }^{24}$

The optical properties were computed from the general tissue models given by Jacques ${ }^{25}$ and optical scattering $\left(\mu_{s}^{\prime}\right)$ and absorption $\left(\mu_{A}\right)$ was computed by

$$
\begin{aligned}
\mu_{s}^{\prime}= & a \cdot\left[\frac{\lambda}{500(\mathrm{~nm})}\right]^{-b} \\
\mu_{A}= & B \cdot S \cdot \mu_{A, \mathrm{HbO} 2}+B \cdot(1-S) \cdot \mu_{A, \mathrm{Hb}} \\
& +W \cdot \mu_{A, \text { water }}+F \cdot \mu_{A, \mathrm{fat}},
\end{aligned}
$$

where the parameters for these two equations were compiled from various empirical sources in the literature as given in Jacques. ${ }^{25}$ For scattering, the parameters for the skin $\left(a=4.6 \mathrm{~mm}^{-1}, b=\right.$ $1.421)$, bone $\left(a=2.29 \mathrm{~mm}^{-1}, b=0.716\right)$, and brain $(a=$ $\left.2.42 \mathrm{~mm}^{-1}, b=1.611\right)$ were used. For the brain, we assumed oxygen saturation $(S)=70 \%$, blood volume fraction $(B)=$ $2.2 \%[=50 \mu \mathrm{M} \times(66,458 \mathrm{gm} / \mathrm{mol}) /(150 \mathrm{gm} \mathrm{Hb} / \mathrm{L}$ blood $)]$, water fraction $(W)=70 \%$, and fat fraction $(F)=0 \%$. For the other tissues, we used values given by Jacques. ${ }^{25}$ 
(a)

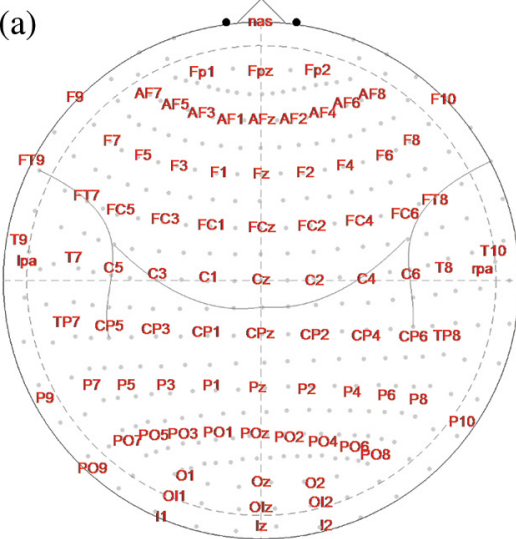

(b)

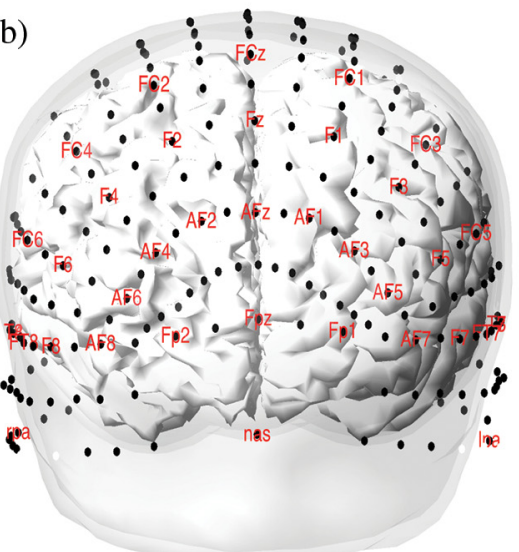

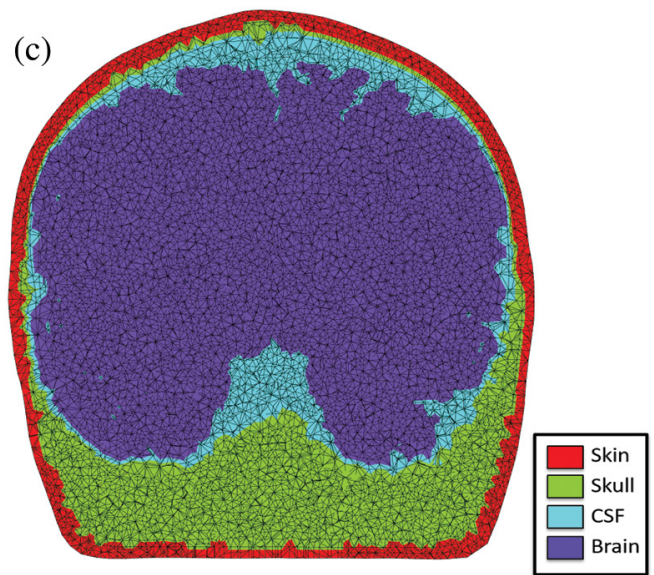

Fig. 1 Example of segmentation and Monte Carlo simulations. (a) and (b) The locations of the 10-5 coordinate points on the surface of one of the subjects and the equivalent polar projection of this map. (c) An example mesh used for the Monte Carlo simulations in the same subject.

For each subject, we modeled the optical sensitivity (forward model) using a mesh-based Monte Carlo method. ${ }^{22}$ Functional NIRS sources were modeled from 346 positions on the head for each subject at the international 10-5 coordinate positions (see Fig. 1). For each source and wavelength, $5 \times 10^{7}$ photons were simulated. Thus, over the whole cohort, 157,000 simulations were run for a total of 72,000 CPU-hours of computing on a $240 \mathrm{CPU}$ high-performance computing cluster. For each simulation, the exiting photons were monitored at all other positions on the surface of the head yielding a $346 \times 2564$ matrix of virtual fNIRS source-detector combinations from around each source position.

For each simulated source-detector pair, the optical pathlength through the entire tissue (DPF) and partial pathlength through the brain (PPF) was recorded. The DPF and PPF were computed for each 10-5 position by fitting the $\sim 120$ measurements between 10 and $40 \mathrm{~mm}$ around each source to a linear regression model using an iterative robust regression algorithm (MATLAB function "robustfit").

\subsection{Topographic Projections}

Each subject was registered to a $10-5$ head coordinate space by aligning the left/right preauricular ear points and nasion point in the MRI data to an elliptical template of 10-5 points included in the SPM software ("ext1020.sfp") $)^{26}$ followed by an iterative closest point registration and refinement using the head surface. Based on this head coordinate registration, the head circumference (at $10 \%$ up along the arc length between preauricular ear points) and the arc lengths from nasion to inion and between preauricular ear points was computed retrospectively.

To compare estimated parameters (cortical depth, optical pathlength, etc.) across subjects, values of interest were first projected along the surface normal to the nearest position on the scalp. The scalp positions were then projected using a Clarke's twilight azimuthal projection using a normalized head radius into a two-dimensional topographic map and interpolated onto an equidistant polar grid. This projection allowed data from subjects to be compared in a standardized space independent of the subject head circumference and size. Except where noted, the median value of the parameters was computed across subjects in this polar (10-5 coordinate) space.

\section{Results}

\subsection{Characterization of Anatomical Variations}

Using the anatomical MRI volumes from the 90 children, we computed the median skull thickness and CSF layer thickness according the 10-5 space projected values. The median value for the male and female groups is shown in Fig. 2. The skull thickness ranged between 1.1 and $9.6 \mathrm{~mm}\{2.51 \pm 0.92$ $[$ median $\pm 0.67499 \times($ median absolute deviation $)]\}$ in the females and 1.1 to $8.3 \mathrm{~mm}(2.66 \pm 0.92)$ in the males. In both sexes, the skull thickness at the crown and posterior of the head (posterior of 10-5 position FpZ and lateral out to positions $\mathrm{Cp} 3 / \mathrm{Cp} 4$ ) was about 2 to 3 times thicker ( 3.2 to $5 \mathrm{~mm}$ ) than the other frontal or temporal regions as shown in Fig. 2.

The CSF thickness (defined as the distance between the inner surface of the skull and the pial surface of the brain) was

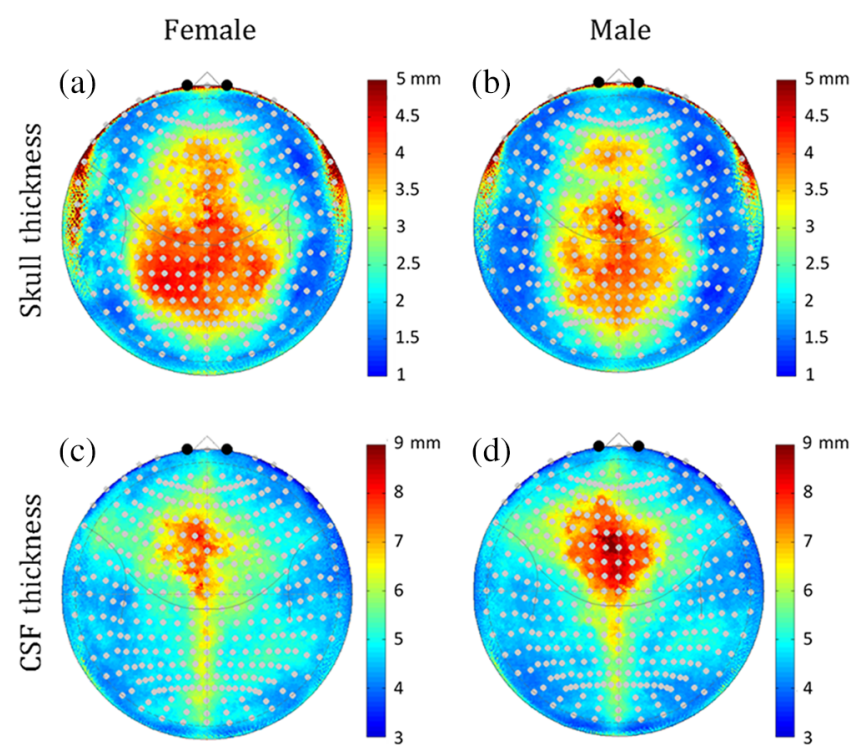

Fig. 2 Gender differences in skull CSF layer thickness. (a) and (b) The median skull and (c) and (d) CSF thickness for the male and female subjects is shown in the normalized polar (10-5 coordinate) space. The color scales show the thickness of these layers in millimeters. 
between 3 and $9 \mathrm{~mm}$ in both sexes (male 5.12 \pm 0.95 ; female $5.05 \pm 0.89$ ) and greatest ( 7 to $9 \mathrm{~mm}$ ) at the slightly anterior to the crown of the head around 10-5 position $\mathrm{Fz}$ and extending posterior along the sagittal sulcus. The area of this thicker CSF region was larger in the males than the females. In our segmentations, we did not consider the venous dural sinuses, and some of this CSF thickness at the sagittal sulcus is likely due to the sagittal sinus, which is labeled as CSF in the segmentations.

The depth of the surface of the cortex relative to the scalp is shown in Figs. 3(a) and 3(b) for both sexes. The cortical depth ranged from 6.2 to $14.7 \mathrm{~mm}$ in females (median $10.2 \pm 1.8$ ) and 6.6 to $14.5 \mathrm{~mm}$ in males (median $10.13 \pm 1.73$ ). This was lowest $(7$ to $9 \mathrm{~mm}$ ) along the frontal cortex and bilateral lateral regions. This depth was about 50\% deeper along the top of the head down the midline where it ranged from about 11 to $15 \mathrm{~mm}$. This pattern reflected the same regions that had been observed to have thicker skull or CSF layers shown in Fig. 2 for the two sexes. Figure 3(c) shows the difference map of the cortical depth of the females verses the males. Regions in red color show parts of the cortex that were deeper in the females compared to the males. On average, the cortex was $0.1 \mathrm{~mm}$ (range $[-5.6$ to 4.89$] ; \quad p<1 \times 10^{-15} ; F_{30420,1}$. two-way ANOVA with position and group) deeper in the female participants. Notably, the medial and right frontal/medial cortex was between 2 and $3.5 \mathrm{~mm}$ deeper in the females compared to the males. The deeper depth of the cortex in the females means that fNIRS measures will be less sensitive to changes in brain activity in the females compared to the males. This could result in underestimation of brain activity in the female group from these regions and a sex-related bias in the activity in group-level analysis models.

We also examined variability in the location of the cortical folding pattern (mapped according to MNI space) relative to the 10-5 head coordinate system. Using FreeSurfer, the cortical surfaces are extracted and registered as surfaces into the "fsaverage" space where parcellation labels are assigned. In this space, the equivalent anatomical regions can be morphed from one subject's anatomy onto another. Figure 4 shows the median displacement in the equivalent fsaverage cortical positions relative to the surface of the head in both the males and females. The most conserved anatomical regions between subjects [shown in blue-green colors in Figs. 4(a) and 4(b)] were in the lateral frontal, temporal, and posterior/occipital regions and varied from about 5 to $30 \mathrm{~mm}$ median displacements. The most anatomically variable regions were along the midline and superior frontal regions of the head which varied up to 20 to $30 \mathrm{~mm}$. Specifically, these regions of high variability in the underlying
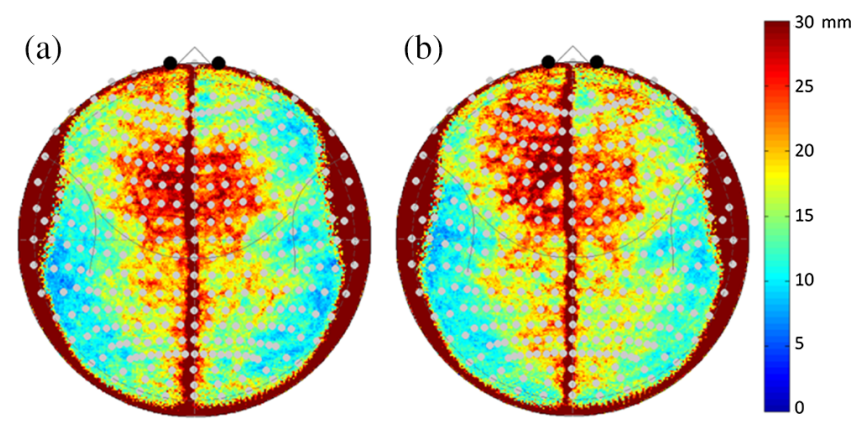

Fig. 4 Underlying variability in brain (MNI) space measured from scalp-based fNIRS sensors. This figure shows the root-mediansquared displacement in underlying registered $\mathrm{MNI}$ coordinates of the surface of the brain as measured from each position on the scalp. Red color indicates higher variability in the underlying structure and folding of the brain beneath each position and indicates areas where fNIRS measurements would have lower precision due to individual differences in the folding of the cortex. The results for the (a) female and (b) male subjects are shown with the same color scale.

structure of the cortical folding are the regions where the alignment of fNIRS measurements according to the $10-5$ positioning on the scalp would be more sensitive to unknown variability in the underlying brain region.

Finally, in Fig. 5, we looked at the effect of head size and age on the cortical depth. The registered polar (10-5 coordinate) space data for all 90 subjects across both sexes was pooled and regressed using the head circumference or subject age in a robust regression model. In Fig. 5, we show the $t$-statistic map for these two models. As shown in Fig. 5(a), we found that head circumference was more correlated to the depth of the brain than age. This is because over our group of subjects in this school-age range, we found that there was a considerable spread in head size, height, and weight, which was not well predicted by age alone and reflected different growth rates for the children. Specifically, head circumference was only marginally related to age $\left(R^{2}=0.176\right)$. We found that the depth of the brain at the top of the head was positively related to head circumference [Fig. 5(a)], which indicates that this depth was greater for children with larger head sizes. This also indicates that the head is probably growing faster than the brain over this age range resulting in an increasing depth.

\subsection{Variation in Optical Properties}

As previously described in the methods, a Monte Carlo-based model was used to simulate fNIRS measurements for each of (a) Female

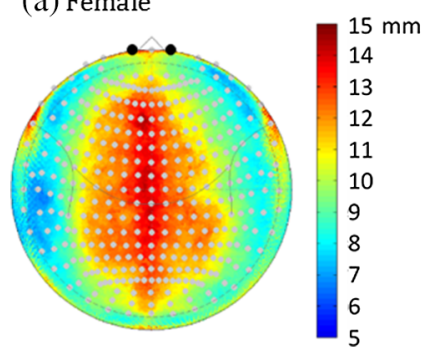

(b) Male

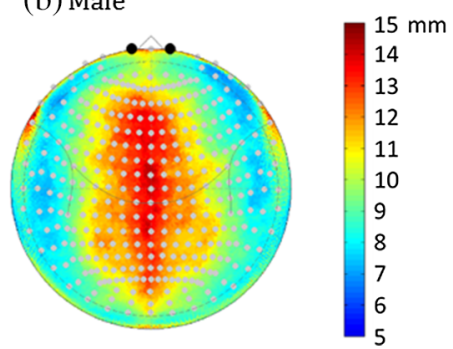

(c) Female - Male

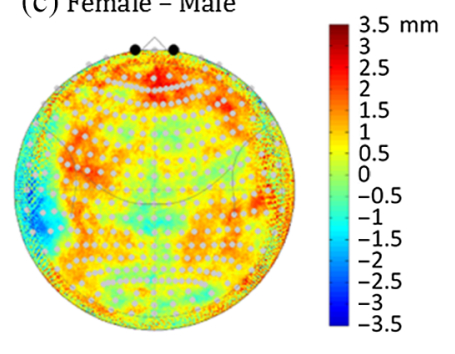

Fig. 3 Gender differences in the scalp-to-brain depth. (a) and (b) The median distance normal to the surface from the scalp to the nearest position on the pial cortical surface in the subject normalized polar (10-5 coordinate) space. (c) The difference between the depth of the females compared to the males. In most regions, the brain of the female subjects up to 3-mm deeper. 

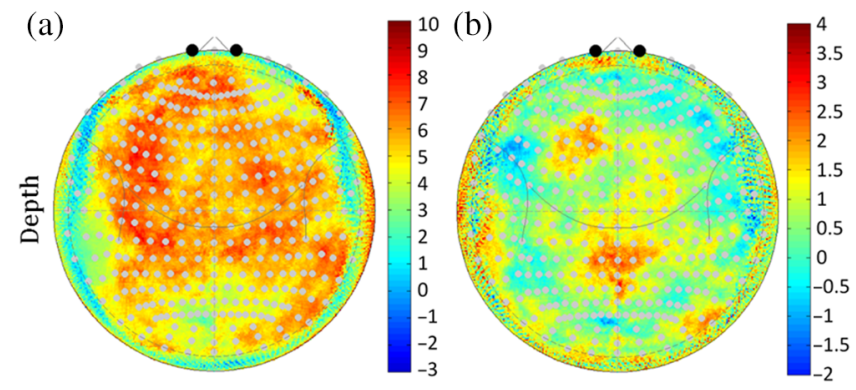

Fig. 5 Correlation of scalp-to-brain depth with head circumference and age. This figure shows $t$-statistic maps (89 degrees of freedom) from the pooled data across both genders for a regression model against (a) head circumference and (b) age at time of MRI scan in months. Red indicates a positive correlation and increasing depth with circumference or age.

the 90 subjects at all 346 of the $10-5$ positions on the head for wavelengths $690,780,808,830$, and $850 \mathrm{~nm}$. These wavelengths were selected as the five most widely used wavelengths in currently commercially available fNIRS systems. At each 105 head position, virtual "photons" from an fNIRS emitter were simulated and mapped to all surface points symmetrically around the emitter. For each emitter position, the DPF was computed by a weighted least-squares fit of the detectors from 10 to $40 \mathrm{~mm}$ around a source. The differential pathlength (e.g., how far did the light travel within the tissue) is equal to the product of the DPF and the emitter-detector distance along the arc of the scalp [Eq. (1)]. Similarly, the PPF as defined in Eq. (2) was computed as the pathlength through only the cortex layer of the model. Both DPF and PPF are unitless scaling factors, which adjust the fNIRS emitter-detector distance in the MBLL.

Figure 6 shows the topographic maps of the estimated DPF for both sexes at all five simulated wavelengths. The DPF was highest for the $690 \mathrm{~nm}$ wavelength in both sexes; DPF ranged from 5.53 to 6.82 in the females (median $=6.00$ ) and from 5.38 to 6.84 in the males (median $=6.04)$. Although lower in comparison, in all other wavelengths $(780,808,830$, and $850 \mathrm{~nm})$, the median DPF between sexes was similar; 5.76, 5.71, 5.67, and 5.64 in females and 5.84, 5.79, 5.76, and 5.74 in the males, respectively). The DPF was slightly higher in the males by $1.3 \%$ to $1.6 \%$ compared to the females. Spatially, the DPF was symmetric across hemispheres and was highest along the top of the head along precentral sulcus and superior frontal cortex. The average DPF for several head regions is given in Table 3. Tables 5-15 for the DPF for both sexes and at each of the 346 10-5 coordinate positions are given in the Appendix.

Figure 7 shows the spatial distribution of the PPF for the two sexes and five simulated wavelengths. The PPF has less variation between wavelengths compared to DPF, but has a larger difference between the two sexes. The PPF was higher in the males by $15.4 \%$ to $18.8 \%$ across the whole head when compared to the females. The whole head median value of the PPF was $1.77,1.77,1.77,1.80$, and 1.90 for the females and 2.45, $2.44,2.48,2.45$, and 2.45 for the males at $690,780,808$, 830 , and $850 \mathrm{~nm}$, respectively. Although this difference was observed across the whole head, PPF sex differences were the greatest in the area around the medial frontal region (Fp1, FpZ, and Fp2); here females had a lower PPF value (1.15 compared to 1.45 [average of all 5 wavelengths; $\left.p=1.5 \times 10^{-4}\right]$ ). This corresponds to the same region around the frontal sinuses where the cortex was deeper among females, as shown in Fig. 3(c). The observed 2 to $3 \mathrm{~mm}$ increased depth in the females and corresponding decrease in the PPF directly translates to an expected $26 \%$ underestimation in fNIRS measurements of brain activity in these regions relative to males. The PPF across the head is summarized in Table 3. Due to space constraints, both males and females have been combined in Table 3. However, for both sexes, Tables 5-15 describing the 346 10-5 coordinate positions are given in the Appendix.

Similar to the analysis of the scalp-to-brain depth with age and head circumference, we also examined DPF and PPF with these two regression models. Figure 8 shows the statistical result ( $t$-statistic) from the regression model pooling data from all 90 subjects. Both head circumference and age had the biggest effect on the DPF in the frontal and occipital regions of the head with positive correction (e.g., increasing DPF with increased age or head circumference). For PPF, age was positively correlated with PPF in the frontal regions. Head circumference seemed to matter only along the equator of the head, but decreased

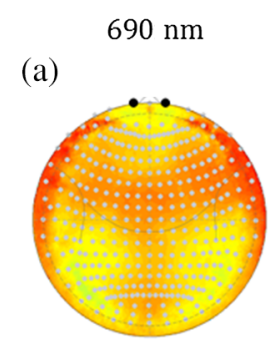

(f)

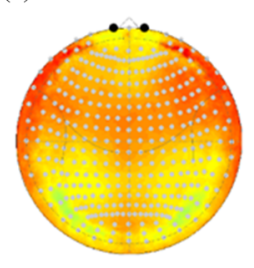

$780 \mathrm{~nm}$

(b)

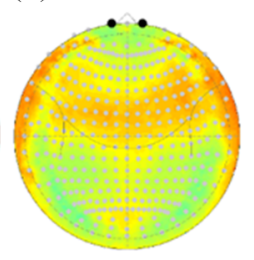

(g)

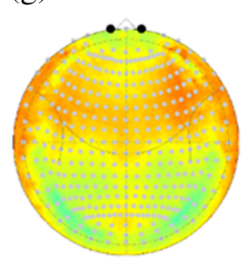

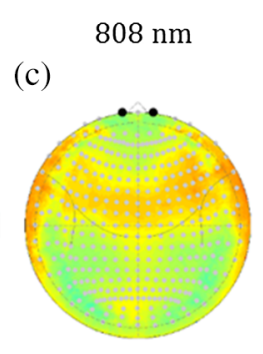

(h)

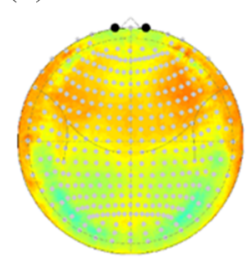

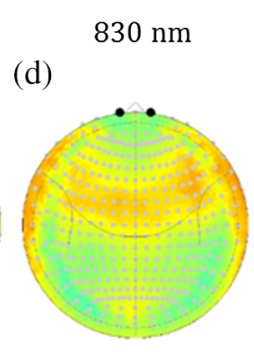

(i)

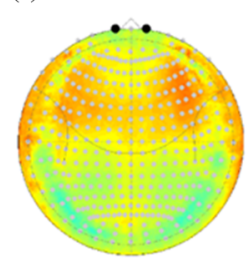

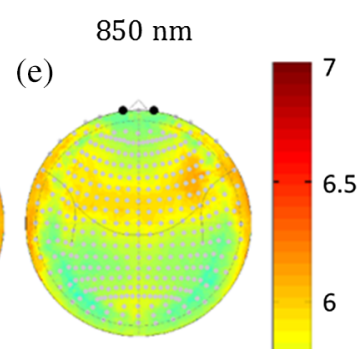

(j)

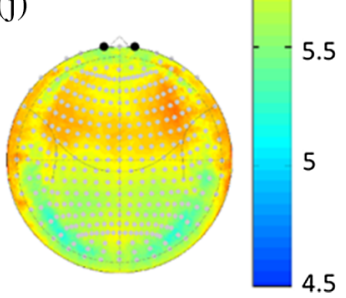

Fig. 6 Gender and wavelength differences in the DPF. The DPF at the 5 simulated wavelengths is shown for the (a)-(e) female and (f)-(j) male subjects based on the Monte Carlo simulations. The median DPF for each position in the registered polar (10-5 coordinate) space is shown for each of the wavelengths. 
Table 3 Sensitivity, DPF, PPF of anatomical regions (both sexes).

\begin{tabular}{|c|c|c|c|c|c|c|c|c|c|c|c|}
\hline \multirow[b]{2}{*}{ Region } & \multirow[b]{2}{*}{ Sensitivity (dB) } & \multicolumn{5}{|c|}{ DPF (wavelength, nm) } & \multicolumn{5}{|c|}{ PPF (wavelength, nm) } \\
\hline & & 690 & 780 & 808 & 830 & 850 & 690 & 780 & 808 & 830 & 850 \\
\hline \multicolumn{12}{|l|}{ Left hemisphere } \\
\hline Caudal middle frontal & -36.9 & 6.15 & 5.99 & 5.94 & 5.93 & 5.94 & 2.53 & 2.63 & 2.66 & 2.65 & 2.69 \\
\hline Frontal pole & -39.1 & 6.00 & 5.74 & 5.74 & 5.67 & 5.63 & 1.74 & 1.78 & 1.78 & 1.80 & 1.83 \\
\hline Inferior parietal & -29.7 & 5.98 & 5.75 & 5.73 & 5.70 & 5.66 & 2.47 & 2.43 & 2.45 & 2.50 & 2.54 \\
\hline Inferior temporal & -35.2 & 6.03 & 5.86 & 5.79 & 5.77 & 5.74 & 2.38 & 2.43 & 2.42 & 2.43 & 2.48 \\
\hline Lateral occipital & -27.2 & 6.03 & 5.75 & 5.70 & 5.66 & 5.63 & 2.28 & 2.26 & 2.27 & 2.29 & 2.35 \\
\hline Middle temporal & -28.7 & 6.05 & 5.87 & 5.82 & 5.82 & 5.77 & 2.49 & 2.54 & 2.54 & 2.54 & 2.54 \\
\hline Pars orbitalis & -41.1 & 6.10 & 5.93 & 5.92 & 5.92 & 5.88 & 2.39 & 2.44 & 2.47 & 2.48 & 2.49 \\
\hline Pars triangularis & -37.9 & 6.14 & 5.98 & 5.93 & 5.90 & 5.88 & 2.57 & 2.59 & 2.64 & 2.58 & 2.65 \\
\hline Postcentral & -30.7 & 6.13 & 5.94 & 5.91 & 5.88 & 5.87 & 2.63 & 2.70 & 2.71 & 2.72 & 2.76 \\
\hline Precentral & -33.5 & 6.13 & 6.00 & 5.95 & 5.93 & 5.92 & 2.66 & 2.72 & 2.76 & 2.75 & 2.78 \\
\hline Rostral middle frontal & -31.4 & 6.11 & 5.94 & 5.90 & 5.87 & 5.84 & 2.35 & 2.39 & 2.39 & 2.39 & 2.42 \\
\hline Superior frontal & -30.9 & 6.17 & 6.00 & 5.95 & 5.91 & 5.89 & 2.32 & 2.35 & 2.38 & 2.39 & 2.42 \\
\hline Superior parietal & -36.7 & 6.01 & 5.79 & 5.77 & 5.74 & 5.71 & 2.42 & 2.49 & 2.52 & 2.49 & 2.56 \\
\hline Superior temporal & -41.1 & 6.10 & 5.92 & 5.87 & 5.85 & 5.83 & 2.66 & 2.64 & 2.64 & 2.57 & 2.60 \\
\hline Supramarginal & -33.7 & 6.02 & 5.81 & 5.76 & 5.77 & 5.73 & 2.56 & 2.60 & 2.60 & 2.67 & 2.67 \\
\hline \multicolumn{12}{|l|}{ Right hemisphere } \\
\hline Caudal middle frontal & -38.2 & 6.22 & 6.04 & 5.97 & 5.94 & 5.91 & 1.96 & 1.98 & 2.00 & 2.04 & 2.03 \\
\hline Frontal pole & -40.3 & 6.20 & 5.93 & 5.82 & 5.81 & 5.75 & 1.38 & 1.37 & 1.38 & 1.38 & 1.39 \\
\hline Inferior parietal & -31.5 & 5.96 & 5.73 & 5.66 & 5.66 & 5.59 & 1.93 & 1.97 & 1.95 & 2.03 & 2.06 \\
\hline Inferior temporal & -35.2 & 6.02 & 5.73 & 5.67 & 5.67 & 5.61 & 1.74 & 1.72 & 1.77 & 1.70 & 1.83 \\
\hline Lateral occipital & -28.7 & 5.95 & 5.68 & 5.62 & 5.56 & 5.54 & 1.73 & 1.75 & 1.74 & 1.77 & 1.79 \\
\hline Middle temporal & -32.4 & 6.02 & 5.78 & 5.71 & 5.69 & 5.66 & 1.70 & 1.71 & 1.72 & 1.73 & 1.75 \\
\hline Pars orbitalis & -39.5 & 6.33 & 6.00 & 5.97 & 5.93 & 5.89 & 1.33 & 1.35 & 1.34 & 1.39 & 1.40 \\
\hline Pars triangularis & -35.8 & 6.28 & 6.07 & 6.05 & 6.02 & 5.95 & 1.83 & 1.81 & 1.82 & 1.86 & 1.94 \\
\hline Postcentral & -29.6 & 6.08 & 5.89 & 5.85 & 5.80 & 5.78 & 1.78 & 1.87 & 1.87 & 1.89 & 1.98 \\
\hline Precentral & -32.0 & 6.12 & 5.92 & 5.87 & 5.83 & 5.79 & 1.82 & 1.90 & 1.89 & 1.92 & 1.96 \\
\hline Rostra middle frontal & -32.8 & 6.30 & 6.05 & 5.97 & 5.94 & 5.87 & 1.60 & 1.60 & 1.64 & 1.65 & 1.64 \\
\hline Superior frontal & -34.2 & 6.17 & 6.01 & 5.95 & 5.92 & 5.89 & 1.86 & 1.92 & 1.92 & 1.93 & 1.99 \\
\hline Superior parietal & -34.9 & 6.04 & 5.78 & 5.72 & 5.68 & 5.67 & 1.94 & 1.96 & 1.96 & 1.95 & 2.02 \\
\hline Superior temporal & -38.3 & 6.06 & 5.85 & 5.81 & 5.77 & 5.74 & 1.66 & 1.65 & 1.67 & 1.68 & 1.71 \\
\hline Supramarginal & -32.2 & 6.03 & 5.84 & 5.77 & 5.74 & 5.68 & 1.74 & 1.78 & 1.82 & 1.87 & 1.93 \\
\hline
\end{tabular}

Note: This table shows the DPF and PPF for several fNIRS-accessible regions on the head. Data has been combined for both male and female subjects. The sensitivity of the region to fNIRS is provided in decibels $(\mathrm{dB} ; 10 \times \log 10)$ and indicates the fraction of photons normalized to the incidence power reaching this region from the scalp at a source-detector distance of $30 \mathrm{~mm}$. 


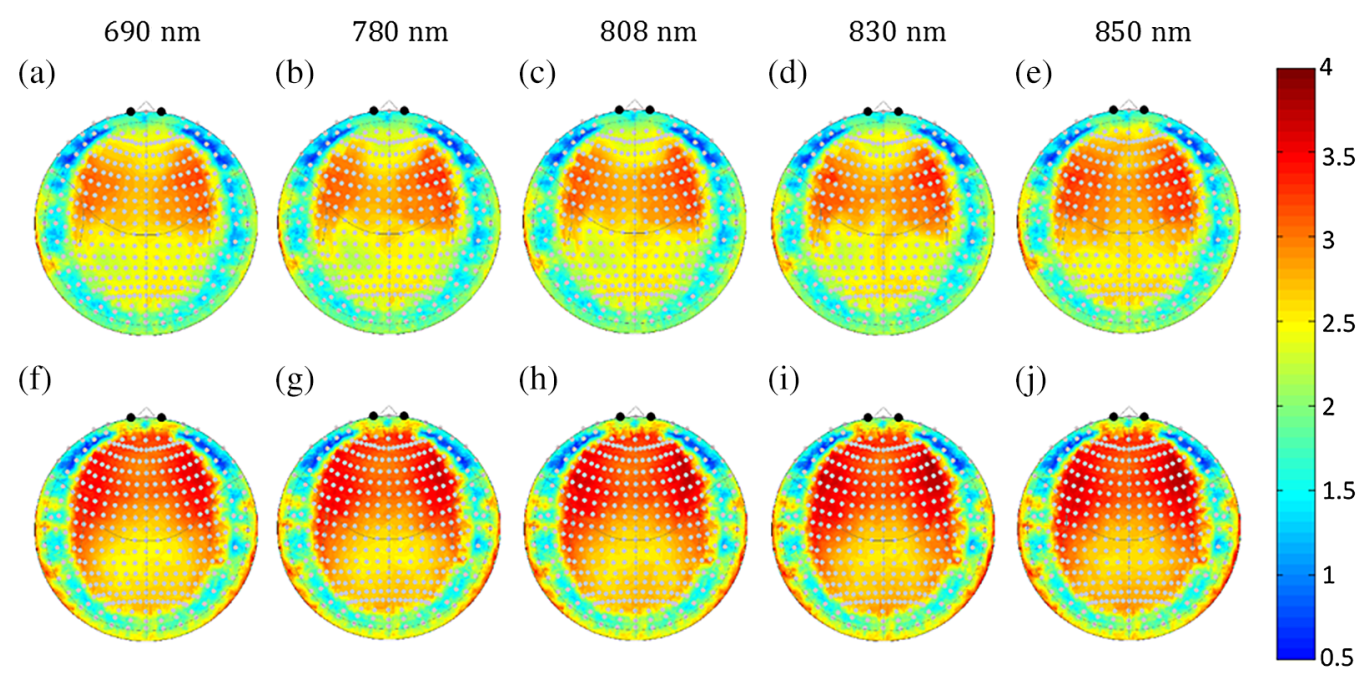

Fig. 7 Gender and wavelength differences in the PPF. The PPF at the 5 simulated wavelengths is shown for the (a)-(e) female and (f)-(j) male subjects based on the Monte Carlo simulations. The median PPF for each position in the registered polar (10-5 coordinate) space is shown for each of the wavelengths.

with head circumference in most other regions. In Figure 8, variability in DPF and PPF, seen as a noticeable ring near the equator of the Clarke projection, is due to the inferior surface of the brain cutting in where it rests on the cranium floor. This is also evident in the positional variability maps shown in Fig. 4.

\subsection{Regions-of-Interest}

The segmented MRI volumes for each of the 90 subjects were parcellated into 70 anatomical regions of the cortical surface using FreeSurfer. ${ }^{27}$ These parcellation labels were created for each subject and then projected into the registered polar (10-
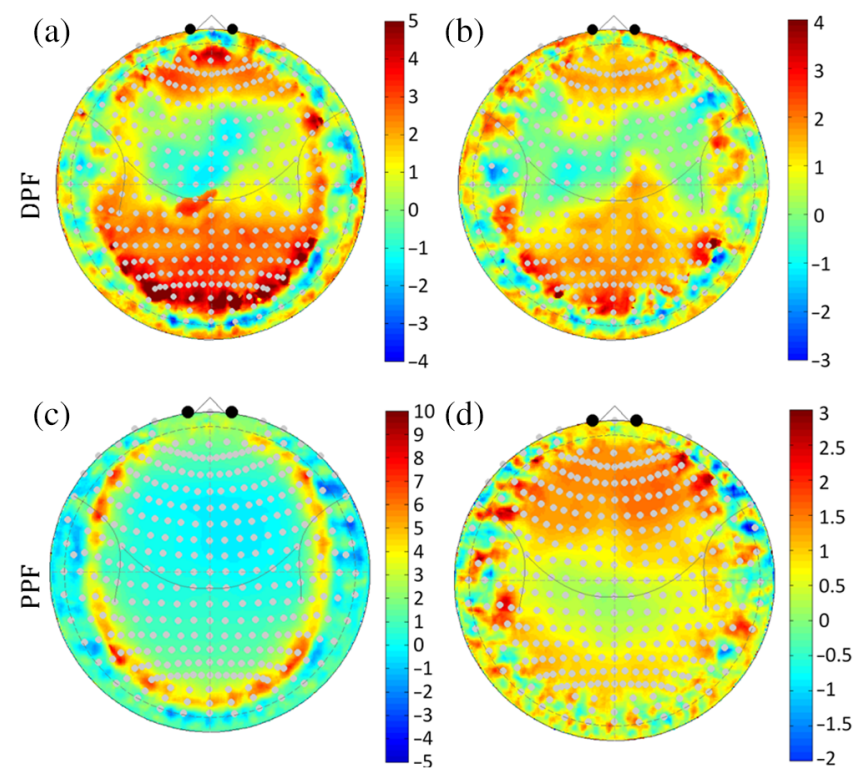

Fig. 8 Correlation of DPF and PPF with head circumference and age. This figure shows $t$-statistic maps (89 degrees of freedom) from the pooled data across both genders for a regression model against (a) and (c) head circumference and (b) and (d) age at time of MRI scan in months. Red indicates a positive correlation and increasing pathlength with circumference or age.
5 coordinate) space. Figure 9(a) shows the most frequent (mode) label across the head from all the subjects. Male and female subjects had only minor differences (see the Appendix). Similarly, we used the automatic anatomical labeling datase ${ }^{28}$ to find Brodmann area labels on the cortical surface in MNI space [Fig. 9(b)]. As a note, the Brodmann areas from the automatic anatomical labeling dataset are defined in threedimensional space and cover parts of the sulci as well as the gyri folds. Therefore, these regions extend to slightly deeper areas compared to the FreeSurfer gyri labels. In Table 4, we provide a list of the cortical depth and closest 10-5 head coordinate points for a subset of the most accessible of these regions. Tables 5-15 are provided in the Appendix.

\section{Discussion}

In this paper, we used 90 segmented MRI volumes from children ages 5 to 11 years to model the intersubject variations in head and brain anatomy and their effects on the sensitivity of fNIRS measurements. The overall objective of this paper was to quantitatively examine how variations in anatomy with age, sex, and head-size affected the optical properties of the DPF and PPF. These two factors are used in the MBLL [Eqs. (1)-(3)] to correct for the effective pathlength of light in the total tissue and brain, respectively, which in turn determines the quantitative accuracy of fNIRS. In most cases using continuous wave (CW) fNIRS recordings, the absolute quantification of the signals is not a concern. However, the potential that DPF and/or PPF could vary across spatial regions or subject demographics creates a confound that could bias group-level statistical comparisons. Namely, when DPF or PPF is ignored as a scaling factor in CW-fNIRS recordings, this assumes that these terms are constant over space/channels and subjects. This term appears in both the numerator and denominator of a $t$-test used in accessing brain activity from a linear model. ${ }^{29}$ This term would cancel only in the case of a first level model (a statistical test within a single subject and per optical channel), but not for comparing the magnitudes across channels in region-of-interest or grouplevel models. As an example from our results, although the exact value of PPF may be less of a concern, the finding that the PPF in the frontal cortex varied by about $20 \%$ in the frontal cortex 

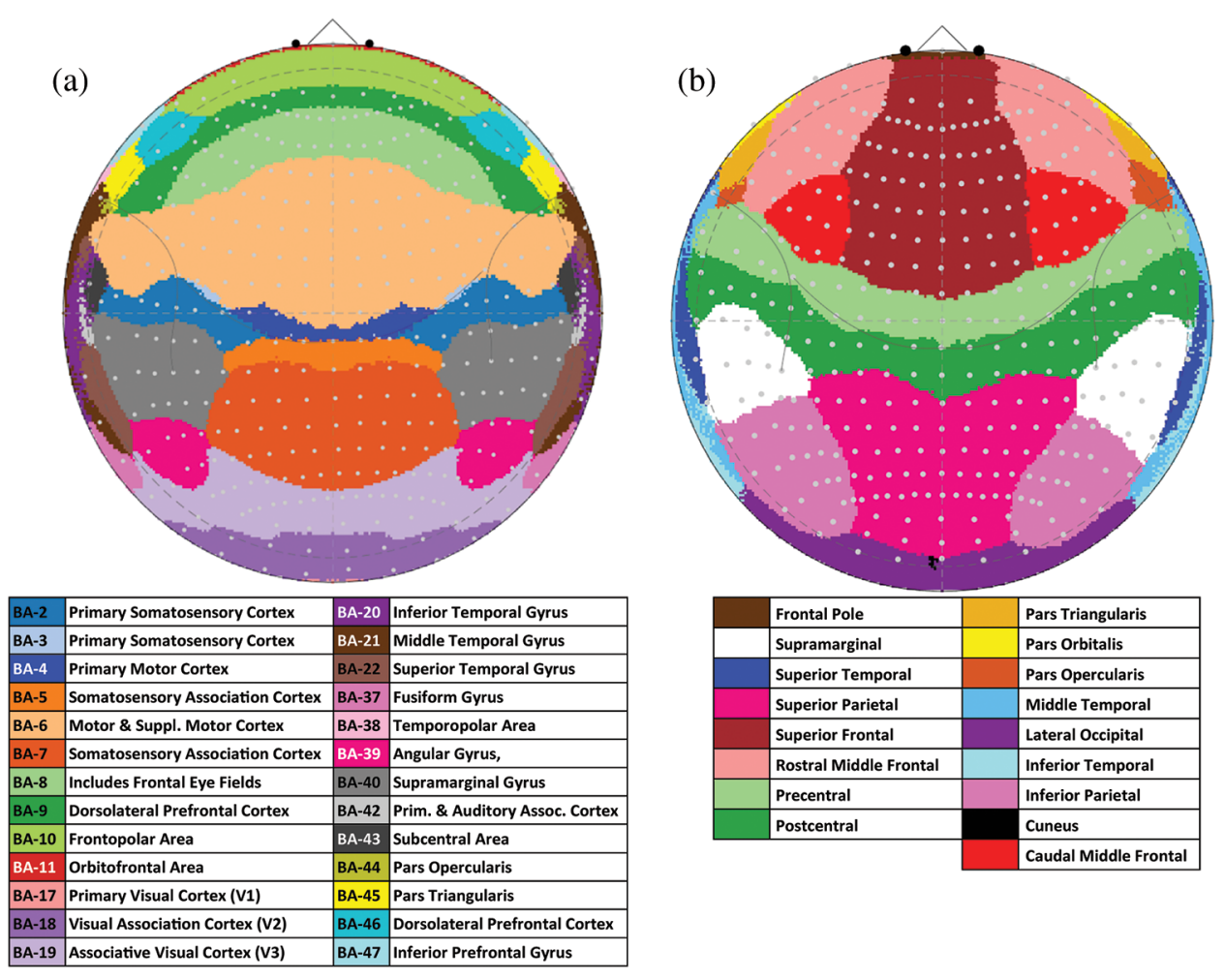

Fig. 9 Underlying anatomical and Brodmann area maps. (a) The mode label across the 90 subjects is given for the anatomical labels of the gyri from FreeSurfer. ${ }^{20}(\mathrm{~b})$ The Broadmann area labels based on the aal template ${ }^{21}$ are given. Both panels show the most frequent label (mode) across both genders.

between males and females means that there would be an expected bias toward underestimation of the magnitude of the brain signals specifically in the female group. Thus, if the magnitude of the hemodynamic response was exactly the same in the brain/cortex space of the two groups, the resulting optical measurements in the female group would be $20 \%$ smaller in these regions. While we caution against interpreting the results of statistical tests performed in fNIRS channel space as related to true differences in brain activity compared to systematic variations in anatomy, we believe that this work can provide valuable guidance toward how these biases may be addressed at the group level. Specifically, we suggest careful examination of sex difference in fNIRS datasets in future research projects.

\subsection{Anatomical Variations in Subjects}

In the first part of this paper, we examined differences in the structural anatomy of the brain with sex, head-size, and subject age based on segmentation and registration of the anatomical MRI data. In both the male and female populations, we found quite a bit of spatial variation in the depth of the brain relative to the scalp surface. While skull and CSF thicknesses were comparable across the two groups, we found that on average the cortical depth was slightly deeper in the female subjects. This difference was greatest in the frontal regions where we believe a larger sinus cavity in the female subjects is responsible for the 2 to $3 \mathrm{~mm}$ increase depth of the brain in these regions. As the distance between the brain and the scalp increases, the fraction of light reaching these brain regions decreases, therefore, we observed a lower PPF value in these regions for the female subjects. This is particularly relevant for fNIRS measurements that target these frontal regions due to accessibility of the forehead, absence of hair, and scientific interest in the underlying cognitive functional domains (e.g., Brodmann areas 10, 46, and 44). We also found an asymmetry in the cortical depth, with the right side being slightly deeper than the left side of the frontal lobe. This is consistent with the structural asymmetries noted previously in a similar analysis by Beuachamp et al. ${ }^{15}$ When we looked at head size and age, we found that head size was the better predictor of underlying anatomy rather than age in this range of 5- to 11-year-old children. Thus, a recommendation to future fNIRS studies might be to report head sizes as well as age when listing subject demographics.

In Fig. 4, we also examined variations in the cortical folding of the brain with respect to the scalp-based 10-5 mapping. We found that certain regions, including the crown of the head in both sexes and the left frontal in specifically males, tended to show more variability in the underlying cortical regions that would be measured from fNIRS sensors. For example, based on the results shown in Fig. 4, fNIRS sensors positioned on 10-5 location Fp1 (left superior frontal) in two subjects would, on average, be measuring from two different cortical regions displaced by $3 \mathrm{~cm}$ in registered cortical space. Conversely, regions along the lateral frontal area (e.g., around AF8/7-F8/7) are more conserved across subjects; fNIRS measurements from these regions would be expected to come from more similar cortical regions. This implies that studies focused on group-level analyses from these more variable regions should expect a lower effect size in brain activation and consequently should plan on larger sample sizes to counteract the variability in the cortical regions relative to the fNIRS sensors. Fortunately, we found that this effect was not sex 
Table 4 Nearest 10-5 location for recording Brodmann areas (both sexes).

\begin{tabular}{|c|c|c|c|c|c|c|c|c|}
\hline \multirow[b]{2}{*}{$\mathrm{BA}$} & \multirow[b]{2}{*}{ Region } & \multirow{2}{*}{$\frac{\text { Depth }}{\operatorname{Med}(\min \text { to } \max )}$} & \multicolumn{2}{|c|}{ Position 1} & \multicolumn{2}{|c|}{ Position 2} & \multicolumn{2}{|c|}{ Position 3} \\
\hline & & & Name & Depth & Name & Depth & Name & Depth \\
\hline \multicolumn{9}{|c|}{ Left hemisphere } \\
\hline $\mathrm{BA}-1$ & Prim. somatosensory ctx & 16.85 (6.31 to 26.80$)$ & C3h & 18.13 & C3 & 18.72 & C1 & 21.37 \\
\hline BA-2 & Prim. somatosensory ctx & 19.50 (10.24 to 26.80$)$ & $\mathrm{C} 3$ & 18.99 & $\mathrm{C} 3 \mathrm{~h}$ & 20.78 & $\mathrm{C} 1$ & 22.74 \\
\hline BA-3 & Prim. somatosensory ctx & 20.71 (9.70 to 27.58 ) & C1h & 20.56 & $\mathrm{C} 1$ & 22.47 & C3h & 23.33 \\
\hline BA-4 & Prim. motor ctx & 17.81 (9.90 to 26.61 ) & FCC5h & 18.68 & C1h & 19.14 & FCC3 & 20.32 \\
\hline BA-6 & Motor and suppl. motor ctx & 20.65 (10.38 to 25.98$)$ & FCC1h & 21.65 & FCC1 & 22.58 & $\mathrm{FCCz}$ & 26.47 \\
\hline BA-7 & Somatosensory assoc. ctx & 23.45 (19.07 to 29.61$)$ & $\mathrm{CP} 1$ & 21.79 & CP1h & 23.56 & CCP1h & 24.44 \\
\hline BA-8 & Includes frontal eye fields & 17.94 (7.31 to 24.82$)$ & $\mathrm{F} 1 \mathrm{~h}$ & 18.07 & FFC1h & 18.92 & FFC1 & 19.12 \\
\hline BA-9 & Dorsolateral prefrontal ctx & 20.26 (12.06 to 27.22 ) & $\mathrm{F} 1$ & 19.51 & FC3 & 23.68 & FCC3 & 23.76 \\
\hline $\mathrm{BA}-10$ & Frontopolar area & 19.02 (14.97 to 25.12$)$ & F3 & 17.54 & AFF1h & 24.22 & AFF1 & 24.30 \\
\hline BA-17 & Prim. visual ctx (V1) & 16.66 (11.62 to 26.78$)$ & POO1 & 16.52 & PO1 & 18.31 & P01h & 18.93 \\
\hline BA-18 & Visual assoc. ctx (V2) & 18.50 (15.18 to 24.39$)$ & PPO5h & 17.29 & PPO1h & 19.21 & PO1h & 21.61 \\
\hline BA-19 & Associative visual ctx (V3) & 20.02 (15.59 to 24.85$)$ & P3h & 19.07 & CPP3 & 20.58 & CPP5h & 21.42 \\
\hline BA-21 & Middle temporal gyrus & 21.62 (16.54 to 28.05$)$ & CCP5 & 22.42 & C5 & 24.26 & FCC5 & 25.33 \\
\hline BA-22 & Superior temporal gyrus & 24.51 (20.95 to 28.74$)$ & CCP5h & 23.06 & FCC5 & 25.16 & FCC5h & 31.26 \\
\hline BA-37 & Fusiform gyrus & 17.33 (11.79 to 24.04$)$ & CPP5 & 18.30 & CPP5h & 18.73 & CP5 & 19.94 \\
\hline BA-39 & Angular gyrus & 18.76 (14.61 to 26.24$)$ & CPP3 & 19.12 & $\mathrm{CP} 3$ & 19.78 & CP3h & 21.71 \\
\hline BA-40 & Supramarginal gyrus & 19.80 (13.52 to 25.98$)$ & CCP3 & 19.76 & CCP3h & 21.97 & C3h & 22.80 \\
\hline BA-43 & Subcentral area & 14.70 (4.84 to 24.00$)$ & $\mathrm{C} 5 \mathrm{~h}$ & 15.29 & FCC5h & 16.58 & FCC3 & 22.35 \\
\hline BA-45 & Pars triangularis & 16.35 (12.02 to 24.41$)$ & FC5h & 16.93 & FFC5h & 20.04 & FC3 & 20.80 \\
\hline BA-46 & Dorsolateral prefrontal ctx & 17.49 (10.73 to 24.16$)$ & $\mathrm{FFC3}$ & 18.11 & FFC5h & 18.47 & FC3 & 18.53 \\
\hline \multicolumn{9}{|c|}{ Right hemisphere } \\
\hline BA-1 & Prim. somatosensory ctx & $16.74(10.31$ to 22.10$)$ & $\mathrm{C} 4$ & 16.72 & $\mathrm{C} 4 \mathrm{~h}$ & 18.95 & $\mathrm{C} 2$ & 20.88 \\
\hline BA-2 & Prim. somatosensory ctx & 18.49 (12.28 to 25.25$)$ & $\mathrm{C} 4$ & 16.28 & $\mathrm{C} 4 \mathrm{~h}$ & 20.79 & $\mathrm{C} 2$ & 21.86 \\
\hline BA-3 & Prim. somatosensory ctx & 17.69 (9.79 to 24.37$)$ & $\mathrm{C} 4 \mathrm{~h}$ & 17.40 & $\mathrm{C} 2$ & 21.80 & $\mathrm{C} 2 \mathrm{~h}$ & 22.37 \\
\hline BA-4 & Prim. motor ctx & 18.65 (9.95 to 25.18$)$ & $\mathrm{C} 2 \mathrm{~h}$ & 19.95 & FCC4h & 23.55 & $\mathrm{C} 4 \mathrm{~h}$ & 24.47 \\
\hline BA-6 & Motor and suppl. motor ctx & $19.04(10.36$ to 24.12$)$ & FCC4 & 17.96 & FCC2h & 21.64 & FCC2 & 22.34 \\
\hline BA-7 & Somatosensory assoc. ctx & 22.87 (16.07 to 30.78$)$ & $\mathrm{CP} 2 \mathrm{~h}$ & 22.41 & $\mathrm{CP} 2$ & 22.53 & CCP2h & 24.76 \\
\hline BA-8 & Includes frontal eye fields & 18.83 (11.15 to 24.62$)$ & FC4h & 19.64 & FFC2h & 20.76 & FFC2 & 21.20 \\
\hline BA-9 & Dorsolateral prefrontal ctx & 19.45 (13.05 to 25.82 ) & $\mathrm{FC} 4 \mathrm{~h}$ & 22.08 & FC4 & 22.28 & $\mathrm{FCC} 4$ & 22.83 \\
\hline $\mathrm{BA}-10$ & Frontopolar area & 18.08 (15.34 to 23.34$)$ & $\mathrm{F} 4$ & 17.39 & AFF2 & 20.89 & AFF2h & 22.54 \\
\hline BA-17 & Prim. visual ctx (V1) & 18.20 (12.11 to 27.42$)$ & $\mathrm{PO} 4 \mathrm{~h}$ & 17.04 & PO2 & 17.93 & PO2h & 18.18 \\
\hline BA-18 & Visual assoc. ctx (V2) & $18.47(14.47$ to 25.04$)$ & PPO4 & 16.28 & PPO2h & 19.96 & $\mathrm{PO} 2 \mathrm{~h}$ & 20.60 \\
\hline
\end{tabular}


Table 4 (Continued).

\begin{tabular}{|c|c|c|c|c|c|c|c|c|}
\hline \multirow[b]{2}{*}{ BA } & \multirow[b]{2}{*}{ Region } & \multirow{2}{*}{$\frac{\text { Depth }}{\operatorname{Med}(\min \text { to } \max )}$} & \multicolumn{2}{|c|}{ Position 1} & \multicolumn{2}{|c|}{ Position 2} & \multicolumn{2}{|c|}{ Position 3} \\
\hline & & & Name & Depth & Name & Depth & Name & Depth \\
\hline BA-19 & Associative visual ctx (V3) & 19.05 (15.81 to 24.77$)$ & $\mathrm{P} 4 \mathrm{~h}$ & 19.07 & $\mathrm{P} 2$ & 20.33 & $\mathrm{P} 2 \mathrm{~h}$ & 22.70 \\
\hline BA-21 & Middle temporal gyrus & $19.23(13.02$ to 25.89$)$ & CCP6 & 19.31 & C6 & 20.73 & FCC6 & 23.57 \\
\hline BA-22 & Superior temporal gyrus & 23.32 (16.57 to 29.15$)$ & CP6h & 21.26 & CCP6h & 22.62 & FCC6h & 26.80 \\
\hline BA-37 & Fusiform gyrus & 17.75 (11.57 to 23.92$)$ & CP6 & 17.18 & CPP6 & 19.31 & CPP6h & 20.76 \\
\hline BA-39 & Angular gyrus & 18.70 (12.57 to 25.00$)$ & CPP4 & 19.64 & CP4 & 19.99 & $\mathrm{CP} 4 \mathrm{~h}$ & 20.00 \\
\hline BA-40 & Supramarginal gyrus & 20.83 (14.98 to 26.12 ) & $\mathrm{CCP} 4$ & 18.95 & CCP4h & 22.86 & C4h & 23.32 \\
\hline BA-43 & Subcentral area & 13.79 (5.01 to 26.59$)$ & C6h & 16.05 & $\mathrm{C} 4$ & 17.64 & $\mathrm{FCC} 4$ & 20.58 \\
\hline BA-45 & Pars triangularis & 14.48 (6.86 to 22.95$)$ & FC6h & 15.80 & FC4 & 17.26 & FFC6h & 17.68 \\
\hline BA-46 & Dorsolateral prefrontal ctx & 16.83 (10.10 to 23.75$)$ & FFC6h & 17.63 & FFC4 & 18.80 & $\mathrm{FC} 4$ & 19.17 \\
\hline
\end{tabular}

Note: This table provides the depth (median and range) of selected Brodmann areas based on the aal template in MNI space. For each region, the nearest three 10-5 coordinate positions and the depth of the region to this position are provided as guidance for the placement of fNIRS sensors. The individual results for the two genders are presented in the Appendix to this work.

specific and both males and females had similar areas of variability (Fig. 4). This means that sex is not expected to affect precision of fNIRS measurements, although we found evidence of a clear bias in the magnitude (accuracy) of the measurements due to cortical depth.

\subsection{Effects of Variations on Optical Properties}

Based on the segmented structural MRI data, we also ran Monte Carlo simulations to look at how PPF and DPF varied spatially and across age and sex. The regions that showed the greatest variations in cortical depth between sexes were also the most variable in the partial pathlength (PPF). Since optical PPF is defined as the multiplication factor to estimate the pathlength through specifically the brain [e.g., $\quad L_{\text {brain }}=\mathrm{PPF} \times L_{\text {source-detector }}$; Eq. (2)] within the MBLL, these spatial variations have a direct impact on the quantitative report of the magnitude of estimated brain signals. In particular, we found about a $13 \%$ to $26 \%$ spatial difference going from the frontal or lateral regions of the head to superior regions (Fig. 7). This means that for the same change in hemoglobin within these brain regions, the measured optical signals in the lateral regions would predict a lower magnitude change compared to those from near the top of the head. We also noted significant sex differences in the optical PPF, particularly in the frontal cortex (Fig. 7). Specifically, the females had $\sim 13 \%$ to $18 \%$ smaller PPF values compared to the males. This seems to be the effect of the cortical depth of about 2 to $3 \mathrm{~mm}$ in the females in this region and we believe reflects a slightly larger sinus cavity at the front of the head. Unfortunately, this PPF difference implies that for the same underlying magnitude of hemoglobin changes in the brain, the optical measurements in the females are expected to be smaller. This introduces a systematic bias in group-level statistical analysis, which looks at sex as a covariate. Since the dominant source of noise in fNIRS measurements is probably physiological signals in the skin layers, which would be expected to be similar in both sexes, the lower PPF likely directly relates to a lower contrast-to-noise ratio and lower statistical effect sizes in this group. Even if a sex-adjusted DPF is applied in analysis, this difference in the expected statistical effect would have an effect on group-size and power estimates for analysis. Optical DPF did not have nearly as much spatial variation and showed only very little variation across the same regions. DPF is the multiplication factor between the source-detector distance and the total pathlength through the tissue. Although DPF can be measured directly by time-domain or frequency-domain fNIRS technologies, PPF cannot and must be modeled.

Although DPF and PPF varied over space, within a given region, they do not vary much across the five optical wavelengths examined in this work. This means that the potential for crosstalk in the separation of $\mathrm{HbO}_{2}$ and $\mathrm{Hb}$ does not seem to vary across space, although we did not explicitly model this factor. The work by Strangman et al. ${ }^{24}$ examined the effect of cross talk in the separation of $\mathrm{HbO}_{2}$ and $\mathrm{Hb}$ in the choice of wavelengths and how this cross-talk is affected by differences in the DPF/PPF values at different wavelengths. Our current work seems consistent with their findings in that we did not see much evidence that the level of cross-talk would be expected to vary much across space or by subject age/sex.

\subsection{Guidance for Designing Future Studies}

Tables 3 and 4 as well as Tables 5-15 in the appendix can be used to design future fNIRS studies. Table 3 provides estimates of the relative sensitivities of various brain regions to fNIRS measurements taken from the normalized count of photons able to reach these brain regions. This depended on both the spatial extent and depth of these regions. As a general guidance, based on our own experience, we believe that regions down to about $-40 \mathrm{~dB}$ are fNIRS-accessible based on current instrument sensitivities. For example, the TechEN Inc. (Milford MA) system has an instrument noise floor of about $68 \mathrm{~dB} .{ }^{30}$ Table 3 provides these values for the FreeSurfer anatomical parcellations of the cortex for a source-detector spacing of $30 \mathrm{~mm}$. Tables 5-15 for Brodmann areas and by sex are also presented in the Appendix. Likewise, Table 4 provides a list of the nearest 10-5 
head coordinate point to specific accessible Brodmann areas, which could be used to guide head cap designs for future studies.

\subsection{Limitations of this Study}

In this study, we used Monte Carlo modeling of the fNIRS forward solution to attempt to quantify the effects of head anatomy in this population of 5- to 11-year-old children. One limitation of this work is that our results depend on the parameters and optical properties that we used in these simulations. In particular, we used bulk optical properties for the skin, skull, CSF, and brain [gray/white] layers, but did not consider any subject or spatial variability in these values. Although both gray and white matters were segmented, the optical properties used for these two layers (Table 2) were the same. In addition, there is considerable variability in these values in the literature. ${ }^{25}$ Several previous papers have attempted to experimentally quantify these values using time-resolved or frequency-domain fNIRS methods. Duncan et al. ${ }^{31}$ used frequency-domain fNIRS on 283 subjects (ages 0 to 50 year old) in the frontal-temporal regions and examined the relationship of DPF with subject age. In that work, an equation was derived to predict DPF as a function of subject age. Additional work by Cooper $^{32}$ examined DPF in neonates and several papers have also examined this in adults. ${ }^{13,14,33-36}$ The work by Scholkmann and Wolf ${ }^{37}$ provides a review and summary of these methods and offers a generalized model for the DPF in the frontal cortex. In comparison to this previous literature, our results are generally consistent with DPF being between about 4 to 6.5 . Of note however, the work by
Bonnery et al. ${ }^{14}$ did suggest some discrepancies between timeresolved experimental fNIRS measurements of DPF and Monte Carlo simulations. Therefore, we caution anyone trying to use the values provided in this work to provide quantitative accuracy. This work is intended as guidance for the design and interpretation of fNIRS studies in school-aged children. For example, we used the same optical absorption and scattering for all simulations, but it is possible that these values might change over age or the values used may offer some bias in the results. This study is not intended to substitute for direct measurements of DPF and optical properties that could be obtained by timedomain or frequency-domain fNIRS techniques. Further experimental work is still needed to verify these results.

A second limitation of this work is that our analysis of DPF and PPF in this study was based on the collection of data from simulated measurements of emitter-detectors spacing from 10 to $40 \mathrm{~mm}$. The MBLL [Eq. (2)] assumes that the pathlength correction is linear with this spacing, however, this is probably not entirely true in regions with thick CSF layers such as the frontal pole. In particular, for short spacings or areas where the brain is deeper, the PPF term would be particularly sensitive to nonlinearities.

\section{Appendix:}

Provided below are the tables describing the results of the Monte-Carlo simulations. Tables 5-14 provide information by either Brodmann area or anatomical region.

Table 5 Sensitivity, DPF, and PPF of select Brodmann areas (both sexes).

\begin{tabular}{|c|c|c|c|c|c|c|c|c|c|c|c|c|}
\hline \multirow[b]{2}{*}{ BA } & \multirow[b]{2}{*}{ Region } & \multirow[b]{2}{*}{ Sens. } & \multicolumn{5}{|c|}{$\mathrm{DPF}(\mathrm{nm})$} & \multicolumn{5}{|c|}{ PPF (nm) } \\
\hline & & & 690 & 780 & 808 & 830 & 850 & 690 & 780 & 808 & 830 & 850 \\
\hline BA-1 & L prim. somatosensory ctx & -42.9 & 6.11 & 5.95 & 5.90 & 5.89 & 5.88 & 2.67 & 2.75 & 2.79 & 2.77 & 2.84 \\
\hline BA-1 & R prim. somatosensory ctx & -36.4 & 6.13 & 5.91 & 5.88 & 5.78 & 5.78 & 1.78 & 1.85 & 1.86 & 1.86 & 1.91 \\
\hline BA-2 & L prim. somatosensory ctx & -37.1 & 6.13 & 5.94 & 5.90 & 5.89 & 5.86 & 2.62 & 2.66 & 2.71 & 2.66 & 2.73 \\
\hline BA-2 & R prim. somatosensory ctx & -35.5 & 6.11 & 5.88 & 5.79 & 5.76 & 5.74 & 1.87 & 1.90 & 1.96 & 1.96 & 2.08 \\
\hline BA-3 & L prim. somatosensory ctx & -40.0 & 6.12 & 5.95 & 5.92 & 5.87 & 5.85 & 2.62 & 2.69 & 2.70 & 2.72 & 2.75 \\
\hline BA-3 & R prim. somatosensory ctx & -35.0 & 6.13 & 5.87 & 5.83 & 5.79 & 5.77 & 1.96 & 1.99 & 2.04 & 2.01 & 2.07 \\
\hline BA-4 & L prim. motor ctx & -40.6 & 6.14 & 6.00 & 5.95 & 5.88 & 5.92 & 2.60 & 2.66 & 2.72 & 2.71 & 2.74 \\
\hline BA-4 & R prim. motor ctx & -41.2 & 6.08 & 5.83 & 5.78 & 5.76 & 5.73 & 1.81 & 1.85 & 1.87 & 1.89 & 1.94 \\
\hline BA-6 & L motor and suppl. motor ctx & -34.2 & 6.18 & 5.99 & 5.97 & 5.93 & 5.91 & 2.62 & 2.55 & 2.59 & 2.59 & 2.67 \\
\hline BA-6 & $\mathrm{R}$ motor and suppl. motor ctx & -27.9 & 6.16 & 5.93 & 5.92 & 5.85 & 5.85 & 1.90 & 1.94 & 1.94 & 1.96 & 2.02 \\
\hline BA-7 & L somatosensory assoc. ctx & -36.5 & 6.03 & 5.80 & 5.75 & 5.72 & 5.68 & 2.33 & 2.29 & 2.35 & 2.32 & 2.37 \\
\hline BA-7 & $\mathrm{R}$ somatosensory assoc. ctx & -37.2 & 6.03 & 5.78 & 5.73 & 5.71 & 5.69 & 2.05 & 2.07 & 2.07 & 2.13 & 2.14 \\
\hline BA-8 & $L$ includes frontal eye fields & -33.4 & 6.20 & 6.04 & 5.95 & 5.94 & 5.93 & 2.24 & 2.32 & 2.33 & 2.32 & 2.39 \\
\hline BA-8 & $R$ includes frontal eye fields & -35.8 & 6.29 & 6.07 & 6.05 & 6.01 & 5.96 & 1.92 & 2.01 & 2.02 & 2.01 & 2.05 \\
\hline
\end{tabular}


Table 5 (Continued).

\begin{tabular}{|c|c|c|c|c|c|c|c|c|c|c|c|c|}
\hline \multirow[b]{2}{*}{ BA } & \multirow[b]{2}{*}{ Region } & \multirow[b]{2}{*}{ Sens. } & \multicolumn{5}{|c|}{$\operatorname{DPF}(\mathrm{nm})$} & \multicolumn{5}{|c|}{ PPF (nm) } \\
\hline & & & 690 & 780 & 808 & 830 & 850 & 690 & 780 & 808 & 830 & 850 \\
\hline BA-9 & $\mathrm{L}$ dorsolateral prefrontal ctx & -34.5 & 6.15 & 5.95 & 5.93 & 5.95 & 5.91 & 2.43 & 2.48 & 2.52 & 2.49 & 2.51 \\
\hline BA-9 & $\mathrm{R}$ dorsolateral prefrontal ctx & -39.6 & 6.31 & 6.04 & 5.98 & 5.93 & 5.91 & 1.72 & 1.74 & 1.77 & 1.78 & 1.78 \\
\hline BA-10 & $\mathrm{L}$ frontopolar area & -30.7 & 6.04 & 5.86 & 5.82 & 5.76 & 5.74 & 1.98 & 1.91 & 1.92 & 1.95 & 2.04 \\
\hline BA-10 & $\mathrm{R}$ frontopolar area & -30.8 & 6.22 & 5.99 & 5.91 & 5.85 & 5.81 & 1.55 & 1.56 & 1.57 & 1.61 & 1.59 \\
\hline BA-17 & L prim. visual ctx (V1) & -39.6 & 5.99 & 5.73 & 5.70 & 5.64 & 5.61 & 1.99 & 1.93 & 1.97 & 1.97 & 2.05 \\
\hline BA-17 & R prim. visual ctx (V1) & -41.7 & 5.97 & 5.71 & 5.63 & 5.58 & 5.56 & 1.62 & 1.66 & 1.64 & 1.66 & 1.70 \\
\hline BA-18 & L visual assoc. ctx (V2) & -28.7 & 6.03 & 5.75 & 5.70 & 5.64 & 5.59 & 2.15 & 2.14 & 2.18 & 2.19 & 2.22 \\
\hline BA-18 & $\mathrm{R}$ visual assoc. ctx (V2) & -27.9 & 5.94 & 5.66 & 5.61 & 5.54 & 5.51 & 1.56 & 1.57 & 1.59 & 1.64 & 1.73 \\
\hline BA-19 & $\mathrm{L}$ associative visual ctx (V3) & -30.3 & 5.98 & 5.77 & 5.72 & 5.68 & 5.65 & 2.43 & 2.39 & 2.41 & 2.42 & 2.53 \\
\hline BA-19 & $\mathrm{R}$ associative visual ctx (V3) & -31.8 & 5.93 & 5.68 & 5.62 & 5.59 & 5.56 & 2.00 & 1.96 & 1.98 & 2.00 & 2.10 \\
\hline BA-21 & $\mathrm{L}$ middle temporal gyrus & -37.2 & 6.08 & 5.90 & 5.84 & 5.81 & 5.80 & 2.58 & 2.64 & 2.63 & 2.59 & 2.58 \\
\hline BA-21 & $\mathrm{R}$ middle temporal gyrus & -34.0 & 6.01 & 5.80 & 5.77 & 5.73 & 5.69 & 1.68 & 1.61 & 1.64 & 1.67 & 1.76 \\
\hline BA-22 & L superior temporal gyrus & -38.9 & 6.09 & 5.94 & 5.91 & 5.89 & 5.86 & 2.69 & 2.72 & 2.76 & 2.70 & 2.74 \\
\hline BA-22 & R superior temporal gyrus & -36.0 & 6.07 & 5.86 & 5.82 & 5.79 & 5.74 & 1.71 & 1.73 & 1.76 & 1.78 & 1.78 \\
\hline BA-37 & L fusiform gyrus & -37.9 & 5.97 & 5.76 & 5.70 & 5.67 & 5.63 & 2.38 & 2.35 & 2.40 & 2.45 & 2.52 \\
\hline BA-37 & $R$ fusiform gyrus & -34.3 & 5.96 & 5.70 & 5.64 & 5.61 & 5.58 & 1.74 & 1.73 & 1.77 & 1.83 & 1.83 \\
\hline BA-39 & $\mathrm{L}$ angular gyrus & -35.7 & 5.97 & 5.77 & 5.73 & 5.70 & 5.68 & 2.25 & 2.29 & 2.31 & 2.36 & 2.44 \\
\hline BA-39 & $\mathrm{R}$ angular gyrus & -36.5 & 5.96 & 5.72 & 5.65 & 5.65 & 5.61 & 1.86 & 1.92 & 1.92 & 1.98 & 2.02 \\
\hline BA-40 & L supramarginal gyrus & -32.9 & 6.05 & 5.83 & 5.81 & 5.77 & 5.76 & 2.51 & 2.54 & 2.57 & 2.58 & 2.63 \\
\hline BA-40 & R supramarginal gyrus & -31.0 & 6.03 & 5.83 & 5.79 & 5.75 & 5.72 & 1.72 & 1.75 & 1.77 & 1.82 & 1.84 \\
\hline BA-43 & $\mathrm{L}$ subcentral area & -37.7 & 6.15 & 6.02 & 5.99 & 5.97 & 5.99 & 2.71 & 2.75 & 2.76 & 2.76 & 2.83 \\
\hline BA-43 & $\mathrm{R}$ subcentral area & -40.0 & 6.08 & 5.86 & 5.84 & 5.79 & 5.76 & 1.75 & 1.78 & 1.82 & 1.84 & 1.83 \\
\hline BA-45 & $\mathrm{L}$ pars triangularis & -39.6 & 6.15 & 5.98 & 5.95 & 5.90 & 5.91 & 2.61 & 2.63 & 2.65 & 2.62 & 2.68 \\
\hline BA-45 & $\mathrm{R}$ pars triangularis & -39.4 & 6.30 & 6.07 & 6.01 & 5.96 & 5.93 & 1.77 & 1.83 & 1.81 & 1.86 & 1.86 \\
\hline BA-46 & $\mathrm{L}$ dorsolateral prefrontal ctx & -36.4 & 6.17 & 5.97 & 5.94 & 5.89 & 5.89 & 2.48 & 2.52 & 2.52 & 2.48 & 2.50 \\
\hline BA-46 & $\mathrm{R}$ dorsolateral prefrontal ctx & -36.6 & 6.29 & 6.02 & 5.96 & 5.94 & 5.88 & 1.63 & 1.64 & 1.67 & 1.71 & 1.77 \\
\hline
\end{tabular}

Note: This table shows the differential (DPF) and PPF for several fNIRS-accessible Brodmann areas in the head. Data have been combined for both male and female subjects. The sensitivity of the region to fNIRS is provided in decibels $(\mathrm{dB} ; 2710 \times \log 10)$ and indicates the fraction of photons normalized to the incidence power reaching this region from the scalp at a source-detector distance of $30 \mathrm{~mm}$. 
Table 6 Nearest 10-5 location for recording select anatomical regions (both sexes).

\begin{tabular}{|c|c|c|c|c|c|c|c|}
\hline \multirow[b]{2}{*}{ Region } & \multirow{2}{*}{$\frac{\text { Depth }}{\operatorname{Med}(\min \text { to } \max )}$} & \multicolumn{2}{|c|}{ Position 1} & \multicolumn{2}{|c|}{ Position 2} & \multicolumn{2}{|c|}{ Position 3} \\
\hline & & Name & Depth & Name & Depth & Name & Depth \\
\hline L caudal middle frontal & 20.33 (11.99 to 26.63 ) & FC3h & 19.50 & FCC3h & 21.01 & FC3 & 25.10 \\
\hline $\mathrm{R}$ caudal middle frontal & $20.46(11.36$ to 25.70$)$ & FC2 & 20.20 & FCC2 & 20.43 & FC4h & 22.09 \\
\hline$L$ frontal pole & $14.70(10.80$ to 21.25$)$ & AFp1 & 14.68 & AFp3h & 14.73 & Fp1h & 16.59 \\
\hline $\mathrm{R}$ frontal pole & 15.34 (12.08 to 22.68$)$ & AFp4h & 15.30 & AFp2h & 15.80 & Fp2h & 16.95 \\
\hline $\mathrm{L}$ inferior parietal & 18.20 (13.25 to 22.86$)$ & СРP3h & 17.98 & CPP3 & 20.18 & CPP5h & 23.19 \\
\hline $\mathrm{R}$ inferior parietal & 17.93 (13.18 to 23.42$)$ & $\mathrm{CP} 4 \mathrm{~h}$ & 19.01 & CPP4 & 19.76 & $\mathrm{CP} 4$ & 20.03 \\
\hline$L$ inferior temporal & 20.05 (15.60 to 28.07$)$ & CP5 & 18.81 & CPP5 & 19.88 & TP7h & 21.93 \\
\hline $\mathrm{R}$ inferior temporal & 19.70 (13.89 to 27.26$)$ & CPP6 & 18.68 & CP6 & 18.78 & T8h & 26.15 \\
\hline L lateral occipital & 14.15 (11.12 to 21.28$)$ & PPO5h & 15.33 & PPO3 & 16.59 & P5h & 18.59 \\
\hline $\mathrm{R}$ lateral occipital & 14.09 (11.54 to 21.02$)$ & PPO4h & 16.03 & PPO4 & 16.07 & P6h & 17.84 \\
\hline L middle temporal & $16.19(12.21$ to 21.67$)$ & CP5 & 15.68 & C5 & 20.58 & CCP5 & 20.67 \\
\hline $\mathrm{R}$ middle temporal & 16.27 (9.52 to 21.80$)$ & CCP6 & 18.15 & C6 & 19.15 & FTT8h & 22.78 \\
\hline L pars orbitalis & 18.15 (13.52 to 29.05$)$ & F5 & 18.28 & FFC5 & 19.73 & FC5 & 20.81 \\
\hline $\mathrm{R}$ pars orbitalis & 17.86 (13.32 to 28.56$)$ & F6h & 17.99 & F6 & 18.67 & FFC6 & 19.17 \\
\hline $\mathrm{L}$ pars triangularis & 17.64 (13.52 to 24.87$)$ & FFC5 & 18.56 & FC5h & 18.59 & FFC5h & 21.27 \\
\hline $\mathrm{R}$ pars triangularis & $17.11(10.76$ to 26.09$)$ & FC6h & 17.82 & FFC4 & 19.32 & FFC6h & 20.21 \\
\hline L postcentral & $18.22(10.85$ to 26.28$)$ & $\mathrm{C} 3$ & 18.75 & C3h & 19.41 & $\mathrm{C} 5 \mathrm{~h}$ & 20.42 \\
\hline R postcentral & $18.11(11.33$ to 24.70$)$ & $\mathrm{C} 4$ & 17.97 & $\mathrm{C} 4 \mathrm{~h}$ & 19.76 & $\mathrm{C} 2$ & 21.15 \\
\hline L precentral & 20.46 (11.63 to 26.01$)$ & FCC5h & 20.29 & $\mathrm{FCC} 3$ & 20.41 & $\mathrm{C} 1$ & 22.97 \\
\hline $\mathrm{R}$ precentral & 20.27 (12.58 to 26.21$)$ & $\mathrm{FCC} 4$ & 19.37 & $\mathrm{FCC} 4 \mathrm{~h}$ & 20.50 & $\mathrm{C} 2$ & 23.69 \\
\hline $\mathrm{L}$ rostral middle frontal & $16.29(11.35$ to 21.98$)$ & FFC3 & 18.19 & FFC3h & 18.84 & $\mathrm{~F} 3 \mathrm{~h}$ & 19.07 \\
\hline $\mathrm{R}$ rostral middle frontal & $17.21(11.97$ to 22.37$)$ & FFC4 & 19.11 & $\mathrm{FC} 4$ & 19.37 & $\mathrm{FFC} 4 \mathrm{~h}$ & 19.56 \\
\hline $\mathrm{L}$ superior frontal & $20.57(10.86$ to 27.77$)$ & FFC1h & 19.70 & $\mathrm{FC} 1 \mathrm{~h}$ & 21.96 & FCC1h & 23.38 \\
\hline $\mathrm{R}$ superior frontal & 21.69 (12.48 to 27.22$)$ & FFC2h & 21.41 & $\mathrm{FC} 2 \mathrm{~h}$ & 22.46 & $\mathrm{FCC} 2 \mathrm{~h}$ & 22.80 \\
\hline L superior parietal & 20.97 (16.07 to 28.94$)$ & $\mathrm{CP} 1$ & 22.01 & CCP1 & 22.59 & CPP1 & 22.68 \\
\hline $\mathrm{R}$ superior parietal & 20.93 (16.19 to 27.98$)$ & CP2h & 20.94 & $\mathrm{CP} 2$ & 23.51 & $\mathrm{CCP} 2$ & 24.15 \\
\hline L superior temporal & 22.43 (18.26 to 27.88$)$ & FCC5 & 23.85 & C5 & 23.96 & CCP5h & 24.42 \\
\hline $\mathrm{R}$ superior temporal & $22.26(13.63$ to 28.74$)$ & CCP6h & 22.24 & FCC6 & 23.81 & C6 & 25.09 \\
\hline
\end{tabular}

Note: This table provides the depth (median and range) of selected FreeSurfer anatomical regions. Data have been combined for both male and female subjects. For each region, the nearest three 10-5 coordinate positions and the depth of the region to this position is provided as guidance for the placement of fNIRS sensors. 
Table 7 Sensitivity, DPF, PPF of Brodmann areas (females).

\begin{tabular}{|c|c|c|c|c|c|c|c|c|c|c|c|c|}
\hline \multirow[b]{2}{*}{ BA } & \multirow[b]{2}{*}{ Region } & \multirow[b]{2}{*}{ Sens. } & \multicolumn{5}{|c|}{$\mathrm{DPF}(\mathrm{nm})$} & \multicolumn{5}{|c|}{ PPF (nm) } \\
\hline & & & 690 & 780 & 808 & 830 & 850 & 690 & 780 & 808 & 830 & 850 \\
\hline BA-1 & L prim. somatosensory ctx & -44.1 & 6.06 & 5.91 & 5.88 & 5.87 & 5.82 & 2.49 & 2.52 & 2.54 & 2.55 & 2.66 \\
\hline BA-1 & R prim. somatosensory ctx & -36.2 & 6.08 & 5.90 & 5.79 & 5.78 & 5.78 & 1.63 & 1.73 & 1.71 & 1.76 & 1.81 \\
\hline BA-2 & L prim. somatosensory ctx & -36.4 & 6.10 & 5.93 & 5.88 & 5.87 & 5.85 & 2.42 & 2.49 & 2.48 & 2.52 & 2.60 \\
\hline BA-2 & R prim. somatosensory ctx & -34.7 & 6.05 & 5.84 & 5.80 & 5.77 & 5.81 & 1.82 & 1.88 & 1.95 & 1.95 & 2.03 \\
\hline BA-3 & L prim. somatosensory ctx & -43.1 & 6.11 & 5.91 & 5.89 & 5.82 & 5.82 & 2.40 & 2.45 & 2.41 & 2.47 & 2.59 \\
\hline BA-3 & R prim. somatosensory ctx & -36.3 & 6.13 & 5.85 & 5.84 & 5.79 & 5.80 & 1.90 & 1.92 & 1.99 & 1.97 & 2.03 \\
\hline BA-4 & L prim. motor ctx & -41.4 & 6.10 & 5.90 & 5.87 & 5.84 & 5.81 & 2.40 & 2.49 & 2.44 & 2.52 & 2.61 \\
\hline BA-4 & R prim. motor ctx & -41.3 & 5.98 & 5.79 & 5.76 & 5.75 & 5.75 & 1.72 & 1.77 & 1.81 & 1.85 & 1.86 \\
\hline BA-5 & L somatosensory assoc. ctx & -44.7 & 6.08 & 5.84 & 5.78 & 5.76 & 5.73 & 2.00 & 2.03 & 2.12 & 2.13 & 2.20 \\
\hline BA-5 & $\mathrm{R}$ somatosensory assoc. ctx & -44.9 & 6.06 & 5.80 & 5.76 & 5.73 & 5.74 & 1.86 & 1.94 & 1.90 & 2.02 & 2.11 \\
\hline BA-6 & L motor and suppl. motor ctx & -35.3 & 6.18 & 5.97 & 5.96 & 5.93 & 5.88 & 2.21 & 2.43 & 2.44 & 2.46 & 2.52 \\
\hline BA-6 & $\mathrm{R}$ motor and suppl. motor ctx & -27.2 & 6.21 & 5.97 & 5.91 & 5.87 & 5.88 & 1.75 & 1.84 & 1.86 & 1.88 & 1.94 \\
\hline BA-7 & L somatosensory assoc. ctx & -37.5 & 5.96 & 5.73 & 5.70 & 5.64 & 5.60 & 2.13 & 2.13 & 2.15 & 2.19 & 2.23 \\
\hline BA-7 & R somatosensory assoc. ctx & -37.3 & 6.02 & 5.73 & 5.67 & 5.63 & 5.60 & 1.86 & 2.00 & 1.96 & 2.07 & 2.12 \\
\hline BA-8 & $L$ includes frontal eye fields & -34.0 & 6.18 & 5.98 & 5.94 & 5.90 & 5.91 & 1.93 & 2.03 & 2.04 & 2.09 & 2.16 \\
\hline BA-8 & $R$ includes frontal eye fields & -35.7 & 6.35 & 6.09 & 6.06 & 6.02 & 5.96 & 1.64 & 1.68 & 1.70 & 1.74 & 1.73 \\
\hline BA-9 & $\mathrm{L}$ dorsolateral prefrontal ctx & -34.1 & 6.08 & 5.88 & 5.88 & 5.82 & 5.86 & 2.40 & 2.36 & 2.43 & 2.38 & 2.47 \\
\hline BA-9 & $\mathrm{R}$ dorsolateral prefrontal ctx & -39.5 & 6.35 & 6.03 & 5.98 & 5.93 & 5.91 & 1.46 & 1.57 & 1.57 & 1.60 & 1.65 \\
\hline BA-10 & $\mathrm{L}$ frontopolar area & -31.4 & 6.00 & 5.85 & 5.74 & 5.73 & 5.70 & 1.65 & 1.70 & 1.71 & 1.75 & 1.78 \\
\hline $\mathrm{BA}-10$ & $\mathrm{R}$ frontopolar area & -31.1 & 6.21 & 6.00 & 5.92 & 5.85 & 5.82 & 1.34 & 1.41 & 1.41 & 1.40 & 1.39 \\
\hline $\mathrm{BA}-11$ & $\mathrm{~L}$ orbitofrontal area & -58.3 & 6.02 & 5.79 & 5.75 & 5.70 & 5.65 & 1.64 & 1.63 & 1.66 & 1.69 & 1.70 \\
\hline BA-11 & $\mathrm{R}$ orbitofrontal area & -46.0 & 6.26 & 5.93 & 5.87 & 5.87 & 5.81 & 1.19 & 1.18 & 1.18 & 1.21 & 1.24 \\
\hline $\mathrm{BA}-17$ & L prim. visual ctx (V1) & -38.0 & 6.01 & 5.71 & 5.63 & 5.59 & 5.51 & 1.50 & 1.57 & 1.58 & 1.62 & 1.70 \\
\hline BA-17 & R prim. visual ctx (V1) & -41.4 & 6.00 & 5.67 & 5.60 & 5.52 & 5.49 & 1.22 & 1.25 & 1.27 & 1.33 & 1.37 \\
\hline $\mathrm{BA}-18$ & L visual assoc. ctx (V2) & -28.0 & 6.03 & 5.70 & 5.65 & 5.60 & 5.55 & 1.61 & 1.66 & 1.67 & 1.73 & 1.90 \\
\hline BA-18 & $\mathrm{R}$ visual assoc. ctx (V2) & -28.6 & 5.96 & 5.68 & 5.62 & 5.52 & 5.48 & 1.18 & 1.23 & 1.25 & 1.28 & 1.36 \\
\hline BA-19 & $\mathrm{L}$ associative visual ctx (V3) & -32.6 & 6.02 & 5.76 & 5.68 & 5.63 & 5.60 & 1.89 & 1.94 & 2.00 & 2.04 & 2.15 \\
\hline BA-19 & $\mathrm{R}$ associative visual ctx (V3) & -31.4 & 5.94 & 5.65 & 5.59 & 5.58 & 5.54 & 1.68 & 1.71 & 1.72 & 1.75 & 1.79 \\
\hline BA-20 & $\mathrm{L}$ inferior temporal gyrus & -49.6 & 6.02 & 5.90 & 5.86 & 5.82 & 5.84 & 2.39 & 2.51 & 2.53 & 2.53 & 2.56 \\
\hline BA-20 & $\mathrm{R}$ inferior temporal gyrus & -43.0 & 6.00 & 5.75 & 5.72 & 5.66 & 5.64 & 1.80 & 1.76 & 1.77 & 1.71 & 1.81 \\
\hline BA-21 & $\mathrm{L}$ middle temporal gyrus & -36.2 & 6.06 & 5.89 & 5.84 & 5.78 & 5.78 & 2.54 & 2.61 & 2.58 & 2.55 & 2.56 \\
\hline BA-21 & $\mathrm{R}$ middle temporal gyrus & -31.0 & 6.02 & 5.82 & 5.82 & 5.77 & 5.72 & 1.82 & 1.82 & 1.80 & 1.67 & 1.86 \\
\hline BA-22 & L superior temporal gyrus & -39.1 & 6.09 & 5.90 & 5.85 & 5.85 & 5.82 & 2.32 & 2.39 & 2.40 & 2.39 & 2.47 \\
\hline
\end{tabular}


Table 7 (Continued).

\begin{tabular}{|c|c|c|c|c|c|c|c|c|c|c|c|c|}
\hline \multirow[b]{2}{*}{ BA } & \multirow[b]{2}{*}{ Region } & \multirow[b]{2}{*}{ Sens. } & \multicolumn{5}{|c|}{$\mathrm{DPF}(\mathrm{nm})$} & \multicolumn{5}{|c|}{$\mathrm{PPF}(\mathrm{nm})$} \\
\hline & & & 690 & 780 & 808 & 830 & 850 & 690 & 780 & 808 & 830 & 850 \\
\hline BA-22 & R superior temporal gyrus & -36.7 & 6.12 & 5.88 & 5.83 & 5.78 & 5.74 & 1.73 & 1.74 & 1.77 & 1.77 & 1.85 \\
\hline BA-23 & $\mathrm{L}$ ventral post. cingulate ctx & -85.5 & 6.06 & 5.90 & 5.83 & 5.83 & 5.78 & 2.10 & 2.20 & 2.27 & 2.36 & 2.36 \\
\hline BA-23 & $\mathrm{R}$ ventral post. cingulate $\mathrm{ctx}$ & -80.0 & 6.10 & 5.90 & 5.84 & 5.80 & 5.82 & 1.66 & 1.79 & 1.88 & 2.03 & 2.21 \\
\hline BA-24 & $\mathrm{L}$ ventral ant. cingulate $\mathrm{ctx}$ & -74.1 & 6.21 & 6.01 & 5.93 & 5.92 & 5.87 & 2.44 & 2.52 & 2.61 & 2.58 & 2.65 \\
\hline BA-24 & $\mathrm{R}$ ventral ant. cingulate ctx & -68.6 & 6.15 & 5.95 & 5.86 & 5.84 & 5.80 & 1.82 & 1.88 & 1.88 & 1.91 & 2.00 \\
\hline BA-25 & $\mathrm{L}$ subgenual ctx & -78.8 & 6.07 & 5.89 & 5.80 & 5.82 & 5.79 & 1.62 & 1.73 & 1.71 & 1.77 & 1.92 \\
\hline BA-25 & $\mathrm{R}$ subgenual ctx & -67.3 & 6.20 & 6.00 & 5.91 & 5.90 & 5.83 & 1.45 & 1.41 & 1.41 & 1.49 & 1.56 \\
\hline BA-28 & $L$ post. entorhinal ctx & -88.0 & 6.17 & 5.98 & 5.89 & 5.86 & 5.86 & 2.35 & 2.43 & 2.43 & 2.40 & 2.53 \\
\hline BA-28 & R post. entorhinal ctx & -84.3 & 6.08 & 5.83 & 5.82 & 5.73 & 5.71 & 1.54 & 1.55 & 1.56 & 1.52 & 1.52 \\
\hline BA-29 & $\mathrm{L}$ retrosplenial cingular ctx & -78.9 & 5.96 & 5.74 & 5.66 & 5.64 & 5.59 & 1.87 & 1.92 & 1.96 & 1.96 & 2.12 \\
\hline BA-29 & $\mathrm{R}$ retrosplenial cingular ctx & -81.3 & 5.96 & 5.72 & 5.68 & 5.62 & 5.60 & 1.83 & 1.93 & 1.90 & 1.98 & 2.03 \\
\hline BA-30 & $L$ part of cingular ctx & -69.7 & 6.01 & 5.73 & 5.70 & 5.63 & 5.59 & 1.80 & 1.86 & 1.86 & 1.90 & 1.97 \\
\hline BA-30 & $\mathrm{R}$ part of cingular ctx & -71.4 & 5.91 & 5.61 & 5.59 & 5.54 & 5.52 & 1.58 & 1.66 & 1.62 & 1.66 & 1.67 \\
\hline BA-33 & $\mathrm{L}$ part of ant. cingulate gyrus & -70.9 & 6.18 & 6.00 & 5.97 & 5.92 & 5.90 & 2.12 & 2.20 & 2.20 & 2.23 & 2.33 \\
\hline BA-33 & $\mathrm{R}$ part of ant. cingulate gyrus & -64.8 & 6.28 & 6.07 & 5.99 & 5.95 & 5.97 & 1.64 & 1.71 & 1.73 & 1.75 & 1.79 \\
\hline BA-34 & $\mathrm{L}$ ant. entorhinal ctx & -89.6 & 6.17 & 5.98 & 5.90 & 5.82 & 5.83 & 2.36 & 2.42 & 2.40 & 2.41 & 2.42 \\
\hline BA-34 & $\mathrm{R}$ ant. entorhinal ctx & -87.0 & 6.09 & 5.79 & 5.74 & 5.68 & 5.69 & 1.52 & 1.51 & 1.56 & 1.49 & 1.42 \\
\hline BA-35 & $L$ perirhinal ctx & -90.0 & 6.04 & 5.83 & 5.79 & 5.78 & 5.74 & 2.22 & 2.31 & 2.32 & 2.27 & 2.32 \\
\hline BA-35 & $\mathrm{R}$ perirhinal ctx & -83.8 & 5.99 & 5.76 & 5.72 & 5.69 & 5.68 & 1.55 & 1.52 & 1.53 & 1.52 & 1.60 \\
\hline BA-36 & L parahippocampal ctx & -73.5 & 6.03 & 5.86 & 5.82 & 5.78 & 5.75 & 2.32 & 2.36 & 2.38 & 2.37 & 2.40 \\
\hline BA-36 & $\mathrm{R}$ parahippocampal ctx & -62.5 & 6.04 & 5.80 & 5.73 & 5.72 & 5.71 & 1.65 & 1.66 & 1.68 & 1.61 & 1.69 \\
\hline BA-37 & $L$ fusiform gyrus & -37.4 & 5.99 & 5.79 & 5.76 & 5.75 & 5.69 & 2.22 & 2.27 & 2.23 & 2.27 & 2.39 \\
\hline BA-37 & $\mathrm{R}$ fusiform gyrus & -33.4 & 5.91 & 5.66 & 5.61 & 5.60 & 5.50 & 1.55 & 1.58 & 1.59 & 1.62 & 1.64 \\
\hline BA-38 & $\mathrm{L}$ temporopolar area & -58.3 & 6.19 & 6.02 & 5.93 & 5.89 & 5.90 & 2.29 & 2.39 & 2.40 & 2.37 & 2.44 \\
\hline BA-38 & $\mathrm{R}$ temporopolar area & -53.0 & 6.13 & 5.86 & 5.86 & 5.77 & 5.74 & 1.52 & 1.50 & 1.50 & 1.47 & 1.54 \\
\hline BA-39 & $\mathrm{L}$ angular gyrus & -36.4 & 5.97 & 5.73 & 5.66 & 5.67 & 5.60 & 2.09 & 2.06 & 2.07 & 2.14 & 2.20 \\
\hline BA-39 & $\mathrm{R}$ angular gyrus & -35.3 & 5.95 & 5.72 & 5.65 & 5.66 & 5.61 & 1.69 & 1.81 & 1.81 & 1.86 & 1.96 \\
\hline BA-40 & L supramarginal gyrus & -32.5 & 6.03 & 5.82 & 5.78 & 5.75 & 5.74 & 2.25 & 2.31 & 2.35 & 2.30 & 2.40 \\
\hline BA-40 & R supramarginal gyrus & -30.6 & 6.04 & 5.84 & 5.79 & 5.76 & 5.73 & 1.62 & 1.71 & 1.72 & 1.75 & 1.83 \\
\hline BA-42 & L prim. and auditory assoc. ctx & -47.9 & 6.04 & 5.87 & 5.83 & 5.82 & 5.82 & 2.60 & 2.71 & 2.71 & 2.70 & 2.67 \\
\hline BA-42 & R prim. and auditory assoc. ctx & -41.0 & 6.08 & 5.82 & 5.77 & 5.69 & 5.67 & 1.52 & 1.61 & 1.55 & 1.61 & 1.64 \\
\hline BA-43 & $\mathrm{L}$ subcentral area & -37.7 & 6.15 & 5.90 & 5.94 & 5.87 & 5.89 & 2.49 & 2.54 & 2.61 & 2.55 & 2.58 \\
\hline BA-43 & $\mathrm{R}$ subcentral area & -38.6 & 6.11 & 5.86 & 5.85 & 5.77 & 5.76 & 1.71 & 1.78 & 1.82 & 1.83 & 1.83 \\
\hline
\end{tabular}


Table 7 (Continued).

\begin{tabular}{|c|c|c|c|c|c|c|c|c|c|c|c|c|}
\hline \multirow[b]{2}{*}{ BA } & \multirow[b]{2}{*}{ Region } & \multirow[b]{2}{*}{ Sens. } & \multicolumn{5}{|c|}{$\mathrm{DPF}(\mathrm{nm})$} & \multicolumn{5}{|c|}{$\operatorname{PPF}(\mathrm{nm})$} \\
\hline & & & 690 & 780 & 808 & 830 & 850 & 690 & 780 & 808 & 830 & 850 \\
\hline BA-44 & $\mathrm{L}$ pars opercularis & -42.4 & 6.19 & 6.05 & 6.01 & 6.00 & 5.96 & 2.38 & 2.51 & 2.55 & 2.41 & 2.58 \\
\hline BA-44 & R pars opercularis & -43.5 & 6.23 & 6.04 & 5.97 & 5.96 & 5.85 & 1.61 & 1.53 & 1.57 & 1.74 & 1.71 \\
\hline BA-45 & $\mathrm{L}$ pars triangularis & -39.2 & 6.14 & 5.97 & 5.97 & 5.90 & 5.89 & 2.41 & 2.54 & 2.59 & 2.49 & 2.57 \\
\hline BA-45 & $\mathrm{R}$ pars triangularis & -39.4 & 6.33 & 6.13 & 6.04 & 5.99 & 5.91 & 1.89 & 1.91 & 1.86 & 1.93 & 1.96 \\
\hline BA-46 & $\mathrm{L}$ dorsolateral prefrontal ctx & -36.2 & 6.12 & 5.87 & 5.83 & 5.79 & 5.79 & 2.29 & 2.34 & 2.37 & 2.40 & 2.42 \\
\hline BA-46 & $\mathrm{R}$ dorsolateral prefrontal ctx & -35.8 & 6.35 & 6.11 & 5.99 & 5.96 & 5.87 & 1.41 & 1.45 & 1.47 & 1.52 & 1.57 \\
\hline BA-47 & $L$ inferior prefrontal gyrus & -44.9 & 6.11 & 5.89 & 5.84 & 5.80 & 5.81 & 2.30 & 2.35 & 2.38 & 2.38 & 2.44 \\
\hline BA-47 & $\mathrm{R}$ inferior prefrontal gyrus & -49.1 & 6.26 & 6.01 & 5.94 & 5.87 & 5.91 & 1.51 & 1.51 & 1.52 & 1.53 & 1.59 \\
\hline
\end{tabular}

Note: This table shows the DPF and PPF for several fNIRS-accessible Brodmann areas in the head in female subjects. The sensitivity of the region to fNIRS is provided in decibels $(\mathrm{dB} ; 10 \times \log 10)$ and indicates the fraction of photons normalized to the incidence power reaching this region from the scalp at a source-detector distance of $30 \mathrm{~mm}$.

Table 8 Sensitivity, DPF, PPF of Brodmann areas (males).

\begin{tabular}{|c|c|c|c|c|c|c|c|c|c|c|c|c|}
\hline \multirow[b]{2}{*}{ BA } & \multirow[b]{2}{*}{ Region } & \multirow[b]{2}{*}{ Sens. } & \multicolumn{5}{|c|}{ DPF (nm) } & \multicolumn{5}{|c|}{ PPF (nm) } \\
\hline & & & 690 & 780 & 808 & 830 & 850 & 690 & 780 & 808 & 830 & 850 \\
\hline $\mathrm{BA}-1$ & L prim. somatosensory ctx & -41.6 & 6.18 & 6.03 & 5.95 & 5.93 & 5.91 & 2.73 & 2.81 & 2.82 & 2.79 & 2.85 \\
\hline $\mathrm{BA}-1$ & R prim. somatosensory ctx & -36.6 & 6.15 & 5.93 & 5.89 & 5.79 & 5.80 & 2.15 & 2.14 & 2.08 & 2.19 & 2.23 \\
\hline BA-2 & L prim. somatosensory ctx & -38.0 & 6.16 & 5.97 & 5.91 & 5.92 & 5.86 & 2.70 & 2.76 & 2.78 & 2.80 & 2.80 \\
\hline BA-2 & R prim. somatosensory ctx & -36.5 & 6.14 & 5.88 & 5.79 & 5.75 & 5.74 & 2.13 & 2.15 & 2.10 & 2.18 & 2.20 \\
\hline BA-3 & L prim. somatosensory ctx & -39.1 & 6.13 & 6.01 & 5.99 & 5.98 & 5.93 & 2.74 & 2.80 & 2.84 & 2.80 & 2.82 \\
\hline BA-3 & R prim. somatosensory ctx & -34.5 & 6.13 & 5.89 & 5.83 & 5.78 & 5.76 & 2.14 & 2.19 & 2.21 & 2.19 & 2.23 \\
\hline BA4 & L prim. motor ctx & -39.8 & 6.15 & 6.05 & 6.04 & 6.03 & 6.00 & 2.71 & 2.78 & 2.83 & 2.75 & 2.85 \\
\hline BA-4 & R prim. motor ctx & -40.9 & 6.09 & 5.85 & 5.81 & 5.76 & 5.73 & 2.21 & 2.24 & 2.23 & 2.18 & 2.24 \\
\hline BA-5 & L somatosensory assoc. ctx & -39.8 & 6.07 & 5.88 & 5.89 & 5.85 & 5.85 & 2.63 & 2.66 & 2.70 & 2.68 & 2.71 \\
\hline BA-5 & R somatosensory assoc. ctx & -44.8 & 6.10 & 5.87 & 5.84 & 5.79 & 5.78 & 2.08 & 2.16 & 2.15 & 2.17 & 2.19 \\
\hline BA-6 & $\mathrm{L}$ motor and suppl. motor ctx & -32.1 & 6.18 & 6.03 & 6.01 & 5.99 & 5.96 & 2.71 & 2.79 & 2.79 & 2.82 & 2.80 \\
\hline BA-6 & $\mathrm{R}$ motor and suppl.motor ctx & -28.9 & 6.15 & 5.92 & 5.92 & 5.84 & 5.81 & 2.11 & 2.14 & 2.17 & 2.18 & 2.18 \\
\hline BA-7 & L somatosensory assoc. ctx & -35.4 & 6.06 & 5.91 & 5.85 & 5.82 & 5.80 & 2.66 & 2.71 & 2.72 & 2.73 & 2.77 \\
\hline BA-7 & R somatosensory assoc. ctx & -36.9 & 6.08 & 5.86 & 5.80 & 5.75 & 5.74 & 2.16 & 2.21 & 2.23 & 2.18 & 2.20 \\
\hline BA-8 & $L$ includes frontal eye fields & -34.4 & 6.22 & 6.09 & 6.00 & 5.99 & 5.95 & 2.56 & 2.65 & 2.61 & 2.53 & 2.56 \\
\hline BA-8 & $\mathrm{R}$ includes frontal eye fields & -35.9 & 6.26 & 6.07 & 6.05 & 5.97 & 5.97 & 2.10 & 2.08 & 2.06 & 2.09 & 2.14 \\
\hline BA-9 & $\mathrm{L}$ dorsolateral prefrontal ctx & -34.8 & 6.21 & 6.06 & 6.01 & 6.00 & 5.98 & 2.64 & 2.58 & 2.58 & 2.53 & 2.58 \\
\hline BA-9 & $\mathrm{R}$ dorsolateral prefrontal ctx & -36.3 & 6.29 & 6.04 & 5.97 & 5.94 & 5.90 & 1.97 & 1.96 & 2.01 & 1.95 & 2.02 \\
\hline BA-10 & $L$ frontopolar area & -30.0 & 6.06 & 5.88 & 5.87 & 5.84 & 5.84 & 2.18 & 2.19 & 2.23 & 2.19 & 2.23 \\
\hline BA-10 & $\mathrm{R}$ frontopolar area & -30.5 & 6.23 & 5.95 & 5.89 & 5.84 & 5.79 & 1.61 & 1.61 & 1.62 & 1.63 & 1.63 \\
\hline BA-11 & $\mathrm{L}$ orbitofrontal area & -49.2 & 6.10 & 5.88 & 5.86 & 5.80 & 5.75 & 1.91 & 1.94 & 1.90 & 1.84 & 1.85 \\
\hline
\end{tabular}


Table 8 (Continued).

\begin{tabular}{|c|c|c|c|c|c|c|c|c|c|c|c|c|}
\hline \multirow[b]{2}{*}{ BA } & \multirow[b]{2}{*}{ Region } & \multirow[b]{2}{*}{ Sens. } & \multicolumn{5}{|c|}{$\mathrm{DPF}(\mathrm{nm})$} & \multicolumn{5}{|c|}{$\operatorname{PPF}(n m)$} \\
\hline & & & 690 & 780 & 808 & 830 & 850 & 690 & 780 & 808 & 830 & 850 \\
\hline $\mathrm{BA}-11$ & $\mathrm{R}$ orbitofrontal area & -48.5 & 6.24 & 5.98 & 5.93 & 5.91 & 5.84 & 1.32 & 1.31 & 1.32 & 1.38 & 1.39 \\
\hline BA-17 & L prim. visual ctx (V1) & -38.9 & 5.98 & 5.75 & 5.78 & 5.77 & 5.72 & 2.42 & 2.41 & 2.40 & 2.38 & 2.42 \\
\hline BA-17 & $\mathrm{R}$ prim. visual ctx (V1) & -40.2 & 5.96 & 5.72 & 5.68 & 5.63 & 5.60 & 2.15 & 2.11 & 2.08 & 2.01 & 2.03 \\
\hline BA-18 & L visual assoc. ctx (V2) & -28.7 & 6.02 & 5.81 & 5.79 & 5.73 & 5.71 & 2.57 & 2.54 & 2.57 & 2.50 & 2.53 \\
\hline BA-18 & $\mathrm{R}$ visual assoc. ctx (V2) & -28.0 & 5.93 & 5.63 & 5.61 & 5.54 & 5.51 & 2.09 & 2.14 & 2.16 & 2.15 & 2.18 \\
\hline BA-19 & $\mathrm{L}$ associative visual ctx (V3) & -30.1 & 5.97 & 5.79 & 5.75 & 5.72 & 5.70 & 2.67 & 2.66 & 2.70 & 2.74 & 2.74 \\
\hline BA-19 & $\mathrm{R}$ associative visual ctx (V3) & -31.2 & 5.92 & 5.68 & 5.64 & 5.63 & 5.59 & 2.25 & 2.30 & 2.28 & 2.33 & 2.36 \\
\hline BA-20 & $\mathrm{L}$ inferior temporal gyrus & -45.0 & 6.09 & 5.92 & 5.90 & 5.84 & 5.88 & 2.76 & 2.78 & 2.81 & 2.79 & 2.80 \\
\hline BA-20 & R inferior temporal gyrus & -50.4 & 5.99 & 5.78 & 5.71 & 5.66 & 5.65 & 1.82 & 1.86 & 1.86 & 1.88 & 1.88 \\
\hline BA-21 & $\mathrm{L}$ middle temporal gyrus & -36.7 & 6.08 & 5.90 & 5.88 & 5.84 & 5.81 & 2.62 & 2.68 & 2.75 & 2.63 & 2.63 \\
\hline BA-21 & $\mathrm{R}$ middle temporal gyrus & -36.5 & 6.00 & 5.76 & 5.73 & 5.68 & 5.63 & 1.58 & 1.61 & 1.60 & 1.67 & 1.64 \\
\hline BA-22 & $\mathrm{L}$ superior temporal gyrus & -38.6 & 6.09 & 5.99 & 5.94 & 5.90 & 5.90 & 2.73 & 2.78 & 2.80 & 2.84 & 2.87 \\
\hline BA-22 & R superior temporal gyrus & -36.1 & 6.06 & 5.86 & 5.79 & 5.80 & 5.75 & 1.70 & 1.73 & 1.76 & 1.78 & 1.75 \\
\hline BA-23 & $\mathrm{L}$ ventral post. cingulate $\mathrm{ctx}$ & -86.4 & 6.12 & 5.96 & 5.97 & 5.91 & 5.89 & 2.46 & 2.38 & 2.52 & 2.38 & 2.38 \\
\hline BA-23 & $\mathrm{R}$ ventral post. cingulate $\mathrm{ctx}$ & -81.6 & 5.97 & 5.85 & 5.78 & 5.79 & 5.75 & 1.97 & 1.97 & 1.97 & 2.01 & 2.01 \\
\hline BA-24 & $\mathrm{L}$ ventral ant. cingulate ctx & -74.9 & 6.18 & 5.98 & 5.89 & 5.94 & 5.87 & 2.52 & 2.51 & 2.57 & 2.51 & 2.56 \\
\hline BA-24 & $\mathrm{R}$ ventral ant. cingulate ctx & -75.4 & 6.05 & 5.85 & 5.81 & 5.79 & 5.82 & 2.05 & 2.03 & 2.09 & 2.14 & 2.14 \\
\hline BA-25 & $\mathrm{L}$ subgenual ctx & -77.0 & 6.20 & 5.98 & 5.94 & 5.91 & 5.87 & 2.03 & 2.09 & 2.07 & 2.07 & 2.10 \\
\hline BA-25 & $\mathrm{R}$ subgenual ctx & -64.1 & 6.25 & 6.01 & 5.95 & 5.93 & 5.90 & 2.06 & 2.08 & 2.11 & 2.15 & 2.15 \\
\hline BA-28 & L post. entorhinal CTx & -85.4 & 6.22 & 6.07 & 6.02 & 6.00 & 5.98 & 2.81 & 2.93 & 3.04 & 2.94 & 2.94 \\
\hline BA-28 & $\mathrm{R}$ post. entorhinal ctx & -86.1 & 6.02 & 5.76 & 5.76 & 5.70 & 5.71 & 1.90 & 1.89 & 1.87 & 2.01 & 1.98 \\
\hline BA-29 & $\mathrm{L}$ retrosplenial cingular ctx & -79.4 & 6.02 & 5.82 & 5.79 & 5.75 & 5.71 & 2.67 & 2.66 & 2.66 & 2.63 & 2.63 \\
\hline BA-29 & $\mathrm{R}$ retrosplenial cingular ctx & -81.2 & 6.00 & 5.83 & 5.78 & 5.74 & 5.77 & 2.46 & 2.42 & 2.42 & 2.40 & 2.42 \\
\hline BA-30 & $\mathrm{L}$ part of cingular ctx & -70.0 & 6.00 & 5.84 & 5.77 & 5.73 & 5.70 & 2.79 & 2.78 & 2.81 & 2.86 & 2.83 \\
\hline BA-30 & $\mathrm{R}$ part of cingular ctx & -70.5 & 5.93 & 5.64 & 5.60 & 5.55 & 5.52 & 2.16 & 2.11 & 2.14 & 2.24 & 2.25 \\
\hline BA-33 & $\mathrm{L}$ part of ant. cingulate gyrus & -72.5 & 6.25 & 6.06 & 6.06 & 6.00 & 5.97 & 2.54 & 2.55 & 2.64 & 2.59 & 2.57 \\
\hline BA-33 & $\mathrm{R}$ part of ant. cingulate gyrus & -62.3 & 6.22 & 6.06 & 6.05 & 6.02 & 5.99 & 2.08 & 2.12 & 2.12 & 2.09 & 2.10 \\
\hline BA-34 & $L$ ant. entorhinal ctx & -90.4 & 6.12 & 6.06 & 5.98 & 5.95 & 5.91 & 2.66 & 2.73 & 2.78 & 2.80 & 2.76 \\
\hline BA-34 & $R$ ant. entorhinal ctx & -87.6 & 6.10 & 5.81 & 5.77 & 5.74 & 5.72 & 2.04 & 2.03 & 2.00 & 2.06 & 2.08 \\
\hline BA-35 & $\mathrm{L}$ perirhinal ctx & -90.6 & 6.02 & 5.84 & 5.80 & 5.76 & 5.76 & 2.58 & 2.64 & 2.67 & 2.62 & 2.67 \\
\hline BA-35 & $\mathrm{R}$ perirhinal ctx & -86.6 & 6.00 & 5.78 & 5.72 & 5.69 & 5.67 & 1.64 & 1.58 & 1.59 & 1.66 & 1.67 \\
\hline BA-36 & L parahippocampal ctx & -76.5 & 6.06 & 5.86 & 5.82 & 5.81 & 5.79 & 2.65 & 2.74 & 2.75 & 2.68 & 2.70 \\
\hline BA-36 & R parahippocampal ctx & -67.6 & 5.96 & 5.74 & 5.71 & 5.67 & 5.64 & 1.70 & 1.73 & 1.71 & 1.71 & 1.73 \\
\hline BA-37 & $L$ fusiform gyrus & -37.3 & 5.93 & 5.76 & 5.69 & 5.61 & 5.61 & 2.41 & 2.43 & 2.45 & 2.51 & 2.52 \\
\hline BA-37 & $\mathrm{R}$ fusiform gyrus & -35.2 & 5.99 & 5.73 & 5.71 & 5.66 & 5.61 & 1.83 & 1.91 & 1.93 & 1.95 & 2.00 \\
\hline BA-38 & $\mathrm{L}$ temporopolar area & -55.9 & 6.22 & 6.00 & 5.99 & 5.99 & 5.91 & 2.69 & 2.80 & 2.85 & 2.86 & 2.86 \\
\hline BA-38 & $\mathrm{R}$ temporopolar area & -52.8 & 6.08 & 5.91 & 5.88 & 5.81 & 5.77 & 1.84 & 1.88 & 1.86 & 1.93 & 1.92 \\
\hline BA-39 & $\mathrm{L}$ angular gyrus & -35.2 & 5.96 & 5.80 & 5.79 & 5.75 & 5.72 & 2.58 & 2.63 & 2.68 & 2.65 & 2.70 \\
\hline
\end{tabular}


Table 8 (Continued).

\begin{tabular}{|c|c|c|c|c|c|c|c|c|c|c|c|c|}
\hline \multirow[b]{2}{*}{ BA } & \multirow[b]{2}{*}{ Region } & \multirow[b]{2}{*}{ Sens. } & \multicolumn{5}{|c|}{ DPF (nm) } & \multicolumn{5}{|c|}{$\operatorname{PPF}(\mathrm{nm})$} \\
\hline & & & 690 & 780 & 808 & 830 & 850 & 690 & 780 & 808 & 830 & 850 \\
\hline BA-39 & $\mathrm{R}$ angular gyrus & -34.7 & 5.98 & 5.72 & 5.64 & 5.63 & 5.59 & 2.01 & 2.06 & 2.04 & 2.09 & 2.06 \\
\hline $\mathrm{BA}-40$ & L supramarginal gyrus & -33.4 & 6.05 & 5.88 & 5.84 & 5.84 & 5.85 & 2.69 & 2.68 & 2.72 & 2.80 & 2.77 \\
\hline BA-40 & R supramarginal gyrus & -31.5 & 6.02 & 5.82 & 5.75 & 5.73 & 5.71 & 1.79 & 1.78 & 1.79 & 1.87 & 1.90 \\
\hline BA-42 & L prim. and auditory assoc. ctx & -45.2 & 6.03 & 5.92 & 5.89 & 5.89 & 5.82 & 2.75 & 2.80 & 2.78 & 2.86 & 2.83 \\
\hline $\mathrm{BA}-42$ & R prim. and auditory assoc. ctx & -38.5 & 6.05 & 5.82 & 5.79 & 5.83 & 5.77 & 1.75 & 1.81 & 1.80 & 1.91 & 1.79 \\
\hline $\mathrm{BA}-43$ & $\mathrm{~L}$ subcentral area & -37.6 & 6.13 & 6.07 & 6.00 & 6.07 & 6.00 & 2.95 & 2.99 & 2.98 & 3.09 & 3.06 \\
\hline BA-43 & $\mathrm{R}$ subcentral area & -41.2 & 6.04 & 5.80 & 5.82 & 5.84 & 5.74 & 1.78 & 1.80 & 1.86 & 1.86 & 1.86 \\
\hline $\mathrm{BA}-44$ & $\mathrm{~L}$ pars opercularis & -43.7 & 6.14 & 6.07 & 5.98 & 6.00 & 5.99 & 2.81 & 2.83 & 2.85 & 2.90 & 2.93 \\
\hline $\mathrm{BA}-44$ & R pars opercularis & -44.0 & 6.20 & 5.95 & 5.92 & 5.85 & 5.83 & 1.65 & 1.65 & 1.68 & 1.69 & 1.73 \\
\hline BA-45 & $\mathrm{L}$ pars triangularis & -40.3 & 6.15 & 6.00 & 5.94 & 5.91 & 5.93 & 2.67 & 2.75 & 2.75 & 2.74 & 2.76 \\
\hline BA-45 & $\mathrm{R}$ pars triangularis & -39.5 & 6.27 & 6.01 & 5.97 & 5.92 & 5.94 & 1.72 & 1.66 & 1.80 & 1.77 & 1.76 \\
\hline BA-46 & $\mathrm{L}$ dorsolateral prefrontal ctx & -36.4 & 6.20 & 6.04 & 5.99 & 5.92 & 5.93 & 2.62 & 2.68 & 2.68 & 2.66 & 2.69 \\
\hline BA-46 & $\mathrm{R}$ dorsolateral prefrontal ctx & -37.4 & 6.27 & 6.00 & 5.95 & 5.94 & 5.89 & 1.72 & 1.76 & 1.75 & 1.78 & 1.83 \\
\hline BA-47 & L inferior prefrontal gyrus & -48.6 & 6.12 & 5.97 & 5.89 & 5.88 & 5.86 & 2.47 & 2.50 & 2.58 & 2.57 & 2.57 \\
\hline BA-47 & $\mathrm{R}$ inferior prefrontal gyrus & -47.4 & 6.30 & 6.00 & 5.96 & 5.92 & 5.88 & 1.91 & 1.95 & 1.99 & 2.01 & 2.02 \\
\hline
\end{tabular}

Table 9 Sensitivity, DPF, PPF of anatomical regions (females).

\begin{tabular}{|c|c|c|c|c|c|c|c|c|c|c|c|}
\hline \multirow[b]{2}{*}{ Region } & \multirow[b]{2}{*}{ Sens. } & \multicolumn{5}{|c|}{$\mathrm{DPF}(\mathrm{nm})$} & \multicolumn{5}{|c|}{$\operatorname{PPF}(\mathrm{nm})$} \\
\hline & & 690 & 780 & 808 & 830 & 850 & 690 & 780 & 808 & 830 & 850 \\
\hline$L$ banks of the superior temporal sulcus & -46.4 & 5.97 & 5.76 & 5.66 & 5.66 & 5.65 & 2.26 & 2.28 & 2.30 & 2.26 & 2.37 \\
\hline $\mathrm{R}$ banks of the superior temporal sulcus & -45.8 & 6.03 & 5.69 & 5.75 & 5.69 & 5.65 & 1.62 & 1.61 & 1.55 & 1.65 & 1.76 \\
\hline L caudal anterior cingulate & -79.0 & 6.18 & 6.00 & 5.94 & 5.92 & 5.88 & 2.12 & 2.18 & 2.21 & 2.21 & 2.43 \\
\hline $\mathrm{R}$ caudal anterior cingulate & -78.8 & 6.17 & 6.05 & 5.99 & 5.95 & 5.92 & 1.83 & 1.94 & 1.97 & 2.03 & 2.22 \\
\hline L caudal middle frontal & -37.3 & 6.14 & 5.94 & 5.91 & 5.92 & 5.90 & 2.40 & 2.44 & 2.49 & 2.48 & 2.54 \\
\hline $\mathrm{R}$ caudal middle frontal & -38.0 & 6.21 & 6.03 & 5.97 & 5.93 & 5.93 & 1.89 & 1.93 & 1.93 & 1.97 & 1.99 \\
\hline$L$ cuneus & -52.7 & 5.98 & 5.71 & 5.60 & 5.55 & 5.52 & 1.59 & 1.80 & 1.81 & 2.00 & 2.00 \\
\hline$R$ cuneus & -48.7 & 5.95 & 5.67 & 5.62 & 5.55 & 5.58 & 1.43 & 1.48 & 1.45 & 1.51 & 1.50 \\
\hline L entorhinal & -90.5 & 6.05 & 5.95 & 5.88 & 5.85 & 5.83 & 2.23 & 2.38 & 2.31 & 2.39 & 2.40 \\
\hline R entorhinal & -89.9 & 6.02 & 5.79 & 5.78 & 5.65 & 5.61 & 1.54 & 1.53 & 1.54 & 1.54 & 1.57 \\
\hline$L$ frontal pole & -38.5 & 5.97 & 5.70 & 5.68 & 5.61 & 5.56 & 1.38 & 1.38 & 1.36 & 1.42 & 1.65 \\
\hline$R$ frontal pole & -40.1 & 6.23 & 5.89 & 5.81 & 5.79 & 5.72 & 1.18 & 1.13 & 1.12 & 1.19 & 1.21 \\
\hline$L$ fusiform & -55.6 & 6.04 & 5.84 & 5.76 & 5.73 & 5.69 & 2.27 & 2.34 & 2.32 & 2.35 & 2.37 \\
\hline
\end{tabular}


Table 9 (Continued).

\begin{tabular}{|c|c|c|c|c|c|c|c|c|c|c|c|}
\hline \multirow[b]{2}{*}{ Region } & \multirow[b]{2}{*}{ Sens. } & \multicolumn{5}{|c|}{$\mathrm{DPF}(\mathrm{nm})$} & \multicolumn{5}{|c|}{$\operatorname{PPF}(n m)$} \\
\hline & & 690 & 780 & 808 & 830 & 850 & 690 & 780 & 808 & 830 & 850 \\
\hline$R$ fusiform & -59.8 & 6.01 & 5.77 & 5.72 & 5.70 & 5.65 & 1.58 & 1.60 & 1.59 & 1.60 & 1.63 \\
\hline$L$ inferior parietal & -29.8 & 5.98 & 5.73 & 5.69 & 5.67 & 5.60 & 2.01 & 2.01 & 2.02 & 2.08 & 2.20 \\
\hline $\mathrm{R}$ inferior parietal & -30.7 & 5.96 & 5.75 & 5.66 & 5.65 & 5.59 & 1.62 & 1.77 & 1.76 & 1.81 & 1.96 \\
\hline$L$ inferior temporal & -35.1 & 6.03 & 5.85 & 5.80 & 5.79 & 5.74 & 2.30 & 2.43 & 2.42 & 2.39 & 2.46 \\
\hline $\mathrm{R}$ inferior temporal & -34.6 & 6.03 & 5.73 & 5.68 & 5.65 & 5.57 & 1.56 & 1.56 & 1.55 & 1.53 & 1.61 \\
\hline$L$ insula & -62.0 & 6.15 & 5.98 & 5.94 & 5.91 & 5.91 & 2.47 & 2.55 & 2.59 & 2.57 & 2.61 \\
\hline$R$ insula & -61.1 & 6.19 & 5.91 & 5.91 & 5.80 & 5.76 & 1.63 & 1.62 & 1.61 & 1.66 & 1.72 \\
\hline $\mathrm{L}$ isthmus cingulate & -88.0 & 5.96 & 5.77 & 5.73 & 5.65 & 5.60 & 2.04 & 2.07 & 2.06 & 2.09 & 2.16 \\
\hline$R$ isthmus cingulate & -90.1 & 5.96 & 5.69 & 5.65 & 5.63 & 5.56 & 1.89 & 1.91 & 1.93 & 1.94 & 1.94 \\
\hline L lateral occipital & -27.7 & 6.02 & 5.74 & 5.69 & 5.64 & 5.61 & 1.79 & 1.84 & 1.85 & 1.84 & 1.92 \\
\hline R lateral occipital & -27.9 & 5.97 & 5.67 & 5.59 & 5.54 & 5.51 & 1.25 & 1.31 & 1.33 & 1.37 & 1.40 \\
\hline L lateral orbitofrontal & -64.1 & 6.01 & 5.83 & 5.82 & 5.76 & 5.70 & 2.03 & 2.07 & 2.15 & 2.11 & 2.15 \\
\hline R lateral orbitofrontal & -62.8 & 6.27 & 5.93 & 5.90 & 5.85 & 5.84 & 1.30 & 1.24 & 1.25 & 1.30 & 1.35 \\
\hline$L$ lingual & -57.6 & 6.06 & 5.76 & 5.70 & 5.64 & 5.61 & 1.67 & 1.70 & 1.70 & 1.75 & 1.90 \\
\hline $\mathrm{R}$ lingual & -71.8 & 5.97 & 5.62 & 5.59 & 5.52 & 5.47 & 1.24 & 1.31 & 1.29 & 1.38 & 1.51 \\
\hline L medial orbitofrontal & -64.2 & 5.96 & 5.73 & 5.67 & 5.63 & 5.60 & 1.49 & 1.43 & 1.44 & 1.53 & 1.55 \\
\hline R medial orbitofrontal & -61.5 & 6.21 & 5.93 & 5.88 & 5.78 & 5.74 & 1.13 & 1.11 & 1.10 & 1.10 & 1.23 \\
\hline $\mathrm{L}$ middle temporal & -28.9 & 6.04 & 5.86 & 5.83 & 5.81 & 5.77 & 2.38 & 2.38 & 2.38 & 2.39 & 2.46 \\
\hline $\mathrm{R}$ middle temporal & -26.9 & 6.04 & 5.77 & 5.71 & 5.68 & 5.66 & 1.68 & 1.71 & 1.70 & 1.65 & 1.73 \\
\hline L paracentral & -53.3 & 6.06 & 5.88 & 5.89 & 5.83 & 5.80 & 2.35 & 2.35 & 2.39 & 2.47 & 2.51 \\
\hline R paracentral & -50.7 & 6.08 & 5.86 & 5.82 & 5.77 & 5.78 & 1.91 & 1.99 & 2.03 & 2.15 & 2.24 \\
\hline L parahippocampal & -85.4 & 6.02 & 5.83 & 5.77 & 5.73 & 5.74 & 2.22 & 2.32 & 2.26 & 2.38 & 2.47 \\
\hline $\mathrm{R}$ parahippocampal & -84.3 & 6.01 & 5.76 & 5.70 & 5.68 & 5.70 & 1.56 & 1.55 & 1.57 & 1.53 & 1.57 \\
\hline L pars opercularis & -42.8 & 6.12 & 5.97 & 5.94 & 5.90 & 5.89 & 2.42 & 2.56 & 2.59 & 2.53 & 2.61 \\
\hline $\mathrm{R}$ pars opercularis & -44.4 & 6.34 & 6.12 & 6.02 & 6.00 & 5.91 & 1.67 & 1.74 & 1.70 & 1.75 & 1.79 \\
\hline L pars orbitalis & -42.4 & 6.06 & 5.86 & 5.91 & 5.79 & 5.82 & 2.45 & 2.49 & 2.54 & 2.51 & 2.59 \\
\hline $\mathrm{R}$ pars orbitalis & -40.1 & 6.31 & 5.97 & 5.94 & 5.91 & 5.86 & 1.37 & 1.36 & 1.34 & 1.39 & 1.40 \\
\hline $\mathrm{L}$ pars triangularis & -36.9 & 6.17 & 5.97 & 5.93 & 5.85 & 5.89 & 2.36 & 2.43 & 2.47 & 2.46 & 2.50 \\
\hline $\mathrm{R}$ pars triangularis & -36.1 & 6.32 & 6.13 & 6.07 & 6.00 & 5.97 & 1.68 & 1.67 & 1.65 & 1.70 & 1.81 \\
\hline$L$ pericalcarine & -45.5 & 6.00 & 5.71 & 5.66 & 5.62 & 5.57 & 1.83 & 1.89 & 1.86 & 1.94 & 1.96 \\
\hline $\mathrm{R}$ pericalcarine & -47.0 & 5.97 & 5.69 & 5.65 & 5.57 & 5.57 & 1.46 & 1.49 & 1.52 & 1.56 & 1.58 \\
\hline L postcentral & -30.8 & 6.10 & 5.90 & 5.89 & 5.84 & 5.83 & 2.29 & 2.37 & 2.37 & 2.34 & 2.42 \\
\hline $\mathrm{R}$ postcentral & -29.7 & 6.04 & 5.85 & 5.84 & 5.78 & 5.76 & 1.69 & 1.83 & 1.83 & 1.84 & 1.86 \\
\hline
\end{tabular}


Table 9 (Continued).

\begin{tabular}{|c|c|c|c|c|c|c|c|c|c|c|c|}
\hline \multirow[b]{2}{*}{ Region } & \multirow[b]{2}{*}{ Sens. } & \multicolumn{5}{|c|}{$\mathrm{DPF}(\mathrm{nm})$} & \multicolumn{5}{|c|}{$\operatorname{PPF}(\mathrm{nm})$} \\
\hline & & 690 & 780 & 808 & 830 & 850 & 690 & 780 & 808 & 830 & 850 \\
\hline L posterior cingulate & -80.6 & 6.15 & 5.94 & 5.96 & 5.92 & 5.88 & 2.39 & 2.40 & 2.39 & 2.53 & 2.69 \\
\hline $\mathrm{R}$ posterior cingulate & -80.1 & 6.14 & 5.92 & 5.86 & 5.85 & 5.84 & 2.07 & 2.21 & 2.21 & 2.19 & 2.27 \\
\hline L precentral & -33.9 & 6.11 & 5.96 & 5.93 & 5.88 & 5.86 & 2.33 & 2.46 & 2.46 & 2.38 & 2.50 \\
\hline R precentral & -31.9 & 6.12 & 5.91 & 5.85 & 5.81 & 5.78 & 1.68 & 1.85 & 1.84 & 1.87 & 1.95 \\
\hline L precuneus & -58.6 & 5.96 & 5.72 & 5.68 & 5.61 & 5.57 & 1.91 & 1.93 & 1.96 & 1.97 & 1.97 \\
\hline $\mathrm{R}$ precuneus & -55.9 & 5.94 & 5.67 & 5.61 & 5.58 & 5.56 & 1.79 & 1.85 & 1.86 & 1.89 & 1.93 \\
\hline $\mathrm{L}$ rostral anterior cingulate & -71.1 & 5.99 & 5.81 & 5.74 & 5.67 & 5.62 & 1.54 & 1.57 & 1.60 & 1.60 & 1.81 \\
\hline$R$ rostral anterior cingulate & -75.1 & 6.18 & 5.87 & 5.79 & 5.75 & 5.68 & 1.44 & 1.37 & 1.38 & 1.48 & 1.55 \\
\hline $\mathrm{L}$ rostral middle frontal & -31.6 & 6.08 & 5.88 & 5.80 & 5.77 & 5.78 & 2.00 & 2.00 & 2.06 & 2.02 & 2.13 \\
\hline $\mathrm{R}$ rostral middle frontal & -32.4 & 6.33 & 6.06 & 5.97 & 5.93 & 5.89 & 1.42 & 1.50 & 1.54 & 1.55 & 1.55 \\
\hline L superior frontal & -33.0 & 6.16 & 5.96 & 5.89 & 5.87 & 5.85 & 2.02 & 2.10 & 2.09 & 2.11 & 2.17 \\
\hline R superior frontal & -34.2 & 6.20 & 6.00 & 5.95 & 5.94 & 5.86 & 1.79 & 1.84 & 1.87 & 1.90 & 1.99 \\
\hline L superior parietal & -37.8 & 5.97 & 5.76 & 5.70 & 5.65 & 5.63 & 2.04 & 2.13 & 2.09 & 2.11 & 2.17 \\
\hline $\mathrm{R}$ superior parietal & -34.6 & 6.01 & 5.73 & 5.69 & 5.66 & 5.61 & 1.81 & 1.85 & 1.87 & 1.88 & 1.96 \\
\hline L superior temporal & -41.5 & 6.09 & 5.91 & 5.87 & 5.84 & 5.83 & 2.42 & 2.51 & 2.51 & 2.52 & 2.56 \\
\hline R superior temporal & -32.8 & 6.08 & 5.86 & 5.84 & 5.76 & 5.75 & 1.64 & 1.67 & 1.66 & 1.67 & 1.73 \\
\hline L supramarginal & -33.8 & 5.98 & 5.81 & 5.74 & 5.71 & 5.69 & 2.18 & 2.22 & 2.21 & 2.25 & 2.28 \\
\hline R supramarginal & -32.0 & 6.07 & 5.87 & 5.83 & 5.79 & 5.74 & 1.59 & 1.66 & 1.66 & 1.73 & 1.86 \\
\hline L temporal pole & -73.1 & 6.13 & 5.93 & 5.89 & 5.81 & 5.86 & 2.27 & 2.40 & 2.37 & 2.34 & 2.33 \\
\hline $\mathrm{R}$ temporal pole & -74.0 & 6.12 & 5.79 & 5.83 & 5.68 & 5.64 & 1.72 & 1.74 & 1.73 & 1.78 & 1.78 \\
\hline $\mathrm{L}$ transverse temporal & -64.9 & 6.08 & 5.91 & 5.86 & 5.83 & 5.84 & 2.76 & 2.71 & 2.71 & 2.84 & 2.80 \\
\hline $\mathrm{R}$ transverse temporal & -55.0 & 6.08 & 5.83 & 5.81 & 5.71 & 5.70 & 1.72 & 1.71 & 1.72 & 1.78 & 1.85 \\
\hline
\end{tabular}

Note: This table shows the DPF and PPF for several fNIRS-accessible anatomical regions in the head in female subjects. The sensitivity of the region to fNIRS is provided in decibels $(\mathrm{dB} ; 10 \times \log 10)$ and indicates the fraction of photons normalized to the incidence power reaching this region from the scalp at a source-detector distance of $30 \mathrm{~mm}$.

Table 10 Sensitivity, DPF, PPF of anatomical regions (males).

\begin{tabular}{|c|c|c|c|c|c|c|c|c|c|c|c|}
\hline \multirow[b]{2}{*}{ Region } & \multirow[b]{2}{*}{ Sens. } & \multicolumn{5}{|c|}{ DPF $(n m)$} & \multicolumn{5}{|c|}{ PPF (nm) } \\
\hline & & 690 & 780 & 808 & 830 & 850 & 690 & 780 & 808 & 830 & 850 \\
\hline$L$ banks of the superior temporal sulcus & -42.7 & 5.96 & 5.76 & 5.73 & 5.72 & 5.72 & 2.31 & 2.37 & 2.39 & 2.48 & 2.51 \\
\hline $\mathrm{R}$ banks of the superior temporal sulcus & -46.2 & 6.05 & 5.74 & 5.70 & 5.69 & 5.61 & 1.74 & 1.78 & 1.80 & 1.78 & 1.81 \\
\hline $\mathrm{L}$ caudal anterior cingulate & -80.1 & 6.26 & 6.04 & 5.99 & 5.96 & 5.90 & 2.52 & 2.52 & 2.53 & 2.47 & 2.50 \\
\hline $\mathrm{R}$ caudal anterior cingulate & -80.4 & 6.24 & 6.03 & 6.05 & 6.01 & 5.97 & 2.14 & 2.10 & 2.16 & 2.12 & 2.16 \\
\hline L caudal middle frontal & -36.3 & 6.17 & 5.99 & 5.97 & 5.98 & 5.97 & 2.80 & 2.86 & 2.89 & 2.80 & 2.83 \\
\hline
\end{tabular}


Table 10 (Continued).

\begin{tabular}{|c|c|c|c|c|c|c|c|c|c|c|c|}
\hline \multirow[b]{2}{*}{ Region } & \multirow[b]{2}{*}{ Sens. } & \multicolumn{5}{|c|}{$\mathrm{DPF}(\mathrm{nm})$} & \multicolumn{5}{|c|}{ PPF (nm) } \\
\hline & & 690 & 780 & 808 & 830 & 850 & 690 & 780 & 808 & 830 & 850 \\
\hline $\mathrm{R}$ caudal middle frontal & -37.4 & 6.22 & 6.05 & 5.97 & 5.95 & 5.88 & 2.06 & 2.06 & 2.05 & 2.11 & 2.17 \\
\hline $\mathrm{L}$ cuneus & -53.3 & 5.97 & 5.77 & 5.70 & 5.68 & 5.65 & 2.60 & 2.64 & 2.67 & 2.59 & 2.66 \\
\hline$R$ cuneus & -47.4 & 5.89 & 5.73 & 5.65 & 5.59 & 5.55 & 2.21 & 2.12 & 2.16 & 2.02 & 2.03 \\
\hline L entorhinal & -90.5 & 6.16 & 5.99 & 5.96 & 5.91 & 5.92 & 2.68 & 2.80 & 2.80 & 2.80 & 2.81 \\
\hline$R$ entorhinal & -91.5 & 6.03 & 5.78 & 5.76 & 5.75 & 5.73 & 2.10 & 2.12 & 2.02 & 2.24 & 2.23 \\
\hline$L$ frontal pole & -38.8 & 6.10 & 5.84 & 5.81 & 5.71 & 5.67 & 1.77 & 1.81 & 1.80 & 1.87 & 1.87 \\
\hline $\mathrm{R}$ frontal pole & -40.5 & 6.19 & 5.96 & 5.85 & 5.86 & 5.78 & 1.58 & 1.61 & 1.59 & 1.65 & 1.65 \\
\hline$L$ fusiform & -51.4 & 6.03 & 5.80 & 5.79 & 5.75 & 5.75 & 2.62 & 2.64 & 2.65 & 2.68 & 2.72 \\
\hline R fusiform & -56.7 & 6.03 & 5.78 & 5.75 & 5.67 & 5.68 & 1.79 & 1.83 & 1.83 & 1.82 & 1.85 \\
\hline $\mathrm{L}$ inferior parietal & -29.6 & 5.98 & 5.79 & 5.77 & 5.73 & 5.70 & 2.61 & 2.60 & 2.61 & 2.65 & 2.65 \\
\hline $\mathrm{R}$ inferior parietal & -32.1 & 6.01 & 5.73 & 5.67 & 5.66 & 5.60 & 1.99 & 2.03 & 2.01 & 2.08 & 2.09 \\
\hline$L$ inferior temporal & -37.7 & 6.05 & 5.86 & 5.78 & 5.76 & 5.75 & 2.56 & 2.44 & 2.46 & 2.48 & 2.49 \\
\hline $\mathrm{R}$ inferior temporal & -35.1 & 6.01 & 5.73 & 5.67 & 5.68 & 5.62 & 1.82 & 1.85 & 1.88 & 1.87 & 1.89 \\
\hline$L$ insula & -2.3 & 6.15 & 6.02 & 5.99 & 5.96 & 5.93 & 2.70 & 2.78 & 2.81 & 2.82 & 2.84 \\
\hline$R$ insula & -63.0 & 6.08 & 5.89 & 5.84 & 5.81 & 5.76 & 1.77 & 1.77 & 1.81 & 1.80 & 1.81 \\
\hline $\mathrm{L}$ isthmus cingulate & -89.7 & 5.99 & 5.81 & 5.74 & 5.73 & 5.74 & 2.60 & 2.59 & 2.62 & 2.62 & 2.56 \\
\hline $\mathrm{R}$ isthmus cingulate & -89.6 & 6.01 & 5.81 & 5.82 & 5.79 & 5.78 & 2.70 & 2.71 & 2.72 & 2.59 & 2.61 \\
\hline L lateral occipital & -27.1 & 6.03 & 5.87 & 5.82 & 5.80 & 5.75 & 2.62 & 2.51 & 2.55 & 2.70 & 2.73 \\
\hline R lateral occipital & -28.6 & 5.95 & 5.73 & 5.69 & 5.64 & 5.59 & 1.99 & 1.98 & 2.01 & 2.05 & 2.05 \\
\hline L lateral orbitofrontal & -60.9 & 6.12 & 5.95 & 5.91 & 5.88 & 5.84 & 2.21 & 2.21 & 2.25 & 2.23 & 2.24 \\
\hline $\mathrm{R}$ lateral orbitofrontal & -63.4 & 6.26 & 6.02 & 5.95 & 5.94 & 5.85 & 1.49 & 1.52 & 1.52 & 1.55 & 1.54 \\
\hline$L$ lingual & -81.1 & 6.02 & 5.85 & 5.82 & 5.79 & 5.81 & 2.62 & 2.56 & 2.59 & 2.70 & 2.70 \\
\hline $\mathrm{R}$ lingual & -70.5 & 5.91 & 5.66 & 5.62 & 5.58 & 5.56 & 1.99 & 2.03 & 2.01 & 2.07 & 2.07 \\
\hline L medial orbitofrontal & -62.5 & 6.03 & 5.81 & 5.78 & 5.78 & 5.70 & 1.58 & 1.54 & 1.52 & 1.57 & 1.53 \\
\hline R medial orbitofrontal & -61.3 & 6.19 & 5.94 & 5.85 & 5.85 & 5.79 & 1.37 & 1.36 & 1.36 & 1.35 & 1.36 \\
\hline $\mathrm{L}$ middle temporal & -28.6 & 6.07 & 5.88 & 5.82 & 5.83 & 5.76 & 2.56 & 2.57 & 2.60 & 2.61 & 2.60 \\
\hline $\mathrm{R}$ middle temporal & -34.1 & 6.01 & 5.79 & 5.72 & 5.72 & 5.64 & 1.71 & 1.75 & 1.72 & 1.79 & 1.77 \\
\hline $\mathrm{L}$ paracentral & -51.9 & 6.09 & 5.98 & 5.99 & 5.93 & 5.90 & 2.64 & 2.67 & 2.69 & 2.69 & 2.72 \\
\hline R paracentral & -49.3 & 6.09 & 5.85 & 5.80 & 5.81 & 5.76 & 2.22 & 2.27 & 2.27 & 2.29 & 2.30 \\
\hline L parahippocampal & -86.8 & 6.03 & 5.85 & 5.77 & 5.76 & 5.75 & 2.64 & 2.64 & 2.67 & 2.60 & 2.61 \\
\hline R parahippocampal & -86.1 & 5.95 & 5.68 & 5.62 & 5.58 & 5.55 & 1.75 & 1.79 & 1.80 & 1.82 & 1.84 \\
\hline $\mathrm{L}$ pars opercularis & -42.7 & 6.15 & 5.94 & 5.90 & 5.90 & 5.87 & 2.60 & 2.74 & 2.74 & 2.69 & 2.76 \\
\hline $\mathrm{R}$ pars opercularis & -46.9 & 6.23 & 5.99 & 5.95 & 5.91 & 5.90 & 1.74 & 1.72 & 1.77 & 1.81 & 1.81 \\
\hline
\end{tabular}


Table 10 (Continued).

\begin{tabular}{|c|c|c|c|c|c|c|c|c|c|c|c|}
\hline \multirow[b]{2}{*}{ Region } & \multirow[b]{2}{*}{ Sens. } & \multicolumn{5}{|c|}{$\mathrm{DPF}(\mathrm{nm})$} & \multicolumn{5}{|c|}{$\mathrm{PPF}(\mathrm{nm})$} \\
\hline & & 690 & 780 & 808 & 830 & 850 & 690 & 780 & 808 & 830 & 850 \\
\hline L pars orbitalis & -39.9 & 6.15 & 5.98 & 5.97 & 6.01 & 5.95 & 2.34 & 2.40 & 2.44 & 2.42 & 2.42 \\
\hline $\mathrm{R}$ pars orbitalis & -38.7 & 6.35 & 6.04 & 6.05 & 5.95 & 5.95 & 1.31 & 1.34 & 1.33 & 1.40 & 1.38 \\
\hline $\mathrm{L}$ pars triangularis & -36.8 & 6.12 & 6.00 & 5.91 & 5.93 & 5.88 & 2.66 & 2.79 & 2.77 & 2.75 & 2.78 \\
\hline $\mathrm{R}$ pars triangularis & -35.5 & 6.25 & 6.02 & 6.04 & 6.03 & 5.94 & 1.93 & 1.98 & 1.98 & 2.03 & 2.03 \\
\hline$L$ pericalcarine & -47.3 & 5.99 & 5.80 & 5.75 & 5.69 & 5.67 & 2.57 & 2.49 & 2.51 & 2.42 & 2.45 \\
\hline $\mathrm{R}$ pericalcarine & -51.0 & 5.94 & 5.74 & 5.68 & 5.66 & 5.63 & 2.30 & 2.32 & 2.35 & 2.31 & 2.31 \\
\hline L postcentral & -30.6 & 6.13 & 6.00 & 5.93 & 5.94 & 5.93 & 2.74 & 2.78 & 2.80 & 2.77 & 2.79 \\
\hline R postcentral & -29.4 & 6.12 & 5.90 & 5.86 & 5.81 & 5.80 & 2.07 & 2.14 & 2.13 & 2.14 & 2.17 \\
\hline L posterior cingulate & -80.1 & 6.13 & 6.01 & 6.00 & 5.98 & 5.93 & 2.65 & 2.74 & 2.76 & 2.68 & 2.70 \\
\hline $\mathrm{R}$ posterior cingulate & -81.5 & 6.08 & 5.86 & 5.82 & 5.82 & 5.80 & 2.28 & 2.31 & 2.37 & 2.33 & 2.33 \\
\hline L precentral & -32.9 & 6.14 & 6.02 & 6.03 & 6.05 & 6.02 & 2.78 & 2.85 & 2.86 & 2.84 & 2.86 \\
\hline R precentral & -32.1 & 6.12 & 5.94 & 5.90 & 5.85 & 5.81 & 2.11 & 2.14 & 2.13 & 2.07 & 2.08 \\
\hline$L$ precuneus & -56.4 & 6.00 & 5.91 & 5.87 & 5.85 & 5.83 & 2.72 & 2.70 & 2.71 & 2.67 & 2.65 \\
\hline $\mathrm{R}$ precuneus & -55.9 & 6.03 & 5.88 & 5.85 & 5.80 & 5.79 & 2.52 & 2.51 & 2.49 & 2.43 & 2.43 \\
\hline $\mathrm{L}$ rostral anterior cingulate & -74.9 & 6.10 & 5.86 & 5.82 & 5.76 & 5.71 & 2.11 & 2.11 & 2.16 & 2.11 & 2.10 \\
\hline $\mathrm{R}$ rostral anterior cingulate & -77.4 & 6.20 & 5.96 & 5.90 & 5.86 & 5.78 & 1.66 & 1.66 & 1.66 & 1.66 & 1.66 \\
\hline$L$ rostral middle frontal & -30.7 & 6.15 & 6.00 & 5.98 & 5.96 & 5.93 & 2.47 & 2.47 & 2.45 & 2.44 & 2.45 \\
\hline $\mathrm{R}$ rostral middle frontal & -33.1 & 6.26 & 6.04 & 5.97 & 5.94 & 5.87 & 1.65 & 1.70 & 1.71 & 1.72 & 1.77 \\
\hline L superior frontal & -30.4 & 6.18 & 6.03 & 5.97 & 5.96 & 5.91 & 2.51 & 2.54 & 2.54 & 2.48 & 2.51 \\
\hline R superior frontal & -34.2 & 6.16 & 6.02 & 5.97 & 5.92 & 5.91 & 1.95 & 2.05 & 2.08 & 1.99 & 2.02 \\
\hline L superior parietal & -35.2 & 6.02 & 5.85 & 5.83 & 5.81 & 5.82 & 2.69 & 2.77 & 2.77 & 2.78 & 2.78 \\
\hline $\mathrm{R}$ superior parietal & -34.2 & 6.05 & 5.84 & 5.81 & 5.78 & 5.74 & 2.21 & 2.01 & 2.01 & 1.99 & 2.04 \\
\hline L superior temporal & -39.9 & 6.13 & 5.93 & 5.86 & 5.85 & 5.83 & 2.71 & 2.76 & 2.76 & 2.80 & 2.82 \\
\hline R superior temporal & -39.9 & 6.05 & 5.84 & 5.80 & 5.78 & 5.71 & 1.68 & 1.61 & 1.68 & 1.69 & 1.69 \\
\hline L supramarginal & -33.6 & 6.03 & 5.81 & 5.80 & 5.79 & 5.74 & 2.70 & 2.72 & 2.76 & 2.81 & 2.79 \\
\hline R supramarginal & -32.5 & 6.01 & 5.82 & 5.76 & 5.71 & 5.66 & 1.97 & 1.99 & 2.03 & 2.04 & 2.02 \\
\hline L temporal pole & -75.9 & 6.23 & 6.01 & 6.02 & 5.96 & 5.93 & 2.63 & 2.68 & 2.71 & 2.72 & 2.75 \\
\hline R temporal pole & -74.3 & 6.16 & 5.96 & 5.91 & 5.91 & 5.80 & 1.92 & 1.96 & 1.98 & 2.01 & 2.03 \\
\hline $\mathrm{L}$ transverse temporal & -65.1 & 6.05 & 5.90 & 5.86 & 5.84 & 5.82 & 2.72 & 2.74 & 2.77 & 2.72 & 2.74 \\
\hline $\mathrm{R}$ transverse temporal & -67.3 & 6.06 & 5.83 & 5.81 & 5.82 & 5.79 & 1.67 & 1.63 & 1.67 & 1.69 & 1.69 \\
\hline
\end{tabular}

Note: This table shows the DPF and PPF for several fNIRS-accessible anatomical regions in the head of male subjects. The sensitivity of the region to fNIRS is provided in decibels $(\mathrm{dB} ; 10 \times \log 10)$ and indicates the fraction of photons normalized to the incidence power reaching this region from the scalp at a source-detector distance of $30 \mathrm{~mm}$. 
Table 11 Nearest 10-5 location for recording Brodmann areas (females).

\begin{tabular}{|c|c|c|c|c|c|c|c|c|}
\hline \multirow[b]{2}{*}{ BA } & \multirow[b]{2}{*}{ Region } & \multirow{2}{*}{$\begin{array}{c}\text { Depth } \\
\text { Med (min to max) }\end{array}$} & \multicolumn{2}{|c|}{ Position 1} & \multicolumn{2}{|c|}{ Position 2} & \multicolumn{2}{|c|}{ Position 3} \\
\hline & & & Name & Depth & Name & Depth & Name & Depth \\
\hline BA-1 & L prim. somatosensory ctx & 17.74 (9.83 to 26.80$)$ & C3h & 18.83 & $\mathrm{C} 3$ & 18.86 & $\mathrm{C} 1$ & 21.91 \\
\hline BA-1 & R prim. somatosensory ctx & 16.66 (10.67 to 21.46$)$ & $\mathrm{C} 4$ & 16.58 & $\mathrm{C} 4 \mathrm{~h}$ & 18.52 & $\mathrm{C} 2$ & 21.39 \\
\hline BA-2 & L prim. somatosensory ctx & 19.51 (13.90 to 26.80 ) & C3 & 18.61 & C3h & 20.97 & $\mathrm{C} 1$ & 22.91 \\
\hline BA-2 & R prim. somatosensory ctx & 18.68 (14.29 to 25.25$)$ & $\mathrm{C} 4$ & 15.81 & $\mathrm{C} 4 \mathrm{~h}$ & 21.27 & $\mathrm{C} 2$ & 22.61 \\
\hline BA-3 & L prim. somatosensory ctx & 21.04 (15.31 to 27.58$)$ & FCC3h & 22.31 & $\mathrm{C} 1$ & 23.35 & C3h & 23.50 \\
\hline BA-3 & R prim. somatosensory ctx & 17.49 (14.38 to 24.37$)$ & $\mathrm{C} 4$ & 18.17 & $\mathrm{C} 2$ & 22.14 & $\mathrm{C} 2 \mathrm{~h}$ & 22.33 \\
\hline BA-4 & L prim. motor ctx & 17.23 (13.92 to 26.61$)$ & FCC5h & 19.14 & $\mathrm{C} 1 \mathrm{~h}$ & 19.40 & FCC3 & 19.84 \\
\hline BA-4 & R prim. motor ctx & $18.42(12.92$ to 25.18$)$ & $\mathrm{C} 2 \mathrm{~h}$ & 20.02 & FCC4h & 23.31 & $\mathrm{C} 4 \mathrm{~h}$ & 23.65 \\
\hline BA-5 & $\mathrm{L}$ somatosensory assoc. ctx & 21.88 (17.56 to 28.54$)$ & C1h & 22.92 & $\mathrm{CCPz}$ & 25.65 & $\mathrm{Cz}$ & 28.59 \\
\hline BA-5 & R somatosensory assoc. ctx & $22.30(17.25$ to 29.13$)$ & CCP2 & 22.58 & CCP2h & 22.69 & $\mathrm{C} 2 \mathrm{~h}$ & 26.14 \\
\hline BA-6 & L motor and suppl. motor ctx & 20.53 (16.11 to 25.98$)$ & FCC1h & 22.26 & FCC1 & 22.58 & $\mathrm{FCCz}$ & 26.42 \\
\hline BA-6 & $\mathrm{R}$ motor and suppl. motor ctx & 18.57 (14.59 to 24.12$)$ & $\mathrm{FCC} 4$ & 17.50 & FCC2h & 21.81 & FCC2 & 22.36 \\
\hline BA-7 & L somatosensory assoc. ctx & 23.36 (19.07 to 29.61$)$ & CP1 & 22.33 & CP1h & 23.50 & CCP1h & 24.14 \\
\hline BA-7 & $\mathrm{R}$ somatosensory assoc. ctx & 23.17 (19.08 to 30.78$)$ & $\mathrm{CP} 2$ & 22.49 & CP2h & 22.60 & $\mathrm{CCP} 2 \mathrm{~h}$ & 24.08 \\
\hline BA-8 & $L$ includes frontal eye fields & 17.65 (11.58 to 24.82 ) & F1h & 18.42 & FFC1h & 19.25 & FFC1 & 19.25 \\
\hline BA-8 & $R$ includes frontal eye fields & 18.73 (12.52 to 23.75$)$ & $\mathrm{FC} 4 \mathrm{~h}$ & 19.60 & FFC2h & 20.49 & FFC2 & 21.10 \\
\hline BA-9 & $\mathrm{L}$ dorsolateral prefrontal ctx & 20.25 (13.85 to 25.88 ) & $\mathrm{F} 1$ & 19.30 & FCC3 & 23.50 & FC3 & 24.01 \\
\hline BA-9 & $\mathrm{R}$ dorsolateral prefrontal ctx & 19.29 (14.34 to 25.82$)$ & $\mathrm{FC} 4$ & 22.04 & $\mathrm{FCC} 4$ & 22.51 & $\mathrm{FC} 4 \mathrm{~h}$ & 22.65 \\
\hline BA-10 & $\mathrm{L}$ frontopolar area & 19.11 (14.97 to 25.12$)$ & F3 & 17.92 & AFF1 & 23.95 & AFF1h & 24.01 \\
\hline $\mathrm{BA}-10$ & $\mathrm{R}$ frontopolar area & $18.19(15.34$ to 23.34$)$ & $\mathrm{F} 4$ & 17.58 & AFF2 & 21.14 & AFF2h & 21.60 \\
\hline BA-11 & $\mathrm{L}$ orbitofrontal area & 33.41 (28.12 to 39.29$)$ & AFp7 & 33.09 & Fp1h & 34.63 & Fp1 & 36.82 \\
\hline BA-11 & $\mathrm{R}$ orbitofrontal area & 30.21 (25.89 to 38.14$)$ & F6 & 25.38 & Fp2 & 35.30 & AFp8 & 37.84 \\
\hline BA-17 & L prim. visual ctx (V1) & $16.78(11.62$ to 26.78$)$ & POO1 & 15.54 & PO1 & 17.62 & P01h & 18.74 \\
\hline BA-17 & R prim. visual ctx (V1) & $18.74(14.12$ to 25.58$)$ & PO2 & 16.87 & $\mathrm{PO} 4 \mathrm{~h}$ & 17.11 & $\mathrm{PO} 2 \mathrm{~h}$ & 18.08 \\
\hline $\mathrm{BA}-18$ & L visual assoc. ctx (V2) & 18.44 (15.39 to 24.39$)$ & РPO3h & 16.87 & PPO1h & 18.77 & P01h & 20.92 \\
\hline BA-18 & $\mathrm{R}$ visual assoc. ctx (V2) & 18.54 (15.78 to 25.04$)$ & PPO4h & 16.71 & PPO2 & 17.32 & PPO2h & 20.19 \\
\hline BA-19 & $\mathrm{L}$ associative visual ctx (V3) & 19.96 (16.78 to 24.85$)$ & P1 & 20.43 & CPP3 & 20.73 & CPP5h & 21.83 \\
\hline BA-19 & $\mathrm{R}$ associative visual ctx (V3) & $18.92(16.35$ to 24.77$)$ & P4h & 18.80 & $\mathrm{P} 2$ & 20.44 & $\mathrm{P} 2 \mathrm{~h}$ & 22.73 \\
\hline BA-20 & $\mathrm{L}$ inferior temporal gyrus & 25.54 (21.70 to 34.18$)$ & CCP5 & 27.47 & T7h & 31.55 & C5 & 34.81 \\
\hline BA-20 & $\mathrm{R}$ inferior temporal gyrus & 25.99 (19.92 to 33.58$)$ & CCP6 & 24.14 & C6 & 27.86 & T8h & 30.48 \\
\hline BA-21 & $\mathrm{L}$ middle temporal gyrus & 21.60 (17.42 to 28.05$)$ & CP5h & 21.87 & CCP5 & 22.68 & C5 & 24.33 \\
\hline BA-21 & $\mathrm{R}$ middle temporal gyrus & 18.62 (13.02 to 25.89$)$ & CCP6 & 17.52 & C6 & 20.17 & FCC6 & 23.26 \\
\hline BA-22 & L superior temporal gyrus & 24.08 (20.95 to 27.83 ) & CCP5h & 23.16 & $\mathrm{C} 5 \mathrm{~h}$ & 27.15 & FCC5h & 30.81 \\
\hline
\end{tabular}


Table 11 (Continued).

\begin{tabular}{|c|c|c|c|c|c|c|c|c|}
\hline \multirow[b]{2}{*}{ BA } & \multirow[b]{2}{*}{ Region } & \multirow{2}{*}{$\frac{\text { Depth }}{\text { Med (min to } \max )}$} & \multicolumn{2}{|c|}{ Position 1} & \multicolumn{2}{|c|}{ Position 2} & \multicolumn{2}{|c|}{ Position 3} \\
\hline & & & Name & Depth & Name & Depth & Name & Depth \\
\hline BA-22 & R superior temporal gyrus & $23.02(17.13$ to 29.15$)$ & CCP6h & 21.63 & FCC6h & 26.27 & C6h & 27.08 \\
\hline BA-23 & $\mathrm{L}$ ventral post. cingulate $\mathrm{ctx}$ & $56.82(46.48$ to 63.40$)$ & $\mathrm{FCCz}$ & 46.02 & $\mathrm{Cz}$ & 46.94 & FCC1h & 47.40 \\
\hline BA-23 & $\mathrm{R}$ ventral post. cingulate $\mathrm{ctx}$ & 44.10 (35.34 to 51.29$)$ & FCC2h & 42.34 & $\mathrm{C} 2 \mathrm{~h}$ & 42.53 & $\mathrm{C} 2$ & 43.82 \\
\hline BA-24 & $\mathrm{L}$ ventral ant. cingulate ctx & 47.76 (35.43 to 56.48$)$ & FCC1h & 40.09 & FC1h & 40.57 & $\mathrm{FCz}$ & 43.47 \\
\hline BA-24 & $\mathrm{R}$ ventral ant. cingulate ctx & 38.18 (28.41 to 45.94$)$ & C6h & 34.65 & FC2h & 38.13 & FCC2h & 38.81 \\
\hline BA-25 & L subgenual ctx & 57.24 (46.45 to 61.82$)$ & $\mathrm{F} 1$ & 43.01 & F1h & 43.26 & AFF1h & 44.42 \\
\hline BA-25 & $\mathrm{R}$ subgenual $\mathrm{ct} x$ & 53.07 (27.08 to 61.68$)$ & FFC4 & 33.41 & FC6h & 33.94 & FC4 & 35.13 \\
\hline BA-28 & L post. entorhinal ctx & 44.83 (38.39 to 53.50$)$ & FCC5 & 44.92 & FTT7h & 45.19 & T7h & 46.43 \\
\hline BA-28 & $R$ post. entorhinal ctx & 42.91 (35.51 to 49.62$)$ & FCC6 & 42.79 & T8h & 44.40 & FTT8h & 44.97 \\
\hline BA-29 & $\mathrm{L}$ retrospleniendral cingular ctx & 48.81 (39.72 to 55.55$)$ & CPP1h & 42.29 & $\mathrm{Pz}$ & 42.75 & CPPz & 43.08 \\
\hline BA-29 & $\mathrm{R}$ retrosplenial cingular ctx & 47.77 (40.83 to 53.92$)$ & $\mathrm{CPz}$ & 43.79 & CPP2h & 44.13 & CP2h & 44.69 \\
\hline BA-30 & $L$ part of cingular ctx & $45.68(40.97$ to 51.42$)$ & PPOz & 39.63 & P3h & 41.55 & P3 & 42.41 \\
\hline BA-30 & $\mathrm{R}$ part of cingular ctx & $44.24(40.28$ to 50.43$)$ & P4h & 40.28 & P4 & 42.00 & CPP6h & 43.56 \\
\hline BA-33 & $L$ part of ant. cingulate gyrus & 44.31 (33.02 to 53.70$)$ & FC1h & 38.46 & FFC1 & 40.50 & FFC1h & 41.16 \\
\hline BA-33 & $\mathrm{R}$ part of ant. cingulate gyrus & 36.60 (22.79 to 41.50$)$ & FC4 & 33.49 & FC2h & 38.00 & FFC2h & 38.33 \\
\hline BA-34 & $\mathrm{L}$ ant. entorhinal ctx & 59.50 (51.99 to 64.17$)$ & T7h & 47.81 & FTT7h & 47.88 & FCC5 & 47.98 \\
\hline BA-34 & $\mathrm{R}$ ant. entorhinal ctx & 52.41 (45.77 to 58.64$)$ & C6 & 45.97 & FCC6 & 45.98 & FTT8h & 46.85 \\
\hline BA-35 & $\mathrm{L}$ perirhinal ctx & 50.42 (46.74 to 56.03 ) & CP5 & 47.13 & CCP5 & 47.71 & C5 & 48.48 \\
\hline BA-35 & $\mathrm{R}$ perirhinal ctx & 45.22 (38.15 to 54.14$)$ & CCP6 & 44.12 & CP6 & 44.86 & C6 & 45.09 \\
\hline BA-36 & L parahippocampal ctx & 41.38 (38.26 to 46.72$)$ & TP7h & 39.01 & CP5 & 41.41 & CCP5 & 43.67 \\
\hline BA-36 & $\mathrm{R}$ parahippocampal ctx & 38.09 (31.44 to 44.12$)$ & CP6 & 32.80 & CCP6 & 39.48 & C6 & 40.59 \\
\hline BA-37 & L fusiform gyrus & $17.22(11.79$ to 24.04$)$ & CPP5h & 17.98 & CPP5 & 18.82 & CP5 & 19.83 \\
\hline BA-37 & R fusiform gyrus & $16.62(11.57$ to 23.33$)$ & CP6 & 16.59 & CP6h & 18.37 & CPP6h & 19.85 \\
\hline BA-38 & $\mathrm{L}$ temporopolar area & 32.44 (27.60 to 41.44$)$ & FC5 & 31.97 & FT7h & 34.97 & FTT7h & 37.19 \\
\hline BA-38 & $\mathrm{R}$ temporopolar area & $29.50(22.72$ to 36.56$)$ & FC6 & 29.13 & FCC6 & 29.72 & FTT8h & 34.47 \\
\hline BA-39 & $\mathrm{L}$ angular gyrus & 18.91 (14.61 to 26.24$)$ & CP3 & 19.53 & CPP3 & 19.58 & CP3h & 21.81 \\
\hline BA-39 & $\mathrm{R}$ angular gyrus & 18.40 (12.94 to 25.00$)$ & CP4h & 18.94 & $\mathrm{CP} 4$ & 19.63 & CPP4 & 19.91 \\
\hline BA-40 & L supramarginal gyrus & 20.14 (16.16 to 25.98$)$ & CCP3 & 19.49 & CCP3h & 22.03 & C3h & 23.20 \\
\hline BA-40 & $\mathrm{R}$ supramarginal gyrus & 20.10 (15.64 to 26.12$)$ & CCP4 & 18.71 & CCP4h & 22.57 & C4h & 22.84 \\
\hline BA-42 & L prim. and auditory assoc. ctx & 24.85 (16.20 to 29.57$)$ & C5h & 24.54 & CCP5h & 25.97 & FCC5h & 28.06 \\
\hline BA-42 & R prim. and auditory assoc. ctx & 20.78 (12.53 to 27.26$)$ & C6h & 21.51 & CCP6h & 22.05 & $\mathrm{C} 4$ & 23.10 \\
\hline BA-43 & L subcentral area & 14.98 (8.86 to 24.00$)$ & $\mathrm{C} 5 \mathrm{~h}$ & 15.33 & FCC5h & 16.68 & FCC3 & 21.84 \\
\hline BA-43 & $\mathrm{R}$ subcentral area & 12.37 (5.01 to 20.88 ) & C6h & 15.14 & C4 & 17.10 & FCC4 & 19.85 \\
\hline
\end{tabular}


Table 11 (Continued).

\begin{tabular}{|c|c|c|c|c|c|c|c|c|}
\hline \multirow[b]{2}{*}{ BA } & \multirow[b]{2}{*}{ Region } & \multirow{2}{*}{$\frac{\text { Depth }}{\text { Med (min to } \max )}$} & \multicolumn{2}{|c|}{ Position 1} & \multicolumn{2}{|c|}{ Position 2} & \multicolumn{2}{|c|}{ Position 3} \\
\hline & & & Name & Depth & Name & Depth & Name & Depth \\
\hline BA-44 & L pars opercularis & 18.53 (13.47 to 25.58$)$ & FC5h & 19.22 & FCC5h & 19.58 & FC3 & 22.17 \\
\hline BA-44 & R pars opercularis & 17.85 (8.33 to 25.40$)$ & FCC6h & 18.83 & FC6h & 18.89 & FC4 & 21.75 \\
\hline BA-45 & $\mathrm{L}$ pars triangularis & 16.27 (12.91 to 24.41$)$ & FC5h & 16.64 & FFC5h & 18.03 & FC3 & 20.59 \\
\hline BA-45 & $\mathrm{R}$ pars triangularis & 14.26 (6.86 to 22.95$)$ & FC6h & 15.77 & FFC6h & 16.57 & FC4 & 17.23 \\
\hline BA-46 & $\mathrm{L}$ dorsolateral prefrontal ctx & 17.54 (13.29 to 24.16$)$ & FFC3 & 17.96 & FFC5h & 18.82 & FC3 & 19.46 \\
\hline BA-46 & $\mathrm{R}$ dorsolateral prefrontal ctx & 16.75 (12.02 to 23.75$)$ & FFC6h & 17.15 & FFC4 & 18.62 & FC4 & 19.60 \\
\hline BA-47 & $L$ inferior prefrontal gyrus & 30.20 (25.36 to 37.90$)$ & FFC5 & 24.76 & FC5 & 26.12 & FFC5h & 29.52 \\
\hline BA-47 & $\mathrm{R}$ inferior prefrontal gyrus & 29.08 (19.95 to 35.88$)$ & FFC6h & 26.87 & FC6h & 27.59 & FC6 & 31.25 \\
\hline
\end{tabular}

Note: This table provides the depth (median and range) of several Brodmann areas in female subjects. For each region, the nearest three 10-5 coordinate positions and the depth of the region to this position is provided as guidance for the placement of fNIRS sensors.

Table 12 Nearest 10-5 location for recording Brodmann areas (males).

\begin{tabular}{|c|c|c|c|c|c|c|c|c|}
\hline \multirow[b]{2}{*}{ BA } & \multirow[b]{2}{*}{ Region } & \multirow{2}{*}{$\frac{\text { Depth }}{\text { Med (min to } \max )}$} & \multicolumn{2}{|c|}{ Position 1} & \multicolumn{2}{|c|}{ Position 2} & \multicolumn{2}{|c|}{ Position 3} \\
\hline & & & Name & Depth & Name & Depth & Name & Depth \\
\hline BA-1 & L prim. somatosensory ctx & 17.74 (9.83 to 26.80$)$ & C3h & 18.83 & $\mathrm{C} 3$ & 18.86 & C1 & 21.91 \\
\hline BA-1 & R prim. somatosensory ctx & $16.66(10.67$ to 21.46$)$ & $\mathrm{C} 4$ & 16.58 & $\mathrm{C} 4 \mathrm{~h}$ & 18.52 & $\mathrm{C} 2$ & 21.39 \\
\hline BA-2 & L prim. somatosensory ctx & 19.51 (13.90 to 26.80$)$ & $\mathrm{C} 3$ & 18.61 & C3h & 20.97 & C1 & 22.91 \\
\hline BA-2 & R prim. somatosensory ctx & 18.68 (14.29 to 25.25$)$ & $\mathrm{C} 4$ & 15.81 & $\mathrm{C} 4 \mathrm{~h}$ & 21.27 & $\mathrm{C} 2$ & 22.61 \\
\hline BA-3 & L prim. somatosensory ctx & 21.04 (15.31 to 27.58$)$ & FCC3h & 22.31 & $\mathrm{C} 1$ & 23.35 & C3h & 23.50 \\
\hline BA-3 & R prim. somatosensory ctx & 17.49 (14.38 to 24.37$)$ & $\mathrm{C} 4$ & 18.17 & $\mathrm{C} 2$ & 22.14 & $\mathrm{C} 2 \mathrm{~h}$ & 22.33 \\
\hline $\mathrm{BA}-4$ & L prim. motor ctx & 17.23 (13.92 to 26.61$)$ & FCC5h & 19.14 & $\mathrm{C} 1 \mathrm{~h}$ & 19.40 & FCC3 & 19.84 \\
\hline BA-4 & R prim. motor ctx & $18.42(12.92$ to 25.18$)$ & $\mathrm{C} 2 \mathrm{~h}$ & 20.02 & FCC4h & 23.31 & $\mathrm{C} 4 \mathrm{~h}$ & 23.65 \\
\hline BA-5 & L somatosensory assoc. ctx & 21.88 (17.56 to 28.54$)$ & C1h & 22.92 & $\mathrm{CCPz}$ & 25.65 & $\mathrm{Cz}$ & 28.59 \\
\hline BA-5 & $\mathrm{R}$ somatosensory assoc. ctx & 22.30 (17.25 to 29.13$)$ & CCP2 & 22.58 & CCP2h & 22.69 & $\mathrm{C} 2 \mathrm{~h}$ & 26.14 \\
\hline BA-6 & L motor and suppl. motor ctx & 20.53 (16.11 to 25.98$)$ & FCC1h & 22.26 & FCC1 & 22.58 & $\mathrm{FCCz}$ & 26.42 \\
\hline BA-6 & $\mathrm{R}$ motor and suppl. motor ctx & 18.57 (14.59 to 24.12$)$ & $\mathrm{FCC} 4$ & 17.50 & FCC2h & 21.81 & FCC2 & 22.36 \\
\hline BA-7 & L somatosensory assoc. ctx & 23.36 (19.07 to 29.61$)$ & $\mathrm{CP} 1$ & 22.33 & CP1h & 23.50 & CCP1h & 24.14 \\
\hline BA-7 & $\mathrm{R}$ somatosensory assoc. ctx & 23.17 (19.08 to 30.78$)$ & CP2 & 22.49 & CP2h & 22.60 & CCP2h & 24.08 \\
\hline BA-8 & $L$ includes frontal eye fields & 17.65 (11.58 to 24.82$)$ & $\mathrm{F} 1 \mathrm{~h}$ & 18.42 & FFC1h & 19.25 & FFC1 & 19.25 \\
\hline BA-8 & $R$ includes frontal eye fields & 18.73 (12.52 to 23.75$)$ & FC4h & 19.60 & FFC2h & 20.49 & FFC2 & 21.10 \\
\hline BA-9 & $\mathrm{L}$ dorsolateral prefrontal ctx & 20.25 (13.85 to 25.88$)$ & $\mathrm{F} 1$ & 19.30 & FCC3 & 23.50 & FC3 & 24.01 \\
\hline BA-9 & $\mathrm{R}$ dorsolateral prefrontal ctx & 19.29 (14.34 to 25.82$)$ & FC4 & 22.04 & FCC4 & 22.51 & $\mathrm{FC} 4 \mathrm{~h}$ & 22.65 \\
\hline BA-10 & $\mathrm{L}$ frontopolar area & 19.11 (14.97 to 25.12$)$ & F3 & 17.92 & AFF1 & 23.95 & AFF1h & 24.01 \\
\hline
\end{tabular}


Table 12 (Continued).

\begin{tabular}{|c|c|c|c|c|c|c|c|c|}
\hline \multirow[b]{2}{*}{ BA } & \multirow[b]{2}{*}{ Region } & \multirow{2}{*}{$\frac{\text { Depth }}{\text { Med (min to max })}$} & \multicolumn{2}{|c|}{ Position 1} & \multicolumn{2}{|c|}{ Position 2} & \multicolumn{2}{|c|}{ Position 3} \\
\hline & & & Name & Depth & Name & Depth & Name & Depth \\
\hline BA-10 & $\mathrm{R}$ frontopolar area & 18.19 (15.34 to 23.34$)$ & $\mathrm{F} 4$ & 17.58 & AFF2 & 21.14 & AFF2h & 21.60 \\
\hline $\mathrm{BA}-11$ & $\mathrm{~L}$ orbitofrontal area & 33.41 (28.12 to 39.29$)$ & AFp7 & 33.09 & Fp1h & 34.63 & Fp1 & 36.82 \\
\hline BA-11 & $\mathrm{R}$ orbitofrontal area & 30.21 (25.89 to 38.14$)$ & F6 & 25.38 & Fp2 & 35.30 & AFp8 & 37.84 \\
\hline BA-17 & L prim. visual ctx (V1) & $16.78(11.62$ to 26.78$)$ & POO1 & 15.54 & PO1 & 17.62 & P01h & 18.74 \\
\hline BA-17 & R prim. visual ctx (V1) & $18.74(14.12$ to 25.58$)$ & PO2 & 16.87 & PO4h & 17.11 & $\mathrm{PO} 2 \mathrm{~h}$ & 18.08 \\
\hline BA-18 & L visual assoc. ctx (V2) & 18.44 (15.39 to 24.39 ) & PPO3h & 16.87 & PPO1h & 18.77 & P01h & 20.92 \\
\hline BA-18 & $\mathrm{R}$ visual assoc. ctx (V2) & $18.54(15.78$ to 25.04$)$ & PPO4h & 16.71 & PPO2 & 17.32 & PPO2h & 20.19 \\
\hline BA-19 & $\mathrm{L}$ associative visual ctx (V3) & 19.96 (16.78 to 24.85$)$ & $\mathrm{P} 1$ & 20.43 & CPP3 & 20.73 & CPP5h & 21.83 \\
\hline BA-19 & $\mathrm{R}$ associative visual ctx (V3) & $18.92(16.35$ to 24.77$)$ & P4h & 18.80 & P2 & 20.44 & $\mathrm{P} 2 \mathrm{~h}$ & 22.73 \\
\hline BA-20 & $\mathrm{L}$ inferior temporal gyrus & 25.54 (21.70 to 34.18$)$ & CCP5 & 27.47 & T7h & 31.55 & C5 & 34.81 \\
\hline BA-20 & $\mathrm{R}$ inferior temporal gyrus & 25.99 (19.92 to 33.58$)$ & CCP6 & 24.14 & C6 & 27.86 & T8h & 30.48 \\
\hline BA-21 & $\mathrm{L}$ middle temporal gyrus & 21.60 (17.42 to 28.05$)$ & CP5h & 21.87 & CCP5 & 22.68 & C5 & 24.33 \\
\hline BA-21 & $\mathrm{R}$ middle temporal gyrus & $18.62(13.02$ to 25.89$)$ & CCP6 & 17.52 & C6 & 20.17 & FCC6 & 23.26 \\
\hline BA-22 & L superior temporal gyrus & 24.08 (20.95 to 27.83$)$ & CCP5h & 23.16 & C5h & 27.15 & FCC5h & 30.81 \\
\hline BA-22 & $\mathrm{R}$ superior temporal gyrus & $23.02(17.13$ to 29.15$)$ & CCP6h & 21.63 & FCC6h & 26.27 & C6h & 27.08 \\
\hline BA-23 & $\mathrm{L}$ ventral post. cingulate ctx & $56.82(46.48$ to 63.40$)$ & $\mathrm{FCCz}$ & 46.02 & $\mathrm{Cz}$ & 46.94 & FCC1h & 47.40 \\
\hline BA-23 & $\mathrm{R}$ ventral post. cingulate ctx & 44.10 (35.34 to 51.29$)$ & FCC2h & 42.34 & $\mathrm{C} 2 \mathrm{~h}$ & 42.53 & $\mathrm{C} 2$ & 43.82 \\
\hline BA-24 & $\mathrm{L}$ ventral ant. cingulate ctx & 47.76 (35.43 to 56.48$)$ & FCC1h & 40.09 & FC1h & 40.57 & $\mathrm{FCz}$ & 43.47 \\
\hline BA-24 & $\mathrm{R}$ ventral ant. cingulate ctx & 38.18 (28.41 to 45.94$)$ & C6h & 34.65 & FC2h & 38.13 & FCC2h & 38.81 \\
\hline BA-25 & L subgenual ctx & 57.24 (46.45 to 61.82$)$ & $\mathrm{F} 1$ & 43.01 & F1h & 43.26 & AFF1h & 44.42 \\
\hline BA-25 & $\mathrm{R}$ subgenual ctx & 53.07 (27.08 to 61.68$)$ & FFC4 & 33.41 & FC6h & 33.94 & FC4 & 35.13 \\
\hline BA-28 & $L$ post. entorhinal ctx & 44.83 (38.39 to 53.50$)$ & FCC5 & 44.92 & FTT7h & 45.19 & T7h & 46.43 \\
\hline BA-28 & $\mathrm{R}$ post. entorhinal ctx & 42.91 (35.51 to 49.62$)$ & FCC6 & 42.79 & T8h & 44.40 & FTT8h & 44.97 \\
\hline BA-29 & $\mathrm{L}$ retrospleniendral cingular ctx & 48.81 (39.72 to 55.55$)$ & CPP1h & 42.29 & $\mathrm{Pz}$ & 42.75 & $\mathrm{CPPz}$ & 43.08 \\
\hline BA-29 & $\mathrm{R}$ retrosplenial cingular ctx & 47.77 (40.83 to 53.92$)$ & $\mathrm{CPz}$ & 43.79 & CPP2h & 44.13 & CP2h & 44.69 \\
\hline BA-30 & $L$ part of cingular ctx & 45.68 (40.97 to 51.42 ) & $\mathrm{PPOz}$ & 39.63 & P3h & 41.55 & P3 & 42.41 \\
\hline BA-30 & $\mathrm{R}$ part of cingular ctx & $44.24(40.28$ to 50.43$)$ & P4h & 40.28 & P4 & 42.00 & CPP6h & 43.56 \\
\hline BA-33 & $\mathrm{L}$ part of ant. cingulate gyrus & 44.31 (33.02 to 53.70$)$ & FC1h & 38.46 & FFC1 & 40.50 & FFC1h & 41.16 \\
\hline BA-33 & $\mathrm{R}$ part of ant. cingulate gyrus & 36.60 (22.79 to 41.50$)$ & FC4 & 33.49 & FC2h & 38.00 & FFC2h & 38.33 \\
\hline BA-34 & $\mathrm{L}$ ant. entorhinal ctx & 59.50 (51.99 to 64.17$)$ & T7h & 47.81 & FTT7h & 47.88 & FCC5 & 47.98 \\
\hline BA-34 & $\mathrm{R}$ ant. entorhinal ctx & 52.41 (45.77 to 58.64$)$ & C6 & 45.97 & FCC6 & 45.98 & FTT8h & 46.85 \\
\hline BA-35 & $\mathrm{L}$ perirhinal ctx & 50.42 (46.74 to 56.03 ) & CP5 & 47.13 & CCP5 & 47.71 & C5 & 48.48 \\
\hline BA-35 & $\mathrm{R}$ perirhinal ctx & $45.22(38.15$ to 54.14$)$ & CCP6 & 44.12 & CP6 & 44.86 & C6 & 45.09 \\
\hline
\end{tabular}


Table 12 (Continued).

\begin{tabular}{|c|c|c|c|c|c|c|c|c|}
\hline \multirow[b]{2}{*}{ BA } & \multirow[b]{2}{*}{ Region } & \multirow{2}{*}{$\frac{\text { Depth }}{\text { Med (min to } \max )}$} & \multicolumn{2}{|c|}{ Position 1} & \multicolumn{2}{|c|}{ Position 2} & \multicolumn{2}{|c|}{ Position 3} \\
\hline & & & Name & Depth & Name & Depth & Name & Depth \\
\hline BA-36 & L parahippocampal ctx & 41.38 (38.26 to 46.72$)$ & TP7h & 39.01 & CP5 & 41.41 & CCP5 & 43.67 \\
\hline BA-36 & R parahippocampal ctx & 38.09 (31.44 to 44.12$)$ & CP6 & 32.80 & CCP6 & 39.48 & C6 & 40.59 \\
\hline BA-37 & $L$ fusiform gyrus & $17.22(11.79$ to 24.04$)$ & CPP5h & 17.98 & CPP5 & 18.82 & CP5 & 19.83 \\
\hline BA-37 & $\mathrm{R}$ fusiform gyrus & $16.62(11.57$ to 23.33$)$ & CP6 & 16.59 & CP6h & 18.37 & CPP6h & 19.85 \\
\hline BA-38 & $\mathrm{L}$ temporopolar area & 32.44 (27.60 to 41.44$)$ & FC5 & 31.97 & FT7h & 34.97 & FTT7h & 37.19 \\
\hline BA-38 & $\mathrm{R}$ temporopolar area & 29.50 (22.72 to 36.56$)$ & FC6 & 29.13 & FCC6 & 29.72 & FTT8h & 34.47 \\
\hline BA-39 & $\mathrm{L}$ angular gyrus & 18.91 (14.61 to 26.24$)$ & CP3 & 19.53 & СРP3 & 19.58 & CP3h & 21.81 \\
\hline BA-39 & $\mathrm{R}$ angular gyrus & 18.40 (12.94 to 25.00$)$ & CP4h & 18.94 & $\mathrm{CP} 4$ & 19.63 & CPP4 & 19.91 \\
\hline $\mathrm{BA}-40$ & L supramarginal gyrus & $20.14(16.16$ to 25.98$)$ & CCP3 & 19.49 & СCP3h & 22.03 & C3h & 23.20 \\
\hline $\mathrm{BA}-40$ & $\mathrm{R}$ supramarginal gyrus & 20.10 (15.64 to 26.12$)$ & CCP4 & 18.71 & CCP4h & 22.57 & C4h & 22.84 \\
\hline BA-42 & L prim. and auditory assoc. ctx & 24.85 (16.20 to 29.57$)$ & C5h & 24.54 & CCP5h & 25.97 & FCC5h & 28.06 \\
\hline BA-42 & R prim. and auditory assoc. ctx & 20.78 (12.53 to 27.26$)$ & C6h & 21.51 & CCP6h & 22.05 & $\mathrm{C} 4$ & 23.10 \\
\hline $\mathrm{BA}-43$ & $\mathrm{~L}$ subcentral area & 14.98 (8.86 to 24.00$)$ & $\mathrm{C} 5 \mathrm{~h}$ & 15.33 & FCC5h & 16.68 & $\mathrm{FCC} 3$ & 21.84 \\
\hline $\mathrm{BA}-43$ & $\mathrm{R}$ subcentral area & 12.37 (5.01 to 20.88$)$ & C6h & 15.14 & $\mathrm{C} 4$ & 17.10 & $\mathrm{FCC} 4$ & 19.85 \\
\hline BA-44 & $\mathrm{L}$ pars opercularis & $18.53(13.47$ to 25.58$)$ & FC5h & 19.22 & FCC5h & 19.58 & FC3 & 22.17 \\
\hline BA-44 & $\mathrm{R}$ pars opercularis & 17.85 (8.33 to 25.40$)$ & FCC6h & 18.83 & FC6h & 18.89 & FC4 & 21.75 \\
\hline BA-45 & $\mathrm{L}$ pars triangularis & 16.27 (12.91 to 24.41$)$ & FC5h & 16.64 & FFC5h & 18.03 & FC3 & 20.59 \\
\hline BA-45 & $\mathrm{R}$ pars triangularis & 14.26 (6.86 to 22.95$)$ & FC6h & 15.77 & FFC6h & 16.57 & FC4 & 17.23 \\
\hline BA-46 & $\mathrm{L}$ dorsolateral prefrontal $\mathrm{ctx}$ & 17.54 (13.29 to 24.16$)$ & FFC3 & 17.96 & FFC5h & 18.82 & FC3 & 19.46 \\
\hline $\mathrm{BA}-46$ & $\mathrm{R}$ dorsolateral prefrontal ctx & 16.75 (12.02 to 23.75$)$ & FFC6h & 17.15 & FFC4 & 18.62 & FC4 & 19.60 \\
\hline $\mathrm{BA}-47$ & $L$ inferior prefrontal gyrus & 30.20 (25.36 to 37.90$)$ & FFC5 & 24.76 & FC5 & 26.12 & FFC5h & 29.52 \\
\hline $\mathrm{BA}-47$ & $\mathrm{R}$ inferior prefrontal gyrus & 29.08 (19.95 to 35.88$)$ & FFC6h & 26.87 & FC6h & 27.59 & FC6 & 31.25 \\
\hline
\end{tabular}

Note: This table provides the depth (median and range) of several Brodmann areas in male subjects. For each region, the nearest three 10-5 coordinate positions and the depth of the region to this position is provided as guidance for the placement of fNIRS sensors.

Table 13 Nearest 10-5 location for recording anatomical areas (females).

\begin{tabular}{|c|c|c|c|c|c|c|c|}
\hline \multirow[b]{2}{*}{ Region } & \multirow{2}{*}{$\frac{\text { Depth }}{\text { Med (min to } \max )}$} & \multicolumn{2}{|c|}{ Position 1} & \multicolumn{2}{|c|}{ Position 2} & \multicolumn{2}{|c|}{ Position 3} \\
\hline & & Name & Depth & Name & Depth & Name & Deptr \\
\hline$L$ banks of the superior temporal sulcus & 23.21 (16.11 to 29.05$)$ & CP5h & 24.15 & CP5 & 24.65 & CCP5 & 25.29 \\
\hline $\mathrm{R}$ banks of the superior temporal sulcus & 23.56 (16.16 to 30.42$)$ & CCP6h & 23.27 & CCP6 & 23.28 & CP6h & 24.67 \\
\hline $\mathrm{L}$ caudal anterior cingulate & 39.28 (33.20 to 47.03$)$ & FC1h & 39.75 & FFC1h & 40.09 & $\mathrm{FFC} 1$ & 40.42 \\
\hline $\mathrm{R}$ caudal anterior cingulate & 39.95 (34.10 to 46.30$)$ & FFC2h & 39.84 & $\mathrm{FC} 2 \mathrm{~h}$ & 40.56 & $\mathrm{FCz}$ & 41.90 \\
\hline L caudal middle frontal & 20.16 (14.04 to 26.27 ) & FC3h & 19.78 & FCC3h & 21.65 & FC3 & 25.11 \\
\hline $\mathrm{R}$ caudal middle frontal & $19.82(13.18$ to 25.70$)$ & $\mathrm{FC2}$ & 20.11 & FCC2 & 21.38 & FC4h & 22.56 \\
\hline
\end{tabular}


Table 13 (Continued).

\begin{tabular}{|c|c|c|c|c|c|c|c|}
\hline \multirow[b]{2}{*}{ Region } & \multirow{2}{*}{$\frac{\text { Depth }}{\operatorname{Med}(\min \text { to } \max )}$} & \multicolumn{2}{|c|}{ Position 1} & \multicolumn{2}{|c|}{ Position 2} & \multicolumn{2}{|c|}{ Position 3} \\
\hline & & Name & Depth & Name & Depth & Name & Depth \\
\hline$L$ cuneus & 28.23 (22.19 to 34.96$)$ & PPO1h & 26.70 & $\mathrm{PPOz}$ & 26.84 & P1h & 29.63 \\
\hline$R$ cuneus & 27.75 (14.83 to 34.30$)$ & PPO2h & 24.58 & $\mathrm{PPOz}$ & 27.04 & $\mathrm{Pz}$ & 28.87 \\
\hline L entorhinal & 41.23 (33.56 to 44.60$)$ & T7h & 44.20 & $\mathrm{T7}$ & 44.97 & FTT7h & 45.15 \\
\hline$R$ entorhinal & 40.97 (32.97 to 45.95$)$ & T8h & 44.42 & FTT8h & 45.18 & T8 & 46.69 \\
\hline$L$ frontal pole & $14.67(10.80$ to 19.21$)$ & AFp1 & 14.31 & AFp3h & 15.06 & Fp1h & 16.14 \\
\hline $\mathrm{R}$ frontal pole & 14.63 (12.08 to 20.75$)$ & AFp4h & 15.14 & AFp2h & 15.57 & Fp2h & 16.44 \\
\hline$L$ fusiform & 31.96 (28.22 to 39.27$)$ & P5 & 29.54 & CPP5 & 33.07 & T7h & 35.53 \\
\hline R fusiform & 32.62 (27.68 to 40.28$)$ & CPP6 & 31.97 & T8h & 35.69 & CP6 & 36.44 \\
\hline $\mathrm{L}$ inferior parietal & 18.27 (14.95 to 21.71$)$ & СРP3h & 18.04 & CP3h & 19.44 & CPP3 & 20.81 \\
\hline $\mathrm{R}$ inferior parietal & 17.98 (14.16 to 23.42$)$ & $\mathrm{CP} 4 \mathrm{~h}$ & 18.51 & $\mathrm{CP} 4$ & 20.01 & CPP4 & 20.12 \\
\hline$L$ inferior temporal & $19.80(15.60$ to 25.35$)$ & CP5 & 18.76 & CPP5 & 19.50 & T7h & 26.28 \\
\hline $\mathrm{R}$ inferior temporal & $18.96(14.27$ to 25.30$)$ & CP6 & 18.36 & CPP6 & 18.78 & T8h & 26.15 \\
\hline$L$ insula & 35.28 (30.83 to 41.73$)$ & FCC5 & 34.27 & FCC5h & 35.36 & C5h & 38.60 \\
\hline$R$ insula & 33.90 (26.95 to 40.54$)$ & FCC6h & 33.80 & FCC6 & 33.84 & C6h & 36.07 \\
\hline $\mathrm{L}$ isthmus cingulate & 53.73 (48.46 to 59.82 ) & CPP1h & 45.46 & CP1h & 46.50 & $\mathrm{CPz}$ & 46.86 \\
\hline $\mathrm{R}$ isthmus cingulate & 53.32 (45.89 to 59.62 ) & $\mathrm{CPPz}$ & 46.69 & $\mathrm{CPz}$ & 46.70 & CPP6h & 47.21 \\
\hline L lateral occipital & $14.29(11.12$ to 21.28$)$ & PPO3h & 15.64 & P5h & 18.67 & CPP5h & 22.28 \\
\hline R lateral occipital & 14.16 (11.54 to 21.02$)$ & PPO4 & 15.58 & PPO4h & 16.04 & P6h & 17.69 \\
\hline L lateral orbitofrontal & 33.21 (28.82 to 42.25$)$ & AFp7 & 34.60 & FFC5 & 34.91 & FC5 & 35.63 \\
\hline $\mathrm{R}$ lateral orbitofrontal & 33.93 (29.23 to 41.80$)$ & FFC6h & 33.96 & FFC6 & 36.12 & FC6 & 37.89 \\
\hline$L$ lingual & 40.05 (33.31 to 47.05$)$ & P001h & 31.42 & $\mathrm{POz}$ & 34.42 & PPO5h & 40.61 \\
\hline $\mathrm{R}$ lingual & 40.64 (35.95 to 46.43$)$ & PPO6h & 39.09 & P6h & 42.05 & CPP6h & 43.89 \\
\hline L medial orbitofrontal & 36.25 (31.39 to 43.95$)$ & Fp1h & 34.31 & Fp1 & 39.01 & AFp7 & 44.25 \\
\hline $\mathrm{R}$ medial orbitofrontal & 37.52 (31.86 to 42.82$)$ & AFp4h & 32.85 & Fp2h & 34.98 & Fp2 & 39.41 \\
\hline $\mathrm{L}$ middle temporal & 15.91 (12.21 to 21.67 ) & CP5 & 15.80 & CCP5 & 20.30 & $\mathrm{C} 5$ & 20.61 \\
\hline $\mathrm{R}$ middle temporal & 15.29 (9.52 to 21.80$)$ & CP6h & 14.71 & CCP6 & 17.19 & C6 & 18.61 \\
\hline L paracentral & 31.27 (26.19 to 37.99 ) & $\mathrm{Cz}$ & 29.01 & CCP1h & 30.74 & $\mathrm{C} 1 \mathrm{~h}$ & 33.38 \\
\hline R paracentral & $31.32(25.47$ to 37.74$)$ & $\mathrm{CCPz}$ & 27.99 & $\mathrm{Cz}$ & 28.07 & $\mathrm{C} 2 \mathrm{~h}$ & 38.65 \\
\hline L parahippocampal & 48.29 (44.53 to 52.38$)$ & TTP7h & 43.48 & CP5 & 44.23 & TP7h & 45.30 \\
\hline R parahippocampal & 47.74 (39.64 to 52.87 ) & T8h & 43.22 & CCP6 & 43.24 & CP6 & 44.06 \\
\hline L pars opercularis & 21.83 (17.86 to 28.37 ) & FC5h & 22.75 & FC3 & 23.87 & FC5 & 24.53 \\
\hline $\mathrm{R}$ pars opercularis & 22.30 (16.78 to 29.90$)$ & FCC6h & 23.33 & FC6h & 23.84 & FC4 & 24.75 \\
\hline L pars orbitalis & 18.73 (13.61 to 29.05$)$ & F5 & 19.09 & FFC5 & 20.11 & FC5 & 20.90 \\
\hline
\end{tabular}


Table 13 (Continued).

\begin{tabular}{|c|c|c|c|c|c|c|c|}
\hline \multirow[b]{2}{*}{ Region } & \multirow{2}{*}{$\frac{\text { Depth }}{\operatorname{Med}(\min \text { to } \max )}$} & \multicolumn{2}{|c|}{ Position 1} & \multicolumn{2}{|c|}{ Position 2} & \multicolumn{2}{|c|}{ Position 3} \\
\hline & & Name & Depth & Name & Depth & Name & Depth \\
\hline $\mathrm{R}$ pars orbitalis & 18.34 (13.32 to 28.56$)$ & F6h & 18.34 & F6 & 19.33 & FFC6 & 19.60 \\
\hline $\mathrm{L}$ pars triangularis & 17.46 (14.39 to 24.87$)$ & FFC5 & 17.98 & FC5h & 19.21 & FFC5h & 20.92 \\
\hline $\mathrm{R}$ pars triangularis & 17.34 (12.67 to 26.09$)$ & FC6h & 18.01 & FFC4 & 19.73 & FFC6h & 20.12 \\
\hline L pericalcarine & 28.08 (23.56 to 38.01$)$ & PO1 & 23.55 & P01h & 25.51 & PPO1h & 31.08 \\
\hline $\mathrm{R}$ pericalcarine & 32.05 (21.59 to 39.28$)$ & $\mathrm{PO} 2$ & 24.32 & PPO2 & 31.93 & PPO2h & 34.21 \\
\hline L postcentral & $18.19(14.12$ to 26.28$)$ & $\mathrm{C} 3$ & 18.81 & C3h & 19.92 & C5h & 20.55 \\
\hline R postcentral & $17.72(13.57$ to 24.70$)$ & $\mathrm{C} 4$ & 18.07 & $\mathrm{C} 4 \mathrm{~h}$ & 19.98 & $\mathrm{C} 2$ & 21.34 \\
\hline L posterior cingulate & $44.62(39.13$ to 50.33$)$ & FCC1h & 42.71 & $\mathrm{C} 1 \mathrm{~h}$ & 43.63 & $\mathrm{C} 1$ & 44.69 \\
\hline $\mathrm{R}$ posterior cingulate & 43.89 (35.48 to 50.37$)$ & FCC2h & 42.31 & $\mathrm{C} 2 \mathrm{~h}$ & 42.74 & $\mathrm{FCCz}$ & 43.52 \\
\hline L precentral & 20.54 (15.71 to 26.01$)$ & FCC5h & 20.53 & FCC3 & 20.84 & $\mathrm{FCC} 3 \mathrm{~h}$ & 22.12 \\
\hline R precentral & $19.80(15.05$ to 26.21$)$ & $\mathrm{FCC} 4$ & 19.33 & FCC4h & 21.07 & FCC2 & 22.86 \\
\hline L precuneus & 35.94 (29.59 to 42.34$)$ & CP1h & 33.50 & CPP1h & 33.92 & $\mathrm{P} 1 \mathrm{~h}$ & 34.74 \\
\hline $\mathrm{R}$ precuneus & $36.48(31.00$ to 41.39$)$ & $\mathrm{CPz}$ & 32.01 & $\mathrm{CPPz}$ & 32.40 & CPP2h & 34.28 \\
\hline $\mathrm{L}$ rostral anterior cingulate & 36.77 (31.08 to 44.50$)$ & AFF1 & 35.23 & AFF1h & 35.68 & AF3 & 37.73 \\
\hline $\mathrm{R}$ rostral anterior cingulate & 40.84 (34.05 to 47.05$)$ & AFF2h & 37.10 & AFF2 & 37.16 & AFFz & 37.63 \\
\hline$L$ rostral middle frontal & 16.65 (12.87 to 21.98$)$ & FFC3 & 18.34 & F3h & 19.18 & FFC3h & 19.42 \\
\hline $\mathrm{R}$ rostral middle frontal & 17.44 (13.68 to 22.37$)$ & FFC4 & 18.91 & $\mathrm{FFC4h}$ & 19.49 & $\mathrm{~F} 4 \mathrm{~h}$ & 19.83 \\
\hline L superior frontal & 20.27 (15.51 to 27.77 ) & FFC1 & 20.91 & FC1h & 22.51 & FCC1h & 24.18 \\
\hline R superior frontal & 21.65 (17.08 to 27.22$)$ & FFC2h & 21.40 & FC2h & 22.66 & $\mathrm{FCC} 2 \mathrm{~h}$ & 23.12 \\
\hline L superior parietal & $21.50(16.07$ to 28.94$)$ & CCP1 & 22.65 & $\mathrm{CP} 1$ & 22.84 & CCP3h & 26.16 \\
\hline $\mathrm{R}$ superior parietal & 21.26 (16.90 to 27.98$)$ & $\mathrm{CP} 2 \mathrm{~h}$ & 20.79 & $\mathrm{CCP} 2$ & 23.82 & $\mathrm{CP} 2$ & 23.85 \\
\hline L superior temporal & 22.33 (18.26 to 27.88 ) & CCP5h & 24.11 & C5 & 24.33 & FCC5 & 24.37 \\
\hline $\mathrm{R}$ superior temporal & $21.82(13.63$ to 28.74$)$ & C6h & 19.10 & FCC6 & 24.31 & C6 & 24.56 \\
\hline L supramarginal & $19.46(16.28$ to 24.64$)$ & CCP3 & 19.85 & C3 & 20.09 & CCP5h & 25.75 \\
\hline R supramarginal & 18.97 (12.41 to 26.09$)$ & C4 & 19.15 & $\mathrm{CCP} 4$ & 19.43 & CCP6h & 25.53 \\
\hline L temporal pole & 35.47 (27.47 to 42.46$)$ & FFT7h & 36.07 & FT7h & 36.68 & FTT7h & 38.98 \\
\hline $\mathrm{R}$ temporal pole & 35.78 (25.70 to 43.27$)$ & FT8h & 36.88 & FC6 & 38.00 & FTT8h & 40.29 \\
\hline $\mathrm{L}$ transverse temporal & 32.22 (28.60 to 36.76$)$ & C5h & 32.05 & C5 & 32.85 & CCP5h & 35.98 \\
\hline $\mathrm{R}$ transverse temporal & 30.89 (23.11 to 39.98$)$ & FCC6h & 26.28 & C6h & 30.59 & CCP6h & 35.07 \\
\hline
\end{tabular}

Note: This table provides the depth (median and range) of several anatomical regions in female subjects. For each region, the nearest three 10-5 coordinate positions and the depth of the region to this position is provided as guidance for the placement of fNIRS sensors. 
Table 14 Nearest 10-5 location for recording anatomical regions (males).

\begin{tabular}{|c|c|c|c|c|c|c|c|}
\hline \multirow[b]{2}{*}{ Region } & \multirow{2}{*}{$\frac{\text { Depth }}{\text { Med (min to } \max )}$} & \multicolumn{2}{|c|}{ Position 1} & \multicolumn{2}{|c|}{ Position 2} & \multicolumn{2}{|c|}{ Position 3} \\
\hline & & Name & Depth & Name & Depth & Name & Depth \\
\hline$L$ banks of the superior temporal sulcus & $21.14(11.90$ to 27.00$)$ & CP5h & 22.06 & CCP5 & 22.16 & CP5 & 23.25 \\
\hline $\mathrm{R}$ banks of the superior temporal sulcus & $24.82(14.22$ to 30.44$)$ & CCP6h & 23.45 & CCP6 & 25.09 & CP6h & 25.12 \\
\hline $\mathrm{L}$ caudal anterior cingulate & 40.99 (28.19 to 46.86$)$ & FC1h & 40.37 & FFC1 & 40.98 & FFC1h & 41.88 \\
\hline $\mathrm{R}$ caudal anterior cingulate & 40.85 (30.59 to 46.40$)$ & FFC2h & 40.65 & $\mathrm{~F} 2 \mathrm{~h}$ & 41.09 & $\mathrm{FC} 2 \mathrm{~h}$ & 41.50 \\
\hline $\mathrm{L}$ caudal middle frontal & 20.58 (11.99 to 26.63$)$ & FC3h & 19.18 & FCC3h & 20.47 & FC3 & 25.09 \\
\hline $\mathrm{R}$ caudal middle frontal & 20.68 (11.36 to 24.36$)$ & FCC2 & 19.75 & $\mathrm{FC} 4 \mathrm{~h}$ & 21.57 & FCC4h & 22.44 \\
\hline $\mathrm{L}$ cuneus & 28.89 (23.94 to 36.05$)$ & $\mathrm{POz}$ & 27.02 & $\mathrm{PPOz}$ & 29.14 & PPO1h & 30.31 \\
\hline$R$ cuneus & 25.89 (17.16 to 34.01$)$ & $\mathrm{POz}$ & 23.80 & $\mathrm{PPOz}$ & 27.69 & PPO2h & 27.89 \\
\hline L entorhinal & 39.86 (30.13 to 43.65$)$ & FTT7h & 44.17 & $\mathrm{~T} 7$ & 44.39 & FTT7 & 45.12 \\
\hline$R$ entorhinal & 39.46 (25.51 to 45.07$)$ & FTT8h & 45.25 & T8 & 45.94 & FTT8 & 45.99 \\
\hline$L$ frontal pole & 14.79 (11.31 to 21.25$)$ & AFp3h & 14.50 & AFp1 & 15.08 & Fp1h & 16.94 \\
\hline $\mathrm{R}$ frontal pole & 15.58 (12.27 to 22.68$)$ & AFp2 & 15.39 & AFp4h & 15.55 & Fp2h & 17.47 \\
\hline$L$ fusiform & 32.59 (28.25 to 36.92$)$ & P5 & 27.23 & CPP5 & 32.60 & TP7h & 37.36 \\
\hline$R$ fusiform & 34.15 (27.93 to 37.82$)$ & P6 & 30.32 & T8h & 36.96 & CP6 & 38.16 \\
\hline $\mathrm{L}$ inferior parietal & 18.17 (13.25 to 22.86$)$ & CPP3h & 17.90 & CPP3 & 19.44 & CPP5h & 23.46 \\
\hline $\mathrm{R}$ inferior parietal & 17.88 (13.18 to 22.46$)$ & CPP4 & 19.32 & CP4 & 20.07 & CPP6h & 22.35 \\
\hline$L$ inferior temporal & 20.37 (16.54 to 28.07 ) & CPP5 & 20.29 & TP7h & 21.79 & TTP7h & 22.41 \\
\hline $\mathrm{R}$ inferior temporal & 20.19 (13.89 to 27.26$)$ & CPP6 & 18.61 & CP6 & 19.28 & T8h & 26.15 \\
\hline$L$ insula & 35.89 (30.59 to 40.19$)$ & FCC5 & 34.43 & FCC5h & 36.74 & $\mathrm{C} 5 \mathrm{~h}$ & 39.99 \\
\hline$R$ insula & 36.18 (29.30 to 40.25$)$ & FCC6 & 34.77 & FC6 & 35.31 & C6h & 39.65 \\
\hline $\mathrm{L}$ isthmus cingulate & 54.38 (47.55 to 59.31$)$ & CPP1h & 46.37 & $\mathrm{P} 1 \mathrm{~h}$ & 46.79 & CP1h & 47.23 \\
\hline $\mathrm{R}$ isthmus cingulate & 54.63 (47.31 to 60.58$)$ & CPP2h & 46.44 & $\mathrm{CPPz}$ & 47.31 & $\mathrm{Pz}$ & 47.63 \\
\hline L lateral occipital & $14.08(11.73$ to 19.74$)$ & PPO5h & 15.27 & P5h & 18.50 & P5 & 18.72 \\
\hline $\mathrm{R}$ lateral occipital & 14.07 (11.69 to 18.85$)$ & PPO4h & 16.01 & PPO4 & 16.63 & P6h & 18.02 \\
\hline$L$ lateral orbitofrontal & 33.40 (30.05 to 37.97$)$ & F5 & 32.91 & FFC5 & 34.98 & AFp7 & 35.56 \\
\hline $\mathrm{R}$ lateral orbitofrontal & 34.17 (29.27 to 39.09$)$ & FFC6h & 34.25 & F6 & 34.41 & FFC6 & 35.79 \\
\hline$L$ lingual & 41.60 (35.07 to 46.59$)$ & P5h & 43.98 & P5 & 44.78 & CPP5 & 45.74 \\
\hline $\mathrm{R}$ lingual & 41.40 (37.28 to 46.82$)$ & $\mathrm{POz}$ & 38.42 & P6h & 43.35 & CPP6h & 43.91 \\
\hline L medial orbitofrontal & 37.24 (30.88 to 43.72$)$ & $\mathrm{Fpz}$ & 33.39 & Fp1h & 36.19 & $\mathrm{Fp} 1$ & 40.68 \\
\hline $\mathrm{R}$ medial orbitofrontal & 38.62 (31.32 to 42.93$)$ & $\mathrm{Fpz}$ & 32.72 & Fp2h & 35.50 & $\mathrm{Fp} 2$ & 39.94 \\
\hline $\mathrm{L}$ middle temporal & $16.32(12.36$ to 20.13$)$ & CP5 & 15.58 & FTT7h & 20.74 & CCP5 & 21.14 \\
\hline $\mathrm{R}$ middle temporal & 17.05 (9.74 to 20.93 ) & CCP6 & 19.13 & C6 & 19.72 & FTT8h & 22.50 \\
\hline L paracentral & 31.12 (20.90 to 35.62$)$ & $\mathrm{Cz}$ & 28.27 & CCP1h & 30.65 & $\mathrm{C} 1 \mathrm{~h}$ & 32.18 \\
\hline $\mathrm{R}$ paracentral & 32.06 (22.04 to 41.07$)$ & $\mathrm{CCPz}$ & 27.17 & $\mathrm{Cz}$ & 28.14 & $\mathrm{C} 2 \mathrm{~h}$ & 38.24 \\
\hline L parahippocampal & 48.31 (43.27 to 53.86$)$ & CP5 & 44.20 & TTP7h & 44.84 & TP7h & 45.61 \\
\hline
\end{tabular}


Table 14 (Continued).

\begin{tabular}{|c|c|c|c|c|c|c|c|}
\hline \multirow[b]{2}{*}{ Region } & \multirow{2}{*}{$\frac{\text { Depth }}{\text { Med }(\min \text { to } \max )}$} & \multicolumn{2}{|c|}{ Position 1} & \multicolumn{2}{|c|}{ Position 2} & \multicolumn{2}{|c|}{ Position 3} \\
\hline & & Name & Depth & Name & Depth & Name & Depth \\
\hline $\mathrm{R}$ parahippocampal & 49.41 (36.90 to 53.63 ) & T8h & 44.15 & CP6 & 44.80 & TTP8h & 45.03 \\
\hline L pars opercularis & 22.15 (15.67 to 27.38$)$ & FC3 & 22.65 & FC5h & 23.34 & FC5 & 24.43 \\
\hline $\mathrm{R}$ pars opercularis & 24.02 (16.57 to 28.39$)$ & FCC4 & 24.70 & FC6h & 24.70 & FC4 & 25.26 \\
\hline L pars orbitalis & 17.71 (13.52 to 23.96$)$ & F5 & 17.57 & FFC5 & 19.26 & F7h & 19.48 \\
\hline $\mathrm{R}$ pars orbitalis & $17.38(13.36$ to 23.16$)$ & F6h & 17.51 & F6 & 18.16 & FFC6 & 18.80 \\
\hline $\mathrm{L}$ pars triangularis & $18.19(13.52$ to 24.03$)$ & FC5h & 17.91 & FFC5 & 19.05 & FFC5h & 21.84 \\
\hline $\mathrm{R}$ pars triangularis & 17.05 (10.76 to 21.18$)$ & FC6h & 17.62 & FFC6 & 18.68 & FFC6h & 20.30 \\
\hline L pericalcarine & 31.03 (24.03 to 37.99$)$ & POO1 & 24.55 & PO1 & 27.94 & P01h & 28.46 \\
\hline $\mathrm{R}$ pericalcarine & 31.31 (23.03 to 38.29$)$ & $\mathrm{PO} 2 \mathrm{~h}$ & 26.53 & $\mathrm{PO} 2$ & 27.66 & PPO2 & 35.70 \\
\hline L postcentral & $18.34(10.85$ to 21.43$)$ & C3 & 18.69 & C3h & 18.83 & $\mathrm{C} 5 \mathrm{~h}$ & 20.26 \\
\hline R postcentral & 18.67 (11.33 to 22.23$)$ & C4 & 17.89 & $\mathrm{C} 4 \mathrm{~h}$ & 19.57 & CCP2 & 21.78 \\
\hline L posterior cingulate & 45.87 (33.51 to 51.01$)$ & FCC1h & 42.41 & $\mathrm{C} 1 \mathrm{~h}$ & 42.59 & $\mathrm{C} 1$ & 44.66 \\
\hline $\mathrm{R}$ posterior cingulate & 45.78 (33.09 to 50.59$)$ & $\mathrm{FCCz}$ & 43.05 & $\mathrm{C} 2 \mathrm{~h}$ & 43.55 & FCC2h & 43.67 \\
\hline L precentral & 20.42 (11.63 to 23.83$)$ & FCC3 & 19.95 & FCC5h & 19.95 & $\mathrm{C} 1$ & 21.99 \\
\hline R precentral & 20.83 (12.58 to 24.50 ) & FCC4 & 19.43 & $\mathrm{C} 2 \mathrm{~h}$ & 21.33 & $\mathrm{C} 2$ & 22.50 \\
\hline$L$ precuneus & 36.38 (31.31 to 41.92$)$ & CP1h & 32.28 & $\mathrm{P} 1 \mathrm{~h}$ & 33.16 & CPP1h & 34.28 \\
\hline $\mathrm{R}$ precuneus & 37.06 (31.98 to 40.83$)$ & $\mathrm{CPPz}$ & 31.99 & $\mathrm{Pz}$ & 33.27 & $\mathrm{P} 2 \mathrm{~h}$ & 34.94 \\
\hline $\mathrm{L}$ rostral anterior cingulate & 39.17 (34.16 to 44.98$)$ & AFF1 & 37.25 & AFF1h & 38.16 & AF3 & 39.13 \\
\hline $\mathrm{R}$ rostral anterior cingulate & 41.57 (34.99 to 48.21$)$ & AFF2h & 38.32 & AFF2 & 38.99 & AFFz & 39.26 \\
\hline$L$ rostral middle frontal & 16.12 (11.35 to 20.22$)$ & F3 & 17.77 & FFC3 & 18.04 & FFC3h & 18.23 \\
\hline $\mathrm{R}$ rostral middle frontal & 17.12 (11.97 to 21.71$)$ & FFC4 & 19.33 & FC4 & 19.48 & FFC4h & 19.65 \\
\hline L superior frontal & 21.12 (10.86 to 24.22$)$ & FFC1h & 19.38 & FC1h & 21.49 & FCC1h & 22.69 \\
\hline R superior frontal & 21.73 (12.48 to 26.70$)$ & FFC2h & 21.41 & $\mathrm{FC} 2 \mathrm{~h}$ & 22.26 & FCC2h & 22.55 \\
\hline L superior parietal & 20.93 (16.82 to 25.99$)$ & $\mathrm{CP} 1$ & 21.13 & CPP1 & 22.33 & CCP1 & 22.53 \\
\hline $\mathrm{R}$ superior parietal & 20.75 (16.19 to 25.34$)$ & CPP2h & 20.54 & CP2h & 21.07 & CP2 & 23.20 \\
\hline L superior temporal & 22.57 (18.86 to 26.22$)$ & FCC5 & 23.19 & C5 & 23.55 & CCP5h & 24.95 \\
\hline R superior temporal & 22.91 (16.21 to 27.73 ) & FCC6 & 23.18 & CCP6h & 23.36 & C6 & 25.65 \\
\hline L supramarginal & $19.61(15.15$ to 24.27$)$ & CCP3 & 19.70 & C5h & 20.75 & CCP5h & 25.09 \\
\hline R supramarginal & 20.28 (13.41 to 24.47$)$ & $\mathrm{C} 4$ & 19.41 & $\mathrm{CCP} 4$ & 19.68 & CCP6h & 27.32 \\
\hline $\mathrm{L}$ temporal pole & 35.35 (25.47 to 41.75$)$ & FT7 & 37.58 & FT7h & 38.97 & FTT7 & 40.60 \\
\hline $\mathrm{R}$ temporal pole & 36.35 (27.70 to 41.17$)$ & FFT8h & 37.00 & FT8h & 39.84 & FTT8h & 41.67 \\
\hline $\mathrm{L}$ transverse temporal & $33.52(23.75$ to 37.96$)$ & C5 & 32.11 & $\mathrm{C} 5 \mathrm{~h}$ & 33.11 & CCP5h & 37.45 \\
\hline $\mathrm{R}$ transverse temporal & $34.22(24.65$ to 38.81$)$ & C6 & 32.98 & C6h & 33.67 & CCP6h & 38.18 \\
\hline
\end{tabular}

Note: This table provides the depth (median and range) of several anatomical regions in male subjects. For each region, the nearest three 10-5 coordinate positions and the depth of the region to this position is provided as guidance for the placement of fNIRS sensors. 
Where Table 3 in the text describes the sensitivity, DPF, and PPF of each wavelength by select anatomical regions, Table 5 describes these values for select Brodmann areas. Similarly, where Table 4 in the text describes the nearest 10-5 locations for select Brodmann areas, Table 6 describes these locations for select anatomical regions.

Tables 3-6 contain our findings for select areas/regions for both sexes and Tables 7-10 are separated by sex.

Tables 7-10 are the complete results of our sensitivity, DPF, and PPF findings specifically divided by sex and explained by either Brodmann areas or anatomical regions. Tables 11-14 are the complete results of the nearest 10-5 locations divided by sex and explained by either Brodmann areas or anatomical regions.

Finally, Table 15 contains the results of our generalized equation for DPF for each 10-5 location.

\section{Appendix A: Generalized Model of the Differential Pathlength Factor}

The earlier works by Scholkmann and Wolf ${ }^{37}$ and Duncan et al. ${ }^{31}$ have suggested equations to estimate the DPF as a function of

Table 15 Generalized equation for DPF for 10-5 locations.

\begin{tabular}{|c|c|c|c|c|c|c|c|c|}
\hline \multirow[b]{2}{*}{ Name } & \multicolumn{8}{|c|}{ Beta values } \\
\hline & $\beta_{0}$ & $\beta_{1}$ & $\beta_{2}$ & $\beta_{3}$ & $\beta_{4}$ & $\beta_{5}$ & $\beta_{6}$ & $R^{2}$ \\
\hline $\mathrm{Fp} 1$ & $7.7 \times 10^{-4}$ & $-9.3 \times 10^{-2}$ & $-3.5 \times 10^{-4}$ & $-1.5 \times 10^{-3}$ & $1.6 \times 10^{-1}$ & $2.7 \times 10^{-7}$ & $-1.6 \times 10^{1}$ & 0.24 \\
\hline $\mathrm{Fpz}$ & $4.9 \times 10^{-03}$ & $-1.9 \times 10^{-1}$ & $8.5 \times 10^{-4}$ & $-1.5 \times 10^{-3}$ & $-3.6 \times 10^{-1}$ & $-6.7 \times 10^{-7}$ & $5.6 \times 10^{1}$ & 0.16 \\
\hline $\mathrm{Fp} 2$ & $1.5 \times 10^{-3}$ & $-8.7 \times 10^{-2}$ & $1.3 \times 10^{-4}$ & $-1.5 \times 10^{-3}$ & $-4.0 \times 10^{-2}$ & $-1.5 \times 10^{-7}$ & $1.2 \times 10^{1}$ & 0.19 \\
\hline AF9 & $4.7 \times 10^{-3}$ & $-4.6 \times 10^{-2}$ & $-2.3 \times 10^{-3}$ & $-2.8 \times 10^{-3}$ & $9.2 \times 10^{-1}$ & $1.9 \times 10^{-6}$ & $-1.2 \times 10^{2}$ & 0.91 \\
\hline AF7 & $2.8 \times 10^{-3}$ & $-3.5 \times 10^{-1}$ & $-4.0 \times 10^{-3}$ & $-2.1 \times 10^{-3}$ & $1.5 \times 10^{0}$ & $3.5 \times 10^{-6}$ & $-1.9 \times 10^{2}$ & 0.24 \\
\hline AF5 & $7.0 \times 10^{-4}$ & $-1.1 \times 10^{-1}$ & $8.3 \times 10^{-5}$ & $-9.8 \times 10^{-4}$ & $-2.0 \times 10^{-2}$ & $-9.4 \times 10^{-8}$ & $7.4 \times 10^{0}$ & 0.17 \\
\hline AF3 & $1.0 \times 10^{-3}$ & $-9.8 \times 10^{-2}$ & $1.9 \times 10^{-4}$ & $-1.1 \times 10^{-3}$ & $-6.0 \times 10^{-2}$ & $-1.8 \times 10^{-7}$ & $1.3 \times 10^{1}$ & 0.18 \\
\hline $\mathrm{AF} 1$ & $1.3 \times 10^{-3}$ & $-1.1 \times 10^{-1}$ & $-2.3 \times 10^{-5}$ & $-1.2 \times 10^{-3}$ & $1.4 \times 10^{-2}$ & $1.2 \times 10^{-8}$ & $3.8 \times 10^{0}$ & 0.17 \\
\hline $\mathrm{AFz}$ & $1.5 \times 10^{-3}$ & $-1.2 \times 10^{-1}$ & $-6.5 \times 10^{-4}$ & $-1.4 \times 10^{-3}$ & $2.6 \times 10^{-1}$ & $5.5 \times 10^{-7}$ & $-2.7 \times 10^{1}$ & 0.19 \\
\hline AF2 & $1.1 \times 10^{-3}$ & $-1.2 \times 10^{-1}$ & $-3.9 \times 10^{-4}$ & $-1.3 \times 10^{-3}$ & $1.6 \times 10^{-1}$ & $3.2 \times 10^{-7}$ & $-1.5 \times 10^{1}$ & 0.20 \\
\hline $\mathrm{AF} 4$ & $9.8 \times 10^{-4}$ & $-9.4 \times 10^{-2}$ & $-6.1 \times 10^{-5}$ & $-1.2 \times 10^{-3}$ & $3.4 \times 10^{-2}$ & $3.6 \times 10^{-8}$ & $7.9 \times 10^{-1}$ & 0.21 \\
\hline AF6 & $2.0 \times 10^{-4}$ & $-7.9 \times 10^{-2}$ & $-2.7 \times 10^{-4}$ & $-1.1 \times 10^{-3}$ & $1.1 \times 10^{-1}$ & $2.3 \times 10^{-7}$ & $-8.7 \times 10^{0}$ & 0.19 \\
\hline AF8 & $4.4 \times 10^{-4}$ & $-2.7 \times 10^{-2}$ & $-1.7 \times 10^{-3}$ & $-1.8 \times 10^{-3}$ & $6.8 \times 10^{-1}$ & $1.4 \times 10^{-6}$ & $-8.3 \times 10^{1}$ & 0.24 \\
\hline AF10 & $1.9 \times 10^{-3}$ & $-8.1 \times 10^{-2}$ & $-3.8 \times 10^{-3}$ & $-2.9 \times 10^{-3}$ & $1.5 \times 10^{0}$ & $3.3 \times 10^{-6}$ & $-1.9 \times 10^{2}$ & 0.66 \\
\hline F9 & $3.5 \times 10^{-3}$ & $-3.3 \times 10^{-2}$ & $-2.8 \times 10^{-3}$ & $-2.7 \times 10^{-3}$ & $1.1 \times 10^{0}$ & $2.3 \times 10^{-6}$ & $-1.3 \times 10^{2}$ & 0.91 \\
\hline $\mathrm{F} 7$ & $-9.0 \times 10^{-3}$ & $-6.9 \times 10^{-2}$ & $5.9 \times 10^{-4}$ & $-1.8 \times 10^{-3}$ & $-2.1 \times 10^{-1}$ & $-5.2 \times 10^{-7}$ & $3.3 \times 10^{1}$ & 0.21 \\
\hline F5 & $-9.0 \times 10^{-4}$ & $-6.4 \times 10^{-2}$ & $-1.2 \times 10^{-4}$ & $-9.0 \times 10^{-4}$ & $5.0 \times 10^{-2}$ & $8.5 \times 10^{-8}$ & $-3.4 \times 10^{-1}$ & 0.09 \\
\hline F3 & $5.0 \times 10^{-4}$ & $-1.0 \times 10^{-1}$ & $-7.6 \times 10^{-4}$ & $-1.1 \times 10^{-3}$ & $2.9 \times 10^{-1}$ & $6.5 \times 10^{-7}$ & $-3.0 \times 10^{1}$ & 0.13 \\
\hline $\mathrm{F} 1$ & $6.9 \times 10^{-4}$ & $-1.1 \times 10^{-1}$ & $-6.7 \times 10^{-4}$ & $-1.1 \times 10^{-3}$ & $2.5 \times 10^{-1}$ & $6.0 \times 10^{-7}$ & $-2.3 \times 10^{1}$ & 0.13 \\
\hline $\mathrm{Fz}$ & $1.5 \times 10^{-3}$ & $-1.2 \times 10^{-1}$ & $-2.0 \times 10^{-3}$ & $-1.2 \times 10^{-3}$ & $7.9 \times 10^{-1}$ & $1.7 \times 10^{-6}$ & $-9.4 \times 10^{1}$ & 0.14 \\
\hline $\mathrm{F} 2$ & $1.4 \times 10^{-3}$ & $-9.7 \times 10^{-2}$ & $-2.0 \times 10^{-3}$ & $-1.2 \times 10^{-3}$ & $8.2 \times 10^{-1}$ & $1.7 \times 10^{-6}$ & $-1.0 \times 10^{2}$ & 0.16 \\
\hline $\mathrm{F} 4$ & $4.7 \times 10^{-4}$ & $-7.8 \times 10^{-2}$ & $-6.3 \times 10^{-4}$ & $-1.1 \times 10^{-3}$ & $2.6 \times 10^{-1}$ & $5.2 \times 10^{-7}$ & $-2.8 \times 10^{1}$ & 0.14 \\
\hline F6 & $-8.8 \times 10^{-4}$ & $-8.9 \times 10^{-2}$ & $-6.4 \times 10^{-4}$ & $-9.7 \times 10^{-4}$ & $2.6 \times 10^{-1}$ & $5.3 \times 10^{-7}$ & $-2.7 \times 10^{1}$ & 0.12 \\
\hline F8 & $-5.3 \times 10^{-4}$ & $-1.3 \times 10^{-1}$ & $1.2 \times 10^{-3}$ & $-1.8 \times 10^{-3}$ & $-4.8 \times 10^{-1}$ & $-1.1 \times 10^{-6}$ & $6.9 \times 10^{1}$ & 0.20 \\
\hline F10 & $3.3 \times 10^{-3}$ & $-3.9 \times 10^{-2}$ & $-1.5 \times 10^{-3}$ & $-2.6 \times 10^{-3}$ & $5.9 \times 10^{-1}$ & $1.2 \times 10^{-6}$ & $-7.0 \times 10^{1}$ & 0.82 \\
\hline
\end{tabular}


Table 15 (Continued).

\begin{tabular}{|c|c|c|c|c|c|c|c|c|}
\hline \multirow[b]{2}{*}{ Name } & \multicolumn{8}{|c|}{ Beta values } \\
\hline & $\beta_{0}$ & $\beta_{1}$ & $\beta_{2}$ & $\beta_{3}$ & $\beta_{4}$ & $\beta_{5}$ & $\beta_{6}$ & $R^{2}$ \\
\hline$\overline{\text { FT9 }}$ & $2.2 \times 10^{-3}$ & $6.4 \times 10^{-2}$ & $-2.7 \times 10^{-3}$ & $-2.2 \times 10^{-3}$ & $1.1 \times 10^{0}$ & $2.2 \times 10^{-6}$ & $-1.4 \times 10^{2}$ & $\overline{0.76}$ \\
\hline FT7 & $9.6 \times 10^{-4}$ & $5.0 \times 10^{-3}$ & $-1.7 \times 10^{-4}$ & $-2.1 \times 10^{-3}$ & $8.6 \times 10^{-2}$ & $1.1 \times 10^{-7}$ & $-6.8 \times 10^{0}$ & 0.26 \\
\hline FC5 & $-8.9 \times 10^{-4}$ & $-5.8 \times 10^{-2}$ & $5.2 \times 10^{-4}$ & $-1.0 \times 10^{-3}$ & $-2.1 \times 10^{-1}$ & $-4.3 \times 10^{-7}$ & $3.5 \times 10^{1}$ & 0.09 \\
\hline FC3 & $-2.6 \times 10^{-4}$ & $-1.1 \times 10^{-1}$ & $-4.0 \times 10^{-4}$ & $-1.1 \times 10^{-3}$ & $1.5 \times 10^{-1}$ & $3.4 \times 10^{-7}$ & $-1.3 \times 10^{1}$ & 0.12 \\
\hline $\mathrm{FC} 1$ & $2.0 \times 10^{-3}$ & $-1.2 \times 10^{-1}$ & $-1.3 \times 10^{-3}$ & $-1.3 \times 10^{-3}$ & $5.1 \times 10^{-1}$ & $1.1 \times 10^{-6}$ & $-5.8 \times 10^{1}$ & 0.17 \\
\hline $\mathrm{FCz}$ & $-6.6 \times 10^{-5}$ & $-1.5 \times 10^{-1}$ & $-2.1 \times 10^{-3}$ & $-1.4 \times 10^{-3}$ & $8.4 \times 10^{-1}$ & $1.8 \times 10^{-6}$ & $-1.0 \times 10^{2}$ & 0.21 \\
\hline FC2 & $2.2 \times 10^{-4}$ & $-1.2 \times 10^{-1}$ & $-4.2 \times 10^{-3}$ & $-1.4 \times 10^{-3}$ & $1.6 \times 10^{0}$ & $3.5 \times 10^{-6}$ & $-2.0 \times 10^{2}$ & 0.25 \\
\hline FC4 & $-3.6 \times 10^{-4}$ & $-1.1 \times 10^{-1}$ & $-2.4 \times 10^{-3}$ & $-1.1 \times 10^{-3}$ & $9.5 \times 10^{-1}$ & $2.0 \times 10^{-6}$ & $-1.2 \times 10^{2}$ & 0.18 \\
\hline FC6 & $-1.7 \times 10^{-3}$ & $-9.1 \times 10^{-2}$ & $-1.0 \times 10^{-3}$ & $-9.7 \times 10^{-4}$ & $4.3 \times 10^{-1}$ & $8.3 \times 10^{-7}$ & $-5.2 \times 10^{1}$ & 0.15 \\
\hline FT8 & $2.9 \times 10^{-3}$ & $2.1 \times 10^{-1}$ & $-4.0 \times 10^{-3}$ & $-1.6 \times 10^{-3}$ & $1.6 \times 10^{0}$ & $3.3 \times 10^{-6}$ & $-2.1 \times 10^{2}$ & 0.16 \\
\hline FT10 & $1.8 \times 10^{-3}$ & $9.6 \times 10^{-4}$ & $-2.3 \times 10^{-3}$ & $-2.3 \times 10^{-3}$ & $9.0 \times 10^{-1}$ & $2.0 \times 10^{-6}$ & $-1.1 \times 10^{2}$ & 0.96 \\
\hline T9 & $1.3 \times 10^{-3}$ & $-2.0 \times 10^{-2}$ & $-3.1 \times 10^{-3}$ & $-2.0 \times 10^{-3}$ & $1.2 \times 10^{0}$ & $2.6 \times 10^{-6}$ & $-1.5 \times 10^{2}$ & 0.97 \\
\hline $\mathrm{T} 7$ & $8.9 \times 10^{-4}$ & $9.6 \times 10^{-3}$ & $-5.4 \times 10^{-4}$ & $-2.1 \times 10^{-3}$ & $2.6 \times 10^{-1}$ & $3.8 \times 10^{-7}$ & $-3.3 \times 10^{1}$ & 0.25 \\
\hline $\mathrm{C} 5$ & $-1.0 \times 10^{-3}$ & $-6.1 \times 10^{-2}$ & $5.4 \times 10^{-5}$ & $-1.2 \times 10^{-3}$ & $-1.2 \times 10^{-2}$ & $-6.3 \times 10^{-8}$ & $6.9 \times 10^{0}$ & 0.13 \\
\hline $\mathrm{C} 3$ & $-7.2 \times 10^{-4}$ & $-4.6 \times 10^{-2}$ & $-4.8 \times 10^{-4}$ & $-1.4 \times 10^{-3}$ & $2.0 \times 10^{-1}$ & $3.8 \times 10^{-7}$ & $-2.2 \times 10^{1}$ & 0.20 \\
\hline $\mathrm{C} 1$ & $1.4 \times 10^{-3}$ & $-3.5 \times 10^{-2}$ & $-1.3 \times 10^{-3}$ & $-1.5 \times 10^{-3}$ & $5.1 \times 10^{-1}$ & $1.0 \times 10^{-6}$ & $-6.1 \times 10^{1}$ & 0.24 \\
\hline $\mathrm{Cz}$ & $2.6 \times 10^{-3}$ & $-1.9 \times 10^{-1}$ & $-2.8 \times 10^{-3}$ & $-1.6 \times 10^{-3}$ & $1.1 \times 10^{0}$ & $2.4 \times 10^{-6}$ & $-1.3 \times 10^{2}$ & 0.18 \\
\hline $\mathrm{C} 2$ & $1.2 \times 10^{-3}$ & $-1.6 \times 10^{-1}$ & $-3.1 \times 10^{-3}$ & $-1.5 \times 10^{-3}$ & $1.2 \times 10^{0}$ & $2.7 \times 10^{-6}$ & $-1.5 \times 10^{2}$ & 0.16 \\
\hline $\mathrm{C} 4$ & $-1.4 \times 10^{-3}$ & $-7.8 \times 10^{-2}$ & $-1.3 \times 10^{-3}$ & $-1.3 \times 10^{-3}$ & $5.2 \times 10^{-1}$ & $1.1 \times 10^{-6}$ & $-6.2 \times 10^{1}$ & 0.18 \\
\hline $\mathrm{C} 6$ & $-1.7 \times 10^{-3}$ & $-6.6 \times 10^{-2}$ & $-6.9 \times 10^{-4}$ & $-1.1 \times 10^{-3}$ & $2.9 \times 10^{-1}$ & $5.5 \times 10^{-7}$ & $-3.3 \times 10^{1}$ & 0.15 \\
\hline T8 & $-4.1 \times 10^{-3}$ & $-1.6 \times 10^{-1}$ & $-2.0 \times 10^{-3}$ & $-1.7 \times 10^{-3}$ & $7.9 \times 10^{-1}$ & $1.7 \times 10^{-6}$ & $-9.6 \times 10^{1}$ & 0.18 \\
\hline $\mathrm{T} 10$ & $2.5 \times 10^{-3}$ & $-2.7 \times 10^{-2}$ & $-4.0 \times 10^{-3}$ & $-1.8 \times 10^{-3}$ & $1.6 \times 10^{0}$ & $3.4 \times 10^{-6}$ & $-2.0 \times 10^{2}$ & 0.21 \\
\hline TP9 & $-7.0 \times 10^{-5}$ & $-5.2 \times 10^{-2}$ & $-1.8 \times 10^{-3}$ & $-1.7 \times 10^{-3}$ & $7.5 \times 10^{-1}$ & $1.4 \times 10^{-6}$ & $-9.5 \times 10^{1}$ & 0.96 \\
\hline TP7 & $-1.3 \times 10^{-3}$ & $-3.0 \times 10^{-2}$ & $5.4 \times 10^{-6}$ & $-2.3 \times 10^{-3}$ & $2.9 \times 10^{-2}$ & $-7.3 \times 10^{-8}$ & $-4.1 \times 10^{-1}$ & 0.25 \\
\hline CP5 & $-8.9 \times 10^{-4}$ & $-8.3 \times 10^{-2}$ & $8.1 \times 10^{-4}$ & $-1.5 \times 10^{-3}$ & $-2.9 \times 10^{-1}$ & $-7.4 \times 10^{-7}$ & $4.1 \times 10^{1}$ & 0.23 \\
\hline $\mathrm{CP} 3$ & $-2.6 \times 10^{-4}$ & $-4.0 \times 10^{-2}$ & $-2.7 \times 10^{-5}$ & $-1.6 \times 10^{-3}$ & $4.3 \times 10^{-2}$ & $-4.5 \times 10^{-8}$ & $-2.9 \times 10^{0}$ & 0.28 \\
\hline $\mathrm{CP} 1$ & $4.6 \times 10^{-4}$ & $-1.2 \times 10^{-3}$ & $-4.6 \times 10^{-4}$ & $-1.7 \times 10^{-3}$ & $2.1 \times 10^{-1}$ & $3.3 \times 10^{-7}$ & $-2.4 \times 10^{1}$ & 0.30 \\
\hline $\mathrm{CPz}$ & $6.3 \times 10^{-4}$ & $-1.4 \times 10^{-2}$ & $-8.1 \times 10^{-4}$ & $-1.8 \times 10^{-3}$ & $3.4 \times 10^{-1}$ & $6.4 \times 10^{-7}$ & $-4.0 \times 10^{1}$ & 0.32 \\
\hline $\mathrm{CP} 2$ & $1.8 \times 10^{-3}$ & $-7.9 \times 10^{-2}$ & $-1.4 \times 10^{-3}$ & $-1.8 \times 10^{-3}$ & $5.5 \times 10^{-1}$ & $1.2 \times 10^{-6}$ & $-6.5 \times 10^{1}$ & 0.31 \\
\hline $\mathrm{CP} 4$ & $-7.7 \times 10^{-4}$ & $-5.4 \times 10^{-2}$ & $1.5 \times 10^{-4}$ & $-1.6 \times 10^{-3}$ & $-4.1 \times 10^{-2}$ & $-1.6 \times 10^{-7}$ & $9.9 \times 10^{0}$ & 0.28 \\
\hline $\mathrm{CP} 6$ & $-1.5 \times 10^{-3}$ & $-5.7 \times 10^{-2}$ & $5.6 \times 10^{-4}$ & $-1.4 \times 10^{-3}$ & $-2.0 \times 10^{-1}$ & $-5.0 \times 10^{-7}$ & $3.0 \times 10^{1}$ & 0.17 \\
\hline TP8 & $3.4 \times 10^{-3}$ & $-1.3 \times 10^{-2}$ & $3.8 \times 10^{-4}$ & $-1.9 \times 10^{-3}$ & $-1.2 \times 10^{-1}$ & $-3.6 \times 10^{-7}$ & $2.0 \times 10^{1}$ & 0.26 \\
\hline TP10 & $-9.5 \times 10^{-4}$ & $-7.8 \times 10^{-2}$ & $-3.5 \times 10^{-3}$ & $-1.5 \times 10^{-3}$ & $1.4 \times 10^{0}$ & $2.9 \times 10^{-6}$ & $-1.8 \times 10^{2}$ & 0.54 \\
\hline
\end{tabular}


Table 15 (Continued).

\begin{tabular}{|c|c|c|c|c|c|c|c|c|}
\hline \multirow[b]{2}{*}{ Name } & \multicolumn{8}{|c|}{ Beta values } \\
\hline & $\beta_{0}$ & $\beta_{1}$ & $\beta_{2}$ & $\beta_{3}$ & $\beta_{4}$ & $\beta_{5}$ & $\beta_{6}$ & $R^{2}$ \\
\hline$\overline{P 9}$ & $8.0 \times 10^{-4}$ & $2.6 \times 10^{-2}$ & $-2.0 \times 10^{-3}$ & $-1.8 \times 10^{-3}$ & $8.1 \times 10^{-1}$ & $1.6 \times 10^{-6}$ & $-1.0 \times 10^{2}$ & $\overline{0.74}$ \\
\hline P7 & $2.3 \times 10^{-4}$ & $2.4 \times 10^{-2}$ & $-1.7 \times 10^{-3}$ & $-2.1 \times 10^{-3}$ & $6.4 \times 10^{-1}$ & $1.5 \times 10^{-6}$ & $-7.4 \times 10^{1}$ & 0.33 \\
\hline P5 & $1.3 \times 10^{-3}$ & $-7.4 \times 10^{-2}$ & $9.5 \times 10^{-4}$ & $-1.7 \times 10^{-3}$ & $-3.5 \times 10^{-1}$ & $-8.5 \times 10^{-7}$ & $5.0 \times 10^{1}$ & 0.39 \\
\hline P3 & $1.6 \times 10^{-4}$ & $-7.0 \times 10^{-2}$ & $-6.1 \times 10^{-4}$ & $-1.7 \times 10^{-3}$ & $2.7 \times 10^{-1}$ & $4.7 \times 10^{-7}$ & $-3.1 \times 10^{1}$ & 0.31 \\
\hline $\mathrm{P} 1$ & $9.0 \times 10^{-4}$ & $-3.5 \times 10^{-2}$ & $-1.2 \times 10^{-3}$ & $-1.8 \times 10^{-3}$ & $5.1 \times 10^{-1}$ & $1.0 \times 10^{-6}$ & $-6.2 \times 10^{1}$ & 0.35 \\
\hline $\mathrm{Pz}$ & $5.5 \times 10^{-4}$ & $-2.0 \times 10^{-2}$ & $-7.6 \times 10^{-4}$ & $-1.9 \times 10^{-3}$ & $3.3 \times 10^{-1}$ & $5.8 \times 10^{-7}$ & $-4.0 \times 10^{1}$ & 0.33 \\
\hline P2 & $1.0 \times 10^{-3}$ & $-3.7 \times 10^{-2}$ & $-1.1 \times 10^{-3}$ & $-1.9 \times 10^{-3}$ & $4.6 \times 10^{-1}$ & $8.9 \times 10^{-7}$ & $-5.6 \times 10^{1}$ & 0.33 \\
\hline $\mathrm{P} 4$ & $6.6 \times 10^{-4}$ & $-5.9 \times 10^{-2}$ & $-7.5 \times 10^{-4}$ & $-1.7 \times 10^{-3}$ & $3.1 \times 10^{-1}$ & $6.1 \times 10^{-7}$ & $-3.6 \times 10^{1}$ & 0.29 \\
\hline P6 & $5.2 \times 10^{-4}$ & $-6.9 \times 10^{-2}$ & $9.7 \times 10^{-4}$ & $-1.6 \times 10^{-3}$ & $-3.7 \times 10^{-1}$ & $-8.3 \times 10^{-7}$ & $5.4 \times 10^{1}$ & 0.30 \\
\hline P8 & $1.2 \times 10^{-3}$ & $6.5 \times 10^{-3}$ & $-1.7 \times 10^{-4}$ & $-2.1 \times 10^{-3}$ & $6.9 \times 10^{-2}$ & $1.6 \times 10^{-7}$ & $-2.9 \times 10^{0}$ & 0.30 \\
\hline P10 & $-1.0 \times 10^{-3}$ & $-1.1 \times 10^{-1}$ & $-3.8 \times 10^{-4}$ & $-1.7 \times 10^{-3}$ & $1.9 \times 10^{-1}$ & $2.4 \times 10^{-7}$ & $-2.3 \times 10^{1}$ & 0.86 \\
\hline PO9 & $6.1 \times 10^{-4}$ & $-7.0 \times 10^{-2}$ & $-1.8 \times 10^{-3}$ & $-1.7 \times 10^{-3}$ & $7.1 \times 10^{-1}$ & $1.5 \times 10^{-6}$ & $-8.8 \times 10^{1}$ & 0.58 \\
\hline PO7 & $-4.5 \times 10^{-3}$ & $1.2 \times 10^{-1}$ & $-2.1 \times 10^{-3}$ & $-2.3 \times 10^{-3}$ & $8.3 \times 10^{-1}$ & $1.8 \times 10^{-6}$ & $-9.9 \times 10^{1}$ & 0.31 \\
\hline PO5 & $-1.1 \times 10^{-3}$ & $-8.5 \times 10^{-2}$ & $1.0 \times 10^{-4}$ & $-1.8 \times 10^{-3}$ & $-2.1 \times 10^{-2}$ & $-1.2 \times 10^{-7}$ & $6.7 \times 10^{0}$ & 0.37 \\
\hline $\mathrm{PO} 3$ & $6.7 \times 10^{-4}$ & $-6.8 \times 10^{-2}$ & $-1.1 \times 10^{-3}$ & $-1.8 \times 10^{-3}$ & $4.6 \times 10^{-1}$ & $9.4 \times 10^{-7}$ & $-5.5 \times 10^{1}$ & 0.34 \\
\hline PO1 & $9.6 \times 10^{-4}$ & $-4.9 \times 10^{-2}$ & $-1.6 \times 10^{-3}$ & $-1.8 \times 10^{-3}$ & $6.4 \times 10^{-1}$ & $1.3 \times 10^{-6}$ & $-7.7 \times 10^{1}$ & 0.33 \\
\hline $\mathrm{POz}$ & $8.4 \times 10^{-4}$ & $-3.0 \times 10^{-2}$ & $-1.1 \times 10^{-3}$ & $-1.8 \times 10^{-3}$ & $4.7 \times 10^{-1}$ & $9.4 \times 10^{-7}$ & $-5.6 \times 10^{1}$ & 0.35 \\
\hline $\mathrm{PO} 2$ & $7.8 \times 10^{-4}$ & $-6.3 \times 10^{-2}$ & $-1.2 \times 10^{-3}$ & $-1.8 \times 10^{-3}$ & $4.9 \times 10^{-1}$ & $9.5 \times 10^{-7}$ & $-6.0 \times 10^{1}$ & 0.36 \\
\hline $\mathrm{PO} 4$ & $3.2 \times 10^{-4}$ & $-8.4 \times 10^{-2}$ & $-1.4 \times 10^{-3}$ & $-1.8 \times 10^{-3}$ & $5.6 \times 10^{-1}$ & $1.1 \times 10^{-6}$ & $-6.8 \times 10^{1}$ & 0.34 \\
\hline PO6 & $4.6 \times 10^{-4}$ & $-7.6 \times 10^{-2}$ & $1.0 \times 10^{-3}$ & $-1.9 \times 10^{-3}$ & $-3.9 \times 10^{-1}$ & $-8.7 \times 10^{-7}$ & $5.6 \times 10^{1}$ & 0.53 \\
\hline PO8 & $-1.5 \times 10^{-4}$ & $-1.2 \times 10^{-1}$ & $-2.4 \times 10^{-4}$ & $-2.3 \times 10^{-3}$ & $7.4 \times 10^{-2}$ & $2.5 \times 10^{-7}$ & $4.7 \times 10^{-2}$ & 0.53 \\
\hline P010 & $1.2 \times 10^{-3}$ & $-5.1 \times 10^{-2}$ & $-2.1 \times 10^{-3}$ & $-1.9 \times 10^{-3}$ & $8.3 \times 10^{-1}$ & $1.8 \times 10^{-6}$ & $-1.0 \times 10^{2}$ & 0.98 \\
\hline O1 & $-1.9 \times 10^{-3}$ & $4.2 \times 10^{-3}$ & $-5.0 \times 10^{-4}$ & $-2.4 \times 10^{-3}$ & $1.7 \times 10^{-1}$ & $4.6 \times 10^{-7}$ & $-1.2 \times 10^{1}$ & 0.39 \\
\hline $\mathrm{Oz}$ & $1.2 \times 10^{-3}$ & $-4.7 \times 10^{-2}$ & $1.7 \times 10^{-4}$ & $-1.9 \times 10^{-3}$ & $-5.1 \times 10^{-2}$ & $-1.7 \times 10^{-7}$ & $1.1 \times 10^{1}$ & 0.49 \\
\hline $\mathrm{O} 2$ & $-1.5 \times 10^{-3}$ & $7.4 \times 10^{-2}$ & $6.7 \times 10^{-4}$ & $-2.4 \times 10^{-3}$ & $-2.7 \times 10^{-1}$ & $-5.7 \times 10^{-7}$ & $4.0 \times 10^{1}$ & 0.36 \\
\hline 11 & $1.2 \times 10^{-3}$ & $-7.8 \times 10^{-2}$ & $-3.0 \times 10^{-3}$ & $-2.0 \times 10^{-3}$ & $1.2 \times 10^{0}$ & $2.5 \times 10^{-6}$ & $-1.5 \times 10^{2}$ & 0.89 \\
\hline $\mathrm{Iz}$ & $6.9 \times 10^{-4}$ & $-5.7 \times 10^{-2}$ & $-3.1 \times 10^{-3}$ & $-2.0 \times 10^{-3}$ & $1.2 \times 10^{0}$ & $2.5 \times 10^{-6}$ & $-1.6 \times 10^{2}$ & 0.39 \\
\hline 12 & $1.3 \times 10^{-3}$ & $-5.4 \times 10^{-2}$ & $-2.8 \times 10^{-3}$ & $-2.0 \times 10^{-3}$ & $1.1 \times 10^{0}$ & $2.3 \times 10^{-6}$ & $-1.4 \times 10^{2}$ & 0.61 \\
\hline AFp9h & $5.5 \times 10^{-3}$ & $-1.0 \times 10^{-1}$ & $-6.7 \times 10^{-3}$ & $-2.7 \times 10^{-3}$ & $2.6 \times 10^{0}$ & $5.7 \times 10^{-6}$ & $-3.3 \times 10^{2}$ & 0.44 \\
\hline AFp7h & $-1.7 \times 10^{-4}$ & $-9.4 \times 10^{-2}$ & $-7.2 \times 10^{-4}$ & $-1.4 \times 10^{-3}$ & $3.1 \times 10^{-1}$ & $5.7 \times 10^{-7}$ & $-3.6 \times 10^{1}$ & 0.19 \\
\hline AFp5h & $3.1 \times 10^{-4}$ & $-8.0 \times 10^{-2}$ & $2.6 \times 10^{-4}$ & $-1.1 \times 10^{-3}$ & $-8.1 \times 10^{-2}$ & $-2.5 \times 10^{-7}$ & $1.4 \times 10^{1}$ & 0.18 \\
\hline AFp3h & $1.2 \times 10^{-3}$ & $-9.4 \times 10^{-2}$ & $3.7 \times 10^{-4}$ & $-1.2 \times 10^{-3}$ & $-1.4 \times 10^{-1}$ & $-3.3 \times 10^{-7}$ & $2.3 \times 10^{1}$ & 0.18 \\
\hline AFp1h & $2.1 \times 10^{-3}$ & $-1.2 \times 10^{-1}$ & $1.6 \times 10^{-5}$ & $-1.4 \times 10^{-3}$ & $-5.6 \times 10^{-3}$ & $-1.2 \times 10^{-8}$ & $7.5 \times 10^{0}$ & 0.18 \\
\hline
\end{tabular}


Table 15 (Continued).

\begin{tabular}{|c|c|c|c|c|c|c|c|c|}
\hline \multirow[b]{2}{*}{ Name } & \multicolumn{8}{|c|}{ Beta values } \\
\hline & $\beta_{0}$ & $\beta_{1}$ & $\beta_{2}$ & $\beta_{3}$ & $\beta_{4}$ & $\beta_{5}$ & $\beta_{6}$ & $R^{2}$ \\
\hline AFp2h & $1.6 \times 10^{-3}$ & $-1.1 \times 10^{-1}$ & $8.9 \times 10^{-5}$ & $-1.5 \times 10^{-3}$ & $-3.6 \times 10^{-2}$ & $-7.1 \times 10^{-8}$ & $1.2 \times 10^{1}$ & 0.18 \\
\hline AFp4h & $6.9 \times 10^{-4}$ & $-9.1 \times 10^{-2}$ & $3.6 \times 10^{-4}$ & $-1.4 \times 10^{-3}$ & $-1.3 \times 10^{-1}$ & $-3.2 \times 10^{-7}$ & $2.2 \times 10^{1}$ & 0.19 \\
\hline AFp6h & $3.7 \times 10^{-4}$ & $-7.2 \times 10^{-2}$ & $4.7 \times 10^{-4}$ & $-1.4 \times 10^{-3}$ & $-1.7 \times 10^{-1}$ & $-4.2 \times 10^{-7}$ & $2.7 \times 10^{1}$ & 0.21 \\
\hline AFp8h & $4.4 \times 10^{-3}$ & $-5.7 \times 10^{-2}$ & $6.6 \times 10^{-4}$ & $-1.6 \times 10^{-3}$ & $-2.7 \times 10^{-1}$ & $-5.3 \times 10^{-7}$ & $4.4 \times 10^{1}$ & 0.20 \\
\hline AFp10h & $1.4 \times 10^{-3}$ & $3.5 \times 10^{-2}$ & $-8.3 \times 10^{-4}$ & $-2.8 \times 10^{-3}$ & $3.3 \times 10^{-1}$ & $6.6 \times 10^{-7}$ & $-3.1 \times 10^{1}$ & 0.39 \\
\hline AFF9h & $3.3 \times 10^{-3}$ & $-2.3 \times 10^{-2}$ & $-5.9 \times 10^{-3}$ & $-3.2 \times 10^{-3}$ & $2.3 \times 10^{0}$ & $4.9 \times 10^{-6}$ & $-3.0 \times 10^{2}$ & 0.26 \\
\hline AFF7h & $3.6 \times 10^{-5}$ & $-1.2 \times 10^{-1}$ & $-8.9 \times 10^{-4}$ & $-9.2 \times 10^{-4}$ & $3.7 \times 10^{-1}$ & $7.1 \times 10^{-7}$ & $-4.5 \times 10^{1}$ & 0.21 \\
\hline AFF5h & $7.0 \times 10^{-4}$ & $-1.0 \times 10^{-1}$ & $-9.9 \times 10^{-5}$ & $-9.9 \times 10^{-4}$ & $4.9 \times 10^{-2}$ & $6.3 \times 10^{-8}$ & $-1.0 \times 10^{0}$ & 0.15 \\
\hline AFF3h & $6.1 \times 10^{-4}$ & $-1.0 \times 10^{-1}$ & $-1.7 \times 10^{-4}$ & $-1.1 \times 10^{-3}$ & $7.1 \times 10^{-2}$ & $1.4 \times 10^{-7}$ & $-2.7 \times 10^{0}$ & 0.15 \\
\hline AFF1h & $1.2 \times 10^{-3}$ & $-1.3 \times 10^{-1}$ & $-4.0 \times 10^{-4}$ & $-1.2 \times 10^{-3}$ & $1.6 \times 10^{-1}$ & $3.4 \times 10^{-7}$ & $-1.4 \times 10^{1}$ & 0.17 \\
\hline AFF2h & $1.6 \times 10^{-3}$ & $-1.3 \times 10^{-1}$ & $-1.3 \times 10^{-3}$ & $-1.3 \times 10^{-3}$ & $5.2 \times 10^{-1}$ & $1.1 \times 10^{-6}$ & $-6.1 \times 10^{1}$ & 0.19 \\
\hline AFF4h & $1.1 \times 10^{-3}$ & $-1.1 \times 10^{-1}$ & $-6.1 \times 10^{-4}$ & $-1.2 \times 10^{-3}$ & $2.5 \times 10^{-1}$ & $4.9 \times 10^{-7}$ & $-2.8 \times 10^{1}$ & 0.19 \\
\hline AFF6h & $-6.5 \times 10^{-4}$ & $-1.2 \times 10^{-1}$ & $-1.1 \times 10^{-3}$ & $-1.0 \times 10^{-3}$ & $4.4 \times 10^{-1}$ & $9.6 \times 10^{-7}$ & $-5.1 \times 10^{1}$ & 0.15 \\
\hline AFF8h & $-1.4 \times 10^{-3}$ & $-1.4 \times 10^{-1}$ & $-5.5 \times 10^{-4}$ & $-9.8 \times 10^{-4}$ & $2.3 \times 10^{-1}$ & $4.5 \times 10^{-7}$ & $-2.4 \times 10^{1}$ & 0.15 \\
\hline AFF10h & $4.7 \times 10^{-3}$ & $-1.8 \times 10^{-1}$ & $-4.7 \times 10^{-3}$ & $-3.0 \times 10^{-3}$ & $1.8 \times 10^{0}$ & $3.9 \times 10^{-6}$ & $-2.3 \times 10^{2}$ & 0.30 \\
\hline FFT9h & $1.0 \times 10^{-3}$ & $1.2 \times 10^{-2}$ & $-1.8 \times 10^{-3}$ & $-2.6 \times 10^{-3}$ & $7.0 \times 10^{-1}$ & $1.5 \times 10^{-6}$ & $-8.5 \times 10^{1}$ & 0.28 \\
\hline FFT7h & $-2.3 \times 10^{-3}$ & $-2.5 \times 10^{-2}$ & $7.0 \times 10^{-4}$ & $-1.1 \times 10^{-3}$ & $-2.6 \times 10^{-1}$ & $-6.1 \times 10^{-7}$ & $4.0 \times 10^{1}$ & 0.19 \\
\hline FFC5h & $-3.3 \times 10^{-4}$ & $-8.3 \times 10^{-2}$ & $-3.6 \times 10^{-4}$ & $-9.8 \times 10^{-4}$ & $1.3 \times 10^{-1}$ & $3.2 \times 10^{-7}$ & $-9.5 \times 10^{0}$ & 0.10 \\
\hline FFC3h & $8.8 \times 10^{-4}$ & $-1.1 \times 10^{-1}$ & $-7.7 \times 10^{-4}$ & $-1.2 \times 10^{-3}$ & $2.8 \times 10^{-1}$ & $6.8 \times 10^{-7}$ & $-2.8 \times 10^{1}$ & 0.14 \\
\hline FFC1h & $1.2 \times 10^{-3}$ & $-1.1 \times 10^{-1}$ & $-1.0 \times 10^{-3}$ & $-1.2 \times 10^{-3}$ & $3.8 \times 10^{-1}$ & $9.3 \times 10^{-7}$ & $-4.0 \times 10^{1}$ & 0.13 \\
\hline FFC2h & $9.4 \times 10^{-4}$ & $-1.3 \times 10^{-1}$ & $-3.1 \times 10^{-3}$ & $-1.3 \times 10^{-3}$ & $1.2 \times 10^{0}$ & $2.7 \times 10^{-6}$ & $-1.5 \times 10^{2}$ & 0.24 \\
\hline FFC4h & $7.8 \times 10^{-4}$ & $-8.5 \times 10^{-2}$ & $-2.2 \times 10^{-3}$ & $-1.1 \times 10^{-3}$ & $8.6 \times 10^{-1}$ & $1.8 \times 10^{-6}$ & $-1.1 \times 10^{2}$ & 0.24 \\
\hline FFC6h & $-2.6 \times 10^{-4}$ & $-7.9 \times 10^{-2}$ & $-1.2 \times 10^{-3}$ & $-1.0 \times 10^{-3}$ & $4.7 \times 10^{-1}$ & $9.6 \times 10^{-7}$ & $-5.7 \times 10^{1}$ & 0.14 \\
\hline FFT8h & $-2.6 \times 10^{-3}$ & $3.3 \times 10^{-3}$ & $2.6 \times 10^{-4}$ & $-1.1 \times 10^{-3}$ & $-6.8 \times 10^{-2}$ & $-2.7 \times 10^{-7}$ & $1.1 \times 10^{1}$ & 0.15 \\
\hline FFT10h & $3.1 \times 10^{-3}$ & $9.7 \times 10^{-2}$ & $-4.0 \times 10^{-3}$ & $-2.5 \times 10^{-3}$ & $1.6 \times 10^{0}$ & $3.4 \times 10^{-6}$ & $-2.0 \times 10^{2}$ & 0.24 \\
\hline FTT9h & $4.4 \times 10^{-3}$ & $3.6 \times 10^{-2}$ & $-3.9 \times 10^{-3}$ & $-2.6 \times 10^{-3}$ & $1.5 \times 10^{0}$ & $3.3 \times 10^{-6}$ & $-1.9 \times 10^{2}$ & 0.29 \\
\hline FTT7h & $-1.6 \times 10^{-4}$ & $-4.1 \times 10^{-2}$ & $3.2 \times 10^{-4}$ & $-1.5 \times 10^{-3}$ & $-1.3 \times 10^{-1}$ & $-2.6 \times 10^{-7}$ & $2.4 \times 10^{1}$ & 0.16 \\
\hline FCC5h & $-1.0 \times 10^{-3}$ & $-6.3 \times 10^{-2}$ & $1.9 \times 10^{-4}$ & $-1.1 \times 10^{-3}$ & $-7.7 \times 10^{-2}$ & $-1.6 \times 10^{-7}$ & $1.7 \times 10^{1}$ & 0.10 \\
\hline FCC3h & $2.7 \times 10^{-5}$ & $-8.1 \times 10^{-2}$ & $-6.6 \times 10^{-4}$ & $-1.4 \times 10^{-3}$ & $2.7 \times 10^{-1}$ & $5.4 \times 10^{-7}$ & $-3.0 \times 10^{1}$ & 0.21 \\
\hline FCC1h & $2.1 \times 10^{-3}$ & $-1.4 \times 10^{-1}$ & $-2.2 \times 10^{-3}$ & $-1.4 \times 10^{-3}$ & $8.8 \times 10^{-1}$ & $1.9 \times 10^{-6}$ & $-1.1 \times 10^{2}$ & 0.21 \\
\hline $\mathrm{FCC} 2 \mathrm{~h}$ & $5.3 \times 10^{-4}$ & $-2.4 \times 10^{-1}$ & $-4.4 \times 10^{-3}$ & $-1.5 \times 10^{-3}$ & $1.7 \times 10^{0}$ & $3.8 \times 10^{-6}$ & $-2.1 \times 10^{2}$ & 0.18 \\
\hline FCC4h & $-1.5 \times 10^{-3}$ & $-1.1 \times 10^{-1}$ & $-3.3 \times 10^{-3}$ & $-1.3 \times 10^{-3}$ & $1.3 \times 10^{0}$ & $2.8 \times 10^{-6}$ & $-1.6 \times 10^{2}$ & 0.17 \\
\hline FCC6h & $-1.2 \times 10^{-3}$ & $-8.3 \times 10^{-2}$ & $-1.3 \times 10^{-3}$ & $-1.0 \times 10^{-3}$ & $5.3 \times 10^{-1}$ & $1.1 \times 10^{-6}$ & $-6.4 \times 10^{1}$ & 0.14 \\
\hline
\end{tabular}


Table 15 (Continued).

\begin{tabular}{|c|c|c|c|c|c|c|c|c|}
\hline \multirow[b]{2}{*}{ Name } & \multicolumn{8}{|c|}{ Beta values } \\
\hline & $\beta_{0}$ & $\beta_{1}$ & $\beta_{2}$ & $\beta_{3}$ & $\beta_{4}$ & $\beta_{5}$ & $\beta_{6}$ & $R^{2}$ \\
\hline FTT8h & $-2.5 \times 10^{-3}$ & $-8.4 \times 10^{-2}$ & $-2.0 \times 10^{-3}$ & $-1.1 \times 10^{-3}$ & $8.1 \times 10^{-1}$ & $1.6 \times 10^{-6}$ & $-1.0 \times 10^{2}$ & $\overline{0.12}$ \\
\hline FTT10h & $-1.8 \times 10^{-3}$ & $-4.1 \times 10^{-2}$ & $-2.4 \times 10^{-3}$ & $-1.9 \times 10^{-3}$ & $9.9 \times 10^{-1}$ & $2.0 \times 10^{-6}$ & $-1.3 \times 10^{2}$ & 0.18 \\
\hline TTP9h & $2.0 \times 10^{-3}$ & $-6.7 \times 10^{-3}$ & $-1.5 \times 10^{-3}$ & $-2.1 \times 10^{-3}$ & $6.0 \times 10^{-1}$ & $1.3 \times 10^{-6}$ & $-7.0 \times 10^{1}$ & 0.15 \\
\hline TTP7h & $-5.3 \times 10^{-4}$ & $-1.1 \times 10^{-1}$ & $2.1 \times 10^{-3}$ & $-1.5 \times 10^{-3}$ & $-8.1 \times 10^{-1}$ & $-1.9 \times 10^{-6}$ & $1.1 \times 10^{2}$ & 0.26 \\
\hline CCP5h & $-9.6 \times 10^{-4}$ & $-5.1 \times 10^{-2}$ & $-2.3 \times 10^{-4}$ & $-1.3 \times 10^{-3}$ & $1.1 \times 10^{-1}$ & $1.5 \times 10^{-7}$ & $-1.1 \times 10^{1}$ & 0.17 \\
\hline ССР3h & $2.7 \times 10^{-4}$ & $-1.8 \times 10^{-2}$ & $-4.3 \times 10^{-4}$ & $-1.7 \times 10^{-3}$ & $1.9 \times 10^{-1}$ & $3.1 \times 10^{-7}$ & $-2.1 \times 10^{1}$ & 0.26 \\
\hline CCP1h & $1.3 \times 10^{-3}$ & $-5.0 \times 10^{-4}$ & $-1.1 \times 10^{-3}$ & $-1.6 \times 10^{-3}$ & $4.4 \times 10^{-1}$ & $9.1 \times 10^{-7}$ & $-5.2 \times 10^{1}$ & 0.24 \\
\hline CCP2h & $3.4 \times 10^{-3}$ & $-1.7 \times 10^{-1}$ & $-1.9 \times 10^{-3}$ & $-1.7 \times 10^{-3}$ & $7.3 \times 10^{-1}$ & $1.7 \times 10^{-6}$ & $-8.5 \times 10^{1}$ & 0.18 \\
\hline CCP4h & $-4.2 \times 10^{-4}$ & $-6.6 \times 10^{-2}$ & $-1.2 \times 10^{-3}$ & $-1.5 \times 10^{-3}$ & $4.9 \times 10^{-1}$ & $1.0 \times 10^{-6}$ & $-5.9 \times 10^{1}$ & 0.26 \\
\hline CCP6h & $-2.1 \times 10^{-3}$ & $-6.1 \times 10^{-2}$ & $-2.6 \times 10^{-4}$ & $-1.3 \times 10^{-3}$ & $1.2 \times 10^{-1}$ & $1.9 \times 10^{-7}$ & $-1.1 \times 10^{1}$ & 0.20 \\
\hline TTP8h & $8.8 \times 10^{-4}$ & $1.1 \times 10^{-2}$ & $8.0 \times 10^{-4}$ & $-1.4 \times 10^{-3}$ & $-2.8 \times 10^{-1}$ & $-7.4 \times 10^{-7}$ & $3.8 \times 10^{1}$ & 0.19 \\
\hline TTP10h & $1.6 \times 10^{-3}$ & $-4.6 \times 10^{-2}$ & $-2.7 \times 10^{-3}$ & $-2.3 \times 10^{-3}$ & $1.1 \times 10^{0}$ & $2.3 \times 10^{-6}$ & $-1.4 \times 10^{2}$ & 0.24 \\
\hline TPP9h & $-1.2 \times 10^{-4}$ & $-4.0 \times 10^{-4}$ & $-8.9 \times 10^{-4}$ & $-1.9 \times 10^{-3}$ & $3.7 \times 10^{-1}$ & $7.1 \times 10^{-7}$ & $-4.4 \times 10^{1}$ & 0.20 \\
\hline TPP7h & $4.4 \times 10^{-4}$ & $-1.1 \times 10^{-1}$ & $6.5 \times 10^{-4}$ & $-1.8 \times 10^{-3}$ & $-2.3 \times 10^{-1}$ & $-5.8 \times 10^{-7}$ & $3.4 \times 10^{1}$ & 0.30 \\
\hline CPP5h & $-3.8 \times 10^{-4}$ & $-6.6 \times 10^{-2}$ & $1.6 \times 10^{-4}$ & $-1.6 \times 10^{-3}$ & $-3.0 \times 10^{-2}$ & $-2.0 \times 10^{-7}$ & $6.5 \times 10^{0}$ & 0.27 \\
\hline СPP3h & $1.8 \times 10^{-4}$ & $-4.4 \times 10^{-2}$ & $-4.8 \times 10^{-4}$ & $-1.7 \times 10^{-3}$ & $2.2 \times 10^{-1}$ & $3.6 \times 10^{-7}$ & $-2.5 \times 10^{1}$ & 0.33 \\
\hline CPP1h & $7.6 \times 10^{-4}$ & $-4.4 \times 10^{-3}$ & $-6.0 \times 10^{-4}$ & $-1.8 \times 10^{-3}$ & $2.7 \times 10^{-1}$ & $4.5 \times 10^{-7}$ & $-3.2 \times 10^{1}$ & 0.34 \\
\hline CPP2h & $6.3 \times 10^{-4}$ & $-2.4 \times 10^{-2}$ & $-9.8 \times 10^{-4}$ & $-1.9 \times 10^{-3}$ & $4.1 \times 10^{-1}$ & $7.8 \times 10^{-7}$ & $-5.0 \times 10^{1}$ & 0.32 \\
\hline CPP4h & $1.8 \times 10^{-4}$ & $-4.7 \times 10^{-2}$ & $-7.4 \times 10^{-4}$ & $-1.8 \times 10^{-3}$ & $3.1 \times 10^{-1}$ & $5.9 \times 10^{-7}$ & $-3.6 \times 10^{1}$ & 0.33 \\
\hline CPP6h & $-2.3 \times 10^{-4}$ & $-6.6 \times 10^{-2}$ & $8.5 \times 10^{-4}$ & $-1.6 \times 10^{-3}$ & $-3.2 \times 10^{-1}$ & $-7.4 \times 10^{-7}$ & $4.7 \times 10^{1}$ & 0.23 \\
\hline TPP8h & $-4.6 \times 10^{-4}$ & $-4.1 \times 10^{-2}$ & $1.2 \times 10^{-3}$ & $-1.6 \times 10^{-3}$ & $-4.5 \times 10^{-1}$ & $-1.0 \times 10^{-6}$ & $6.4 \times 10^{1}$ & 0.25 \\
\hline TPP10h & $-2.1 \times 10^{-4}$ & $1.1 \times 10^{-2}$ & $-1.5 \times 10^{-4}$ & $-1.8 \times 10^{-3}$ & $7.0 \times 10^{-2}$ & $8.7 \times 10^{-8}$ & $-4.1 \times 10^{0}$ & 0.21 \\
\hline PPO9h & $9.3 \times 10^{-4}$ & $-5.7 \times 10^{-2}$ & $-1.5 \times 10^{-3}$ & $-2.2 \times 10^{-3}$ & $6.0 \times 10^{-1}$ & $1.2 \times 10^{-6}$ & $-7.1 \times 10^{1}$ & 0.37 \\
\hline PPO7h & $-4.2 \times 10^{-4}$ & $-1.7 \times 10^{-2}$ & $1.9 \times 10^{-3}$ & $-2.1 \times 10^{-3}$ & $-7.4 \times 10^{-1}$ & $-1.6 \times 10^{-6}$ & $1.0 \times 10^{2}$ & 0.41 \\
\hline PPO5h & $2.0 \times 10^{-4}$ & $-9.3 \times 10^{-2}$ & $-6.5 \times 10^{-4}$ & $-1.7 \times 10^{-3}$ & $2.8 \times 10^{-1}$ & $5.0 \times 10^{-7}$ & $-3.3 \times 10^{1}$ & 0.32 \\
\hline PPO3h & $8.2 \times 10^{-4}$ & $-5.9 \times 10^{-2}$ & $-1.8 \times 10^{-3}$ & $-1.8 \times 10^{-3}$ & $7.2 \times 10^{-1}$ & $1.5 \times 10^{-6}$ & $-8.9 \times 10^{1}$ & 0.32 \\
\hline PPO1h & $6.6 \times 10^{-4}$ & $-3.6 \times 10^{-2}$ & $-1.6 \times 10^{-3}$ & $-1.8 \times 10^{-3}$ & $6.5 \times 10^{-1}$ & $1.3 \times 10^{-6}$ & $-8.0 \times 10^{1}$ & 0.33 \\
\hline PPO2h & $7.2 \times 10^{-4}$ & $-3.4 \times 10^{-2}$ & $-9.6 \times 10^{-4}$ & $-1.8 \times 10^{-3}$ & $4.1 \times 10^{-1}$ & $7.6 \times 10^{-7}$ & $-5.0 \times 10^{1}$ & 0.33 \\
\hline PPO4h & $9.0 \times 10^{-4}$ & $-6.8 \times 10^{-2}$ & $-1.2 \times 10^{-3}$ & $-1.9 \times 10^{-3}$ & $4.7 \times 10^{-1}$ & $9.4 \times 10^{-7}$ & $-5.7 \times 10^{1}$ & 0.32 \\
\hline PPO6h & $7.7 \times 10^{-4}$ & $-6.9 \times 10^{-2}$ & $-2.6 \times 10^{-4}$ & $-1.8 \times 10^{-3}$ & $1.2 \times 10^{-1}$ & $2.0 \times 10^{-7}$ & $-1.0 \times 10^{1}$ & 0.33 \\
\hline PPO8h & $1.3 \times 10^{-3}$ & $-9.1 \times 10^{-2}$ & $9.1 \times 10^{-4}$ & $-1.7 \times 10^{-3}$ & $-3.6 \times 10^{-1}$ & $-7.7 \times 10^{-7}$ & $5.5 \times 10^{1}$ & 0.37 \\
\hline PPO10h & $-2.6 \times 10^{-3}$ & $-6.2 \times 10^{-2}$ & $-1.0 \times 10^{-3}$ & $-2.3 \times 10^{-3}$ & $4.2 \times 10^{-1}$ & $8.6 \times 10^{-7}$ & $-4.8 \times 10^{1}$ & 0.25 \\
\hline POO9h & $8.5 \times 10^{-4}$ & $6.7 \times 10^{-3}$ & $-1.5 \times 10^{-3}$ & $-2.1 \times 10^{-3}$ & $6.1 \times 10^{-1}$ & $1.2 \times 10^{-6}$ & $-7.7 \times 10^{1}$ & 0.37 \\
\hline
\end{tabular}


Table 15 (Continued).

\begin{tabular}{|c|c|c|c|c|c|c|c|c|}
\hline \multirow[b]{2}{*}{ Name } & \multicolumn{8}{|c|}{ Beta values } \\
\hline & $\beta_{0}$ & $\beta_{1}$ & $\beta_{2}$ & $\beta_{3}$ & $\beta_{4}$ & $\beta_{5}$ & $\beta_{6}$ & $R^{2}$ \\
\hline POO7h & $-4.8 \times 10^{-6}$ & $-5.2 \times 10^{-2}$ & $1.2 \times 10^{-3}$ & $-2.2 \times 10^{-3}$ & $-4.8 \times 10^{-1}$ & $-1.0 \times 10^{-6}$ & $7.0 \times 10^{1}$ & $\overline{0.45}$ \\
\hline POO5h & $-1.0 \times 10^{-3}$ & $-1.1 \times 10^{-2}$ & $-9.4 \times 10^{-4}$ & $-2.0 \times 10^{-3}$ & $3.8 \times 10^{-1}$ & $7.8 \times 10^{-7}$ & $-4.5 \times 10^{1}$ & 0.34 \\
\hline POO3h & $-2.7 \times 10^{-4}$ & $-9.4 \times 10^{-2}$ & $-1.6 \times 10^{-4}$ & $-1.8 \times 10^{-3}$ & $6.7 \times 10^{-2}$ & $1.4 \times 10^{-7}$ & $-2.1 \times 10^{0}$ & 0.34 \\
\hline P001h & $1.4 \times 10^{-3}$ & $-8.7 \times 10^{-2}$ & $-1.6 \times 10^{-3}$ & $-1.9 \times 10^{-3}$ & $6.4 \times 10^{-1}$ & $1.3 \times 10^{-6}$ & $-7.8 \times 10^{1}$ & 0.47 \\
\hline POO2h & $1.6 \times 10^{-3}$ & $-6.2 \times 10^{-2}$ & $-5.8 \times 10^{-4}$ & $-1.8 \times 10^{-3}$ & $2.4 \times 10^{-1}$ & $4.7 \times 10^{-7}$ & $-2.7 \times 10^{1}$ & 0.38 \\
\hline POO4h & $7.6 \times 10^{-4}$ & $-8.1 \times 10^{-2}$ & $-8.9 \times 10^{-4}$ & $-1.8 \times 10^{-3}$ & $3.7 \times 10^{-1}$ & $7.1 \times 10^{-7}$ & $-4.4 \times 10^{1}$ & 0.39 \\
\hline POO6h & $-3.5 \times 10^{-4}$ & $-9.6 \times 10^{-2}$ & $3.4 \times 10^{-4}$ & $-1.9 \times 10^{-3}$ & $-1.2 \times 10^{-1}$ & $-3.1 \times 10^{-7}$ & $2.0 \times 10^{1}$ & 0.42 \\
\hline POO8h & $-1.4 \times 10^{-3}$ & $-3.6 \times 10^{-2}$ & $-8.9 \times 10^{-4}$ & $-2.0 \times 10^{-3}$ & $3.5 \times 10^{-1}$ & $7.5 \times 10^{-7}$ & $-3.9 \times 10^{1}$ & 0.31 \\
\hline PoO10h & $2.5 \times 10^{-3}$ & $-7.8 \times 10^{-2}$ & $-2.7 \times 10^{-3}$ & $-2.2 \times 10^{-3}$ & $1.1 \times 10^{0}$ & $2.3 \times 10^{-6}$ & $-1.3 \times 10^{2}$ & 0.38 \\
\hline Ol1h & $7.2 \times 10^{-4}$ & $-1.1 \times 10^{-1}$ & $-3.8 \times 10^{-3}$ & $-2.3 \times 10^{-3}$ & $1.5 \times 10^{0}$ & $3.2 \times 10^{-6}$ & $-1.9 \times 10^{2}$ & 0.40 \\
\hline $\mathrm{OI} 2 \mathrm{~h}$ & $7.6 \times 10^{-4}$ & $-1.2 \times 10^{-1}$ & $-4.0 \times 10^{-3}$ & $-2.1 \times 10^{-3}$ & $1.6 \times 10^{0}$ & $3.4 \times 10^{-6}$ & $-1.9 \times 10^{2}$ & 0.36 \\
\hline Fp1h & $3.1 \times 10^{-3}$ & $-1.6 \times 10^{-1}$ & $8.1 \times 10^{-4}$ & $-1.4 \times 10^{-3}$ & $-3.3 \times 10^{-1}$ & $-6.6 \times 10^{-7}$ & $5.2 \times 10^{1}$ & 0.14 \\
\hline $\mathrm{Fp} 2 \mathrm{~h}$ & $3.1 \times 10^{-3}$ & $-1.7 \times 10^{-1}$ & $3.1 \times 10^{-4}$ & $-1.4 \times 10^{-3}$ & $-1.3 \times 10^{-1}$ & $-2.6 \times 10^{-7}$ & $2.3 \times 10^{1}$ & 0.16 \\
\hline AF9h & $2.2 \times 10^{-3}$ & $-6.2 \times 10^{-2}$ & $-3.4 \times 10^{-3}$ & $-3.2 \times 10^{-3}$ & $1.4 \times 10^{0}$ & $2.9 \times 10^{-6}$ & $-1.7 \times 10^{2}$ & 0.37 \\
\hline AF7h & $1.6 \times 10^{-3}$ & $-1.8 \times 10^{-1}$ & $-2.2 \times 10^{-4}$ & $-9.8 \times 10^{-4}$ & $1.1 \times 10^{-1}$ & $1.6 \times 10^{-7}$ & $-9.5 \times 10^{0}$ & 0.21 \\
\hline AF5h & $1.0 \times 10^{-3}$ & $-1.0 \times 10^{-1}$ & $1.1 \times 10^{-4}$ & $-1.1 \times 10^{-3}$ & $-3.2 \times 10^{-2}$ & $-1.2 \times 10^{-7}$ & $9.2 \times 10^{0}$ & 0.17 \\
\hline AF3h & $1.1 \times 10^{-3}$ & $-1.0 \times 10^{-1}$ & $1.5 \times 10^{-4}$ & $-1.1 \times 10^{-3}$ & $-4.8 \times 10^{-2}$ & $-1.5 \times 10^{-7}$ & $1.1 \times 10^{1}$ & 0.18 \\
\hline AF1h & $1.7 \times 10^{-3}$ & $-1.2 \times 10^{-1}$ & $-3.5 \times 10^{-4}$ & $-1.3 \times 10^{-3}$ & $1.4 \times 10^{-1}$ & $2.9 \times 10^{-7}$ & $-1.1 \times 10^{1}$ & 0.18 \\
\hline AF2h & $1.3 \times 10^{-3}$ & $-1.1 \times 10^{-1}$ & $-6.2 \times 10^{-4}$ & $-1.4 \times 10^{-3}$ & $2.5 \times 10^{-1}$ & $5.2 \times 10^{-7}$ & $-2.6 \times 10^{1}$ & 0.19 \\
\hline $\mathrm{AF} 4 \mathrm{~h}$ & $1.1 \times 10^{-3}$ & $-1.1 \times 10^{-1}$ & $-1.5 \times 10^{-4}$ & $-1.3 \times 10^{-3}$ & $6.8 \times 10^{-2}$ & $1.1 \times 10^{-7}$ & $-3.6 \times 10^{0}$ & 0.21 \\
\hline AF6h & $7.0 \times 10^{-4}$ & $-7.7 \times 10^{-2}$ & $1.6 \times 10^{-5}$ & $-1.1 \times 10^{-3}$ & $2.8 \times 10^{-3}$ & $-2.7 \times 10^{-8}$ & $4.9 \times 10^{0}$ & 0.21 \\
\hline AF8h & $4.5 \times 10^{-5}$ & $-1.1 \times 10^{-1}$ & $-1.3 \times 10^{-3}$ & $-1.1 \times 10^{-3}$ & $5.0 \times 10^{-1}$ & $1.1 \times 10^{-6}$ & $-5.9 \times 10^{1}$ & 0.17 \\
\hline AF10h & $2.5 \times 10^{-3}$ & $-4.2 \times 10^{-2}$ & $-2.5 \times 10^{-3}$ & $-3.3 \times 10^{-3}$ & $9.7 \times 10^{-1}$ & $2.1 \times 10^{-6}$ & $-1.2 \times 10^{2}$ & 0.41 \\
\hline F9h & $1.7 \times 10^{-3}$ & $-7.1 \times 10^{-2}$ & $-3.8 \times 10^{-3}$ & $-2.9 \times 10^{-3}$ & $1.5 \times 10^{0}$ & $3.2 \times 10^{-6}$ & $-1.9 \times 10^{2}$ & 0.32 \\
\hline $\mathrm{F} 7 \mathrm{~h}$ & $-4.2 \times 10^{-3}$ & $-2.9 \times 10^{-2}$ & $-2.0 \times 10^{-4}$ & $-1.1 \times 10^{-3}$ & $9.5 \times 10^{-2}$ & $1.4 \times 10^{-7}$ & $-7.7 \times 10^{0}$ & 0.15 \\
\hline $\mathrm{F} 5 \mathrm{~h}$ & $3.1 \times 10^{-5}$ & $-9.1 \times 10^{-2}$ & $-5.7 \times 10^{-4}$ & $-9.8 \times 10^{-4}$ & $2.2 \times 10^{-1}$ & $4.8 \times 10^{-7}$ & $-2.2 \times 10^{1}$ & 0.11 \\
\hline F3h & $5.9 \times 10^{-4}$ & $-1.1 \times 10^{-1}$ & $-6.4 \times 10^{-4}$ & $-1.2 \times 10^{-3}$ & $2.4 \times 10^{-1}$ & $5.7 \times 10^{-7}$ & $-2.3 \times 10^{1}$ & 0.13 \\
\hline $\mathrm{F} 1 \mathrm{~h}$ & $1.1 \times 10^{-3}$ & $-1.2 \times 10^{-1}$ & $-1.1 \times 10^{-3}$ & $-1.1 \times 10^{-3}$ & $4.0 \times 10^{-1}$ & $9.4 \times 10^{-7}$ & $-4.2 \times 10^{1}$ & 0.14 \\
\hline $\mathrm{F} 2 \mathrm{~h}$ & $1.7 \times 10^{-3}$ & $-1.1 \times 10^{-1}$ & $-2.5 \times 10^{-3}$ & $-1.2 \times 10^{-3}$ & $9.8 \times 10^{-1}$ & $2.1 \times 10^{-6}$ & $-1.2 \times 10^{2}$ & 0.16 \\
\hline $\mathrm{F} 4 \mathrm{~h}$ & $1.1 \times 10^{-3}$ & $-8.0 \times 10^{-2}$ & $-1.2 \times 10^{-3}$ & $-1.1 \times 10^{-3}$ & $4.9 \times 10^{-1}$ & $9.8 \times 10^{-7}$ & $-5.9 \times 10^{1}$ & 0.16 \\
\hline F6h & $-3.9 \times 10^{-4}$ & $-7.2 \times 10^{-2}$ & $-5.5 \times 10^{-4}$ & $-1.0 \times 10^{-3}$ & $2.3 \times 10^{-1}$ & $4.5 \times 10^{-7}$ & $-2.4 \times 10^{1}$ & 0.11 \\
\hline F8h & $-1.6 \times 10^{-3}$ & $-7.4 \times 10^{-2}$ & $8.0 \times 10^{-4}$ & $-1.1 \times 10^{-3}$ & $-3.0 \times 10^{-1}$ & $-7.0 \times 10^{-7}$ & $4.5 \times 10^{1}$ & 0.16 \\
\hline F10h & $5.4 \times 10^{-3}$ & $-2.0 \times 10^{-2}$ & $-1.2 \times 10^{-3}$ & $-2.9 \times 10^{-3}$ & $5.2 \times 10^{-1}$ & $9.5 \times 10^{-7}$ & $-6.2 \times 10^{1}$ & 0.29 \\
\hline
\end{tabular}


Table 15 (Continued).

\begin{tabular}{|c|c|c|c|c|c|c|c|c|}
\hline \multirow[b]{2}{*}{ Name } & \multicolumn{8}{|c|}{ Beta values } \\
\hline & $\beta_{0}$ & $\beta_{1}$ & $\beta_{2}$ & $\beta_{3}$ & $\beta_{4}$ & $\beta_{5}$ & $\beta_{6}$ & $R^{2}$ \\
\hline FT9h & $-1.4 \times 10^{-4}$ & $1.6 \times 10^{-1}$ & $-2.1 \times 10^{-3}$ & $-2.5 \times 10^{-3}$ & $8.7 \times 10^{-1}$ & $1.8 \times 10^{-6}$ & $-1.1 \times 10^{2}$ & 0.19 \\
\hline FT7h & $-1.1 \times 10^{-4}$ & $-6.6 \times 10^{-2}$ & $7.4 \times 10^{-5}$ & $-1.4 \times 10^{-3}$ & $-2.4 \times 10^{-2}$ & $-6.4 \times 10^{-8}$ & $1.0 \times 10^{1}$ & 0.16 \\
\hline FC5h & $-7.0 \times 10^{-4}$ & $-8.1 \times 10^{-2}$ & $1.1 \times 10^{-4}$ & $-1.0 \times 10^{-3}$ & $-4.7 \times 10^{-2}$ & $-7.6 \times 10^{-8}$ & $1.4 \times 10^{1}$ & 0.09 \\
\hline FC3h & $4.2 \times 10^{-4}$ & $-1.1 \times 10^{-1}$ & $-6.2 \times 10^{-4}$ & $-1.2 \times 10^{-3}$ & $2.4 \times 10^{-1}$ & $5.3 \times 10^{-7}$ & $-2.4 \times 10^{1}$ & 0.16 \\
\hline FC1h & $1.9 \times 10^{-3}$ & $-1.2 \times 10^{-1}$ & $-1.6 \times 10^{-3}$ & $-1.3 \times 10^{-3}$ & $6.1 \times 10^{-1}$ & $1.3 \times 10^{-6}$ & $-7.1 \times 10^{1}$ & 0.20 \\
\hline FC2h & $-9.3 \times 10^{-5}$ & $-1.7 \times 10^{-1}$ & $-3.9 \times 10^{-3}$ & $-1.4 \times 10^{-3}$ & $1.5 \times 10^{0}$ & $3.3 \times 10^{-6}$ & $-1.9 \times 10^{2}$ & 0.24 \\
\hline $\mathrm{FC} 4 \mathrm{~h}$ & $-1.5 \times 10^{-5}$ & $-1.1 \times 10^{-1}$ & $-3.4 \times 10^{-3}$ & $-1.2 \times 10^{-3}$ & $1.3 \times 10^{0}$ & $2.9 \times 10^{-6}$ & $-1.7 \times 10^{2}$ & 0.24 \\
\hline FC6h & $-1.0 \times 10^{-3}$ & $-9.9 \times 10^{-2}$ & $-1.8 \times 10^{-3}$ & $-1.0 \times 10^{-3}$ & $7.1 \times 10^{-1}$ & $1.5 \times 10^{-6}$ & $-8.8 \times 10^{1}$ & 0.15 \\
\hline FT8h & $-1.4 \times 10^{-3}$ & $-2.2 \times 10^{-2}$ & $-9.6 \times 10^{-4}$ & $-1.1 \times 10^{-3}$ & $4.1 \times 10^{-1}$ & $7.6 \times 10^{-7}$ & $-5.1 \times 10^{1}$ & 0.16 \\
\hline FT10h & $7.7 \times 10^{-3}$ & $1.7 \times 10^{-1}$ & $-4.3 \times 10^{-3}$ & $-2.5 \times 10^{-3}$ & $1.7 \times 10^{0}$ & $3.7 \times 10^{-6}$ & $-2.1 \times 10^{2}$ & 0.24 \\
\hline T9h & $4.2 \times 10^{-3}$ & $1.4 \times 10^{-1}$ & $-1.1 \times 10^{-3}$ & $-2.4 \times 10^{-3}$ & $4.8 \times 10^{-1}$ & $8.6 \times 10^{-7}$ & $-6.1 \times 10^{1}$ & 0.24 \\
\hline $\mathrm{T} 7 \mathrm{~h}$ & $1.4 \times 10^{-4}$ & $-8.8 \times 10^{-2}$ & $4.8 \times 10^{-4}$ & $-1.4 \times 10^{-3}$ & $-1.7 \times 10^{-1}$ & $-4.4 \times 10^{-7}$ & $2.6 \times 10^{1}$ & 0.24 \\
\hline $\mathrm{C} 5 \mathrm{~h}$ & $-1.4 \times 10^{-3}$ & $-5.6 \times 10^{-2}$ & $-3.7 \times 10^{-4}$ & $-1.2 \times 10^{-3}$ & $1.6 \times 10^{-1}$ & $2.8 \times 10^{-7}$ & $-1.5 \times 10^{1}$ & 0.12 \\
\hline C3h & $5.0 \times 10^{-4}$ & $-4.5 \times 10^{-2}$ & $-8.2 \times 10^{-4}$ & $-1.6 \times 10^{-3}$ & $3.3 \times 10^{-1}$ & $6.6 \times 10^{-7}$ & $-3.9 \times 10^{1}$ & 0.24 \\
\hline C1h & $2.3 \times 10^{-3}$ & $-6.4 \times 10^{-2}$ & $-1.5 \times 10^{-3}$ & $-1.5 \times 10^{-3}$ & $6.0 \times 10^{-1}$ & $1.3 \times 10^{-6}$ & $-7.2 \times 10^{1}$ & 0.21 \\
\hline $\mathrm{C} 2 \mathrm{~h}$ & $2.9 \times 10^{-3}$ & $-2.5 \times 10^{-1}$ & $-3.7 \times 10^{-3}$ & $-1.5 \times 10^{-3}$ & $1.4 \times 10^{0}$ & $3.2 \times 10^{-6}$ & $-1.7 \times 10^{2}$ & 0.16 \\
\hline $\mathrm{C} 4 \mathrm{~h}$ & $-1.3 \times 10^{-3}$ & $-9.0 \times 10^{-2}$ & $-2.2 \times 10^{-3}$ & $-1.4 \times 10^{-3}$ & $8.5 \times 10^{-1}$ & $1.8 \times 10^{-6}$ & $-1.0 \times 10^{2}$ & 0.18 \\
\hline C6h & $-1.6 \times 10^{-3}$ & $-6.9 \times 10^{-2}$ & $-7.4 \times 10^{-4}$ & $-1.2 \times 10^{-3}$ & $3.1 \times 10^{-1}$ & $6.1 \times 10^{-7}$ & $-3.5 \times 10^{1}$ & 0.18 \\
\hline T8h & $-2.5 \times 10^{-3}$ & $-9.5 \times 10^{-2}$ & $-1.2 \times 10^{-3}$ & $-1.2 \times 10^{-3}$ & $5.0 \times 10^{-1}$ & $9.9 \times 10^{-7}$ & $-6.1 \times 10^{1}$ & 0.14 \\
\hline T10h & $-3.8 \times 10^{-3}$ & $-1.2 \times 10^{-1}$ & $-4.7 \times 10^{-3}$ & $-2.1 \times 10^{-3}$ & $1.8 \times 10^{0}$ & $4.0 \times 10^{-6}$ & $-2.3 \times 10^{2}$ & 0.19 \\
\hline TP9h & $-5.8 \times 10^{-4}$ & $4.6 \times 10^{-2}$ & $-9.3 \times 10^{-4}$ & $-2.2 \times 10^{-3}$ & $4.1 \times 10^{-1}$ & $6.9 \times 10^{-7}$ & $-5.1 \times 10^{1}$ & 0.20 \\
\hline TP7h & $-1.1 \times 10^{-3}$ & $-9.4 \times 10^{-2}$ & $1.4 \times 10^{-3}$ & $-1.7 \times 10^{-3}$ & $-5.0 \times 10^{-1}$ & $-1.2 \times 10^{-6}$ & $6.7 \times 10^{1}$ & 0.27 \\
\hline CP5h & $-7.7 \times 10^{-4}$ & $-5.2 \times 10^{-2}$ & $1.6 \times 10^{-4}$ & $-1.5 \times 10^{-3}$ & $-3.2 \times 10^{-2}$ & $-2.0 \times 10^{-7}$ & $7.0 \times 10^{0}$ & 0.21 \\
\hline CP3h & $1.3 \times 10^{-4}$ & $-2.4 \times 10^{-2}$ & $-4.4 \times 10^{-5}$ & $-1.7 \times 10^{-3}$ & $4.9 \times 10^{-2}$ & $-3.0 \times 10^{-8}$ & $-3.7 \times 10^{0}$ & 0.30 \\
\hline CP1h & $7.1 \times 10^{-4}$ & $9.5 \times 10^{-3}$ & $-7.4 \times 10^{-4}$ & $-1.7 \times 10^{-3}$ & $3.2 \times 10^{-1}$ & $5.7 \times 10^{-7}$ & $-3.8 \times 10^{1}$ & 0.30 \\
\hline $\mathrm{CP} 2 \mathrm{~h}$ & $2.1 \times 10^{-3}$ & $-7.8 \times 10^{-2}$ & $-1.2 \times 10^{-3}$ & $-1.9 \times 10^{-3}$ & $4.9 \times 10^{-1}$ & $1.0 \times 10^{-6}$ & $-5.7 \times 10^{1}$ & 0.31 \\
\hline CP4h & $-5.2 \times 10^{-5}$ & $-4.5 \times 10^{-2}$ & $-5.6 \times 10^{-4}$ & $-1.7 \times 10^{-3}$ & $2.4 \times 10^{-1}$ & $4.4 \times 10^{-7}$ & $-2.7 \times 10^{1}$ & 0.31 \\
\hline CP6h & $-1.7 \times 10^{-3}$ & $-6.1 \times 10^{-2}$ & $4.4 \times 10^{-4}$ & $-1.5 \times 10^{-3}$ & $-1.6 \times 10^{-1}$ & $-4.0 \times 10^{-7}$ & $2.5 \times 10^{1}$ & 0.22 \\
\hline TP8h & $1.6 \times 10^{-3}$ & $-7.0 \times 10^{-3}$ & $4.7 \times 10^{-4}$ & $-1.6 \times 10^{-3}$ & $-1.7 \times 10^{-1}$ & $-4.4 \times 10^{-7}$ & $2.6 \times 10^{1}$ & 0.23 \\
\hline TP10h & $-8.7 \times 10^{-4}$ & $-1.0 \times 10^{-2}$ & $-1.8 \times 10^{-3}$ & $-2.1 \times 10^{-3}$ & $7.0 \times 10^{-1}$ & $1.5 \times 10^{-6}$ & $-8.4 \times 10^{1}$ & 0.26 \\
\hline P9h & $-9.6 \times 10^{-4}$ & $6.0 \times 10^{-2}$ & $-2.0 \times 10^{-3}$ & $-2.0 \times 10^{-3}$ & $8.1 \times 10^{-1}$ & $1.7 \times 10^{-6}$ & $-1.0 \times 10^{2}$ & 0.39 \\
\hline P7h & $1.2 \times 10^{-3}$ & $-1.1 \times 10^{-1}$ & $1.7 \times 10^{-3}$ & $-2.0 \times 10^{-3}$ & $-6.7 \times 10^{-1}$ & $-1.4 \times 10^{-6}$ & $9.3 \times 10^{1}$ & 0.40 \\
\hline P5h & $1.8 \times 10^{-4}$ & $-6.5 \times 10^{-2}$ & $1.5 \times 10^{-4}$ & $-1.7 \times 10^{-3}$ & $-2.8 \times 10^{-2}$ & $-2.0 \times 10^{-7}$ & $6.1 \times 10^{0}$ & 0.28 \\
\hline
\end{tabular}


Table 15 (Continued).

\begin{tabular}{|c|c|c|c|c|c|c|c|c|}
\hline \multirow[b]{2}{*}{ Name } & \multicolumn{8}{|c|}{ Beta values } \\
\hline & $\beta_{0}$ & $\beta_{1}$ & $\beta_{2}$ & $\beta_{3}$ & $\beta_{4}$ & $\beta_{5}$ & $\beta_{6}$ & $R^{2}$ \\
\hline $\mathrm{P} 3 \mathrm{~h}$ & $6.8 \times 10^{-4}$ & $-5.6 \times 10^{-2}$ & $-1.3 \times 10^{-3}$ & $-1.8 \times 10^{-3}$ & $5.1 \times 10^{-1}$ & $1.0 \times 10^{-6}$ & $-6.2 \times 10^{1}$ & $\overline{0.34}$ \\
\hline $\mathrm{P} 1 \mathrm{~h}$ & $6.5 \times 10^{-4}$ & $-2.4 \times 10^{-2}$ & $-7.8 \times 10^{-4}$ & $-1.9 \times 10^{-3}$ & $3.4 \times 10^{-1}$ & $6.1 \times 10^{-7}$ & $-4.1 \times 10^{1}$ & 0.34 \\
\hline $\mathrm{P} 2 \mathrm{~h}$ & $6.8 \times 10^{-4}$ & $-2.7 \times 10^{-2}$ & $-8.6 \times 10^{-4}$ & $-1.9 \times 10^{-3}$ & $3.7 \times 10^{-1}$ & $6.7 \times 10^{-7}$ & $-4.5 \times 10^{1}$ & 0.33 \\
\hline $\mathrm{P} 4 \mathrm{~h}$ & $7.7 \times 10^{-4}$ & $-5.1 \times 10^{-2}$ & $-1.0 \times 10^{-3}$ & $-1.9 \times 10^{-3}$ & $4.1 \times 10^{-1}$ & $8.2 \times 10^{-7}$ & $-4.9 \times 10^{1}$ & 0.32 \\
\hline P6h & $7.3 \times 10^{-4}$ & $-6.4 \times 10^{-2}$ & $-1.7 \times 10^{-4}$ & $-1.6 \times 10^{-3}$ & $7.7 \times 10^{-2}$ & $1.2 \times 10^{-7}$ & $-4.6 \times 10^{0}$ & 0.27 \\
\hline P8h & $1.8 \times 10^{-3}$ & $-2.2 \times 10^{-2}$ & $1.5 \times 10^{-3}$ & $-1.6 \times 10^{-3}$ & $-5.9 \times 10^{-1}$ & $-1.3 \times 10^{-6}$ & $8.3 \times 10^{1}$ & 0.34 \\
\hline P10h & $-1.9 \times 10^{-4}$ & $-7.9 \times 10^{-2}$ & $-1.6 \times 10^{-3}$ & $-2.1 \times 10^{-3}$ & $6.7 \times 10^{-1}$ & $1.3 \times 10^{-6}$ & $-8.3 \times 10^{1}$ & 0.28 \\
\hline PO9h & $6.0 \times 10^{-4}$ & $9.1 \times 10^{-3}$ & $-3.0 \times 10^{-3}$ & $-2.1 \times 10^{-3}$ & $1.2 \times 10^{0}$ & $2.5 \times 10^{-6}$ & $-1.5 \times 10^{2}$ & 0.32 \\
\hline PO7h & $-11.3 \times 10^{-3}$ & $-5.0 \times 10^{-3}$ & $3.7 \times 10^{-4}$ & $-2.1 \times 10^{-3}$ & $-1.4 \times 10^{-1}$ & $-3.1 \times 10^{-7}$ & $2.8 \times 10^{1}$ & 0.39 \\
\hline PO5h & $-3.5 \times 10^{-4}$ & $-8.4 \times 10^{-2}$ & $-3.4 \times 10^{-4}$ & $-1.8 \times 10^{-3}$ & $1.5 \times 10^{-1}$ & $2.6 \times 10^{-7}$ & $-1.5 \times 10^{1}$ & 0.36 \\
\hline PO3h & $8.5 \times 10^{-4}$ & $-6.5 \times 10^{-2}$ & $-1.6 \times 10^{-3}$ & $-1.8 \times 10^{-3}$ & $6.5 \times 10^{-1}$ & $1.4 \times 10^{-6}$ & $-7.9 \times 10^{1}$ & 0.31 \\
\hline P01h & $8.5 \times 10^{-4}$ & $-3.9 \times 10^{-2}$ & $-1.7 \times 10^{-3}$ & $-1.8 \times 10^{-3}$ & $6.9 \times 10^{-1}$ & $1.4 \times 10^{-6}$ & $-8.4 \times 10^{1}$ & 0.37 \\
\hline $\mathrm{PO} 2 \mathrm{~h}$ & $1.0 \times 10^{-3}$ & $-4.0 \times 10^{-2}$ & $-9.1 \times 10^{-4}$ & $-1.8 \times 10^{-3}$ & $3.8 \times 10^{-1}$ & $7.3 \times 10^{-7}$ & $-4.5 \times 10^{1}$ & 0.36 \\
\hline $\mathrm{PO} 4 \mathrm{~h}$ & $6.6 \times 10^{-4}$ & $-7.8 \times 10^{-2}$ & $-1.4 \times 10^{-3}$ & $-1.8 \times 10^{-3}$ & $5.8 \times 10^{-1}$ & $1.2 \times 10^{-6}$ & $-7.1 \times 10^{1}$ & 0.35 \\
\hline PO6h & $2.3 \times 10^{-4}$ & $-9.2 \times 10^{-2}$ & $-4.9 \times 10^{-4}$ & $-1.8 \times 10^{-3}$ & $2.0 \times 10^{-1}$ & $4.0 \times 10^{-7}$ & $-2.1 \times 10^{1}$ & 0.39 \\
\hline PO8h & $7.8 \times 10^{-4}$ & $-8.9 \times 10^{-2}$ & $1.4 \times 10^{-3}$ & $-2.1 \times 10^{-3}$ & $-5.6 \times 10^{-1}$ & $-1.2 \times 10^{-6}$ & $8.0 \times 10^{1}$ & 0.55 \\
\hline P010h & $7.2 \times 10^{-4}$ & $-7.3 \times 10^{-2}$ & $-3.2 \times 10^{-3}$ & $-2.2 \times 10^{-3}$ & $1.3 \times 10^{0}$ & $2.8 \times 10^{-6}$ & $-1.6 \times 10^{2}$ & 0.36 \\
\hline O1h & $-9.1 \times 10^{-4}$ & $-1.0 \times 10^{-1}$ & $4.7 \times 10^{-5}$ & $-2.2 \times 10^{-3}$ & $-2.0 \times 10^{-2}$ & $-2.6 \times 10^{-8}$ & $1.0 \times 10^{1}$ & 0.39 \\
\hline $\mathrm{O} 2 \mathrm{~h}$ & $2.4 \times 10^{-3}$ & $2.7 \times 10^{-2}$ & $7.5 \times 10^{-4}$ & $-2.1 \times 10^{-3}$ & $-2.9 \times 10^{-1}$ & $-6.3 \times 10^{-7}$ & $4.4 \times 10^{1}$ & 0.35 \\
\hline I1h & $1.6 \times 10^{-3}$ & $-8.1 \times 10^{-2}$ & $-3.6 \times 10^{-3}$ & $-2.0 \times 10^{-3}$ & $1.4 \times 10^{0}$ & $3.0 \times 10^{-6}$ & $-1.8 \times 10^{2}$ & 0.86 \\
\hline $\mathrm{I} 2 \mathrm{~h}$ & $8.2 \times 10^{-4}$ & $-8.9 \times 10^{-2}$ & $-2.2 \times 10^{-3}$ & $-1.9 \times 10^{-3}$ & $9.0 \times 10^{-1}$ & $1.9 \times 10^{-6}$ & $-1.1 \times 10^{2}$ & 0.48 \\
\hline AFp9 & $4.2 \times 10^{-3}$ & $-5.6 \times 10^{-2}$ & $-3.6 \times 10^{-3}$ & $-2.3 \times 10^{-3}$ & $1.4 \times 10^{0}$ & $3.1 \times 10^{-6}$ & $-1.7 \times 10^{2}$ & 0.48 \\
\hline AFp7 & $5.1 \times 10^{-3}$ & $-1.4 \times 10^{-1}$ & $-2.1 \times 10^{-3}$ & $-2.3 \times 10^{-3}$ & $7.8 \times 10^{-1}$ & $1.8 \times 10^{-6}$ & $-1.0 \times 10^{2}$ & 0.19 \\
\hline AFp5 & $2.5 \times 10^{-4}$ & $-8.4 \times 10^{-2}$ & $1.1 \times 10^{-4}$ & $-1.2 \times 10^{-3}$ & $-1.8 \times 10^{-2}$ & $-1.4 \times 10^{-7}$ & $5.5 \times 10^{0}$ & 0.19 \\
\hline AFp3 & $5.8 \times 10^{-4}$ & $-8.4 \times 10^{-2}$ & $3.7 \times 10^{-4}$ & $-1.1 \times 10^{-3}$ & $-1.3 \times 10^{-1}$ & $-3.5 \times 10^{-7}$ & $2.0 \times 10^{1}$ & 0.18 \\
\hline AFp1 & $1.9 \times 10^{-3}$ & $-1.2 \times 10^{-1}$ & $2.5 \times 10^{-4}$ & $-1.3 \times 10^{-3}$ & $-9.8 \times 10^{-2}$ & $-2.2 \times 10^{-7}$ & $1.9 \times 10^{1}$ & 0.16 \\
\hline AFpz & $1.8 \times 10^{-3}$ & $-1.1 \times 10^{-1}$ & $-9.7 \times 10^{-5}$ & $-1.5 \times 10^{-3}$ & $3.8 \times 10^{-2}$ & $8.4 \times 10^{-8}$ & $1.7 \times 10^{0}$ & 0.18 \\
\hline AFp2 & $1.4 \times 10^{-3}$ & $-1.1 \times 10^{-1}$ & $2.1 \times 10^{-4}$ & $-1.4 \times 10^{-3}$ & $-8.6 \times 10^{-2}$ & $-1.8 \times 10^{-7}$ & $1.8 \times 10^{1}$ & 0.18 \\
\hline AFp4 & $3.8 \times 10^{-4}$ & $-8.1 \times 10^{-2}$ & $4.3 \times 10^{-4}$ & $-1.4 \times 10^{-3}$ & $-1.6 \times 10^{-1}$ & $-3.9 \times 10^{-7}$ & $2.5 \times 10^{1}$ & 0.20 \\
\hline AFp6 & $6.8 \times 10^{-4}$ & $-6.8 \times 10^{-2}$ & $5.5 \times 10^{-4}$ & $-1.4 \times 10^{-3}$ & $-2.2 \times 10^{-1}$ & $-4.7 \times 10^{-7}$ & $3.5 \times 10^{1}$ & 0.21 \\
\hline AFp8 & $2.5 \times 10^{-3}$ & $1.4 \times 10^{-2}$ & $-4.2 \times 10^{-4}$ & $-2.3 \times 10^{-3}$ & $1.8 \times 10^{-1}$ & $3.3 \times 10^{-7}$ & $-1.6 \times 10^{1}$ & 0.17 \\
\hline AFp10 & $3.4 \times 10^{-3}$ & $-5.0 \times 10^{-2}$ & $-2.2 \times 10^{-3}$ & $-2.3 \times 10^{-3}$ & $8.8 \times 10^{-1}$ & $1.8 \times 10^{-6}$ & $-1.1 \times 10^{2}$ & 0.36 \\
\hline AFF9 & $3.9 \times 10^{-3}$ & $-1.8 \times 10^{-2}$ & $-3.5 \times 10^{-3}$ & $-2.6 \times 10^{-3}$ & $1.4 \times 10^{0}$ & $2.9 \times 10^{-6}$ & $-1.8 \times 10^{2}$ & 0.73 \\
\hline
\end{tabular}


Table 15 (Continued).

\begin{tabular}{|c|c|c|c|c|c|c|c|c|}
\hline \multirow[b]{2}{*}{ Name } & \multicolumn{8}{|c|}{ Beta values } \\
\hline & $\beta_{0}$ & $\beta_{1}$ & $\beta_{2}$ & $\beta_{3}$ & $\beta_{4}$ & $\beta_{5}$ & $\beta_{6}$ & $R^{2}$ \\
\hline$\overline{\mathrm{AFF} 7}$ & $-7.8 \times 10^{-4}$ & $-6.5 \times 10^{-2}$ & $-2.8 \times 10^{-3}$ & $-1.6 \times 10^{-3}$ & $1.1 \times 10^{0}$ & $2.3 \times 10^{-6}$ & $-1.4 \times 10^{2}$ & 0.20 \\
\hline AFF5 & $8.3 \times 10^{-4}$ & $-1.0 \times 10^{-1}$ & $-6.9 \times 10^{-5}$ & $-8.4 \times 10^{-4}$ & $4.4 \times 10^{-2}$ & $3.0 \times 10^{-8}$ & $-8.4 \times 10^{-1}$ & 0.18 \\
\hline AFF3 & $8.1 \times 10^{-4}$ & $-1.0 \times 10^{-1}$ & $-1.4 \times 10^{-4}$ & $-1.1 \times 10^{-3}$ & $6.4 \times 10^{-2}$ & $1.0 \times 10^{-7}$ & $-2.7 \times 10^{0}$ & 0.15 \\
\hline AFF1 & $6.4 \times 10^{-4}$ & $-1.1 \times 10^{-1}$ & $-6.6 \times 10^{-5}$ & $-1.1 \times 10^{-3}$ & $2.5 \times 10^{-2}$ & $5.6 \times 10^{-8}$ & $4.0 \times 10^{0}$ & 0.15 \\
\hline $\mathrm{AFFz}$ & $1.5 \times 10^{-3}$ & $-1.4 \times 10^{-1}$ & $-1.0 \times 10^{-3}$ & $-1.3 \times 10^{-3}$ & $4.1 \times 10^{-1}$ & $8.7 \times 10^{-7}$ & $-4.6 \times 10^{1}$ & 0.18 \\
\hline AFF2 & $1.2 \times 10^{-3}$ & $-1.3 \times 10^{-1}$ & $-9.1 \times 10^{-4}$ & $-1.2 \times 10^{-3}$ & $3.7 \times 10^{-1}$ & $7.4 \times 10^{-7}$ & $-4.3 \times 10^{1}$ & 0.19 \\
\hline AFF4 & $3.0 \times 10^{-4}$ & $-1.1 \times 10^{-1}$ & $-9.0 \times 10^{-4}$ & $-1.1 \times 10^{-3}$ & $3.6 \times 10^{-1}$ & $7.5 \times 10^{-7}$ & $-4.1 \times 10^{1}$ & 0.18 \\
\hline AFF6 & $-1.3 \times 10^{-3}$ & $-1.5 \times 10^{-1}$ & $-1.3 \times 10^{-3}$ & $-9.9 \times 10^{-4}$ & $4.9 \times 10^{-1}$ & $1.1 \times 10^{-6}$ & $-5.7 \times 10^{1}$ & 0.11 \\
\hline AFF8 & $6.2 \times 10^{-4}$ & $-1.7 \times 10^{-1}$ & $-8.4 \times 10^{-4}$ & $-1.5 \times 10^{-3}$ & $3.4 \times 10^{-1}$ & $7.1 \times 10^{-7}$ & $-3.8 \times 10^{1}$ & 0.24 \\
\hline AFF10 & $2.8 \times 10^{-3}$ & $-6.9 \times 10^{-2}$ & $-3.3 \times 10^{-3}$ & $-2.8 \times 10^{-3}$ & $1.3 \times 10^{0}$ & $2.8 \times 10^{-6}$ & $-1.6 \times 10^{2}$ & 0.70 \\
\hline FFT9 & $1.8 \times 10^{-3}$ & $2.1 \times 10^{-3}$ & $-1.5 \times 10^{-3}$ & $-2.7 \times 10^{-3}$ & $5.9 \times 10^{-1}$ & $1.2 \times 10^{-6}$ & $-7.3 \times 10^{1}$ & 0.83 \\
\hline FFT7 & $-1.4 \times 10^{-3}$ & $3.0 \times 10^{-2}$ & $1.7 \times 10^{-3}$ & $-1.7 \times 10^{-3}$ & $-6.3 \times 10^{-1}$ & $-1.5 \times 10^{-6}$ & $8.4 \times 10^{1}$ & 0.33 \\
\hline FFC5 & $-1.4 \times 10^{-3}$ & $-3.9 \times 10^{-2}$ & $2.1 \times 10^{-4}$ & $-9.3 \times 10^{-4}$ & $-8.3 \times 10^{-2}$ & $-1.7 \times 10^{-7}$ & $1.7 \times 10^{1}$ & 0.07 \\
\hline FFC3 & $3.5 \times 10^{-4}$ & $-1.1 \times 10^{-1}$ & $-6.7 \times 10^{-4}$ & $-1.1 \times 10^{-3}$ & $2.6 \times 10^{-1}$ & $5.8 \times 10^{-7}$ & $-2.5 \times 10^{1}$ & 0.11 \\
\hline FFC1 & $1.5 \times 10^{-3}$ & $-1.2 \times 10^{-1}$ & $-1.0 \times 10^{-3}$ & $-1.1 \times 10^{-3}$ & $3.8 \times 10^{-1}$ & $9.1 \times 10^{-7}$ & $-3.9 \times 10^{1}$ & 0.14 \\
\hline $\mathrm{FFCz}$ & $7.7 \times 10^{-4}$ & $-1.1 \times 10^{-1}$ & $-1.8 \times 10^{-3}$ & $-1.2 \times 10^{-3}$ & $6.9 \times 10^{-1}$ & $1.6 \times 10^{-6}$ & $-8.2 \times 10^{1}$ & 0.21 \\
\hline FFC2 & $9.5 \times 10^{-4}$ & $-1.0 \times 10^{-1}$ & $-3.0 \times 10^{-3}$ & $-1.2 \times 10^{-3}$ & $1.2 \times 10^{0}$ & $2.5 \times 10^{-6}$ & $-1.5 \times 10^{2}$ & 0.25 \\
\hline FFC4 & $4.2 \times 10^{-4}$ & $-7.6 \times 10^{-2}$ & $-1.2 \times 10^{-3}$ & $-1.0 \times 10^{-3}$ & $5.1 \times 10^{-1}$ & $1.0 \times 10^{-6}$ & $-6.2 \times 10^{1}$ & 0.18 \\
\hline FFC6 & $-1.2 \times 10^{-3}$ & $-8.4 \times 10^{-2}$ & $-6.3 \times 10^{-4}$ & $-9.4 \times 10^{-4}$ & $2.7 \times 10^{-1}$ & $5.1 \times 10^{-7}$ & $-3.0 \times 10^{1}$ & 0.14 \\
\hline FFT8 & $2.3 \times 10^{-3}$ & $7.7 \times 10^{-2}$ & $-1.7 \times 10^{-3}$ & $-1.7 \times 10^{-3}$ & $6.8 \times 10^{-1}$ & $1.4 \times 10^{-6}$ & $-8.6 \times 10^{1}$ & 0.11 \\
\hline FFT10 & $1.9 \times 10^{-3}$ & $1.7 \times 10^{-2}$ & $-4.2 \times 10^{-3}$ & $-2.7 \times 10^{-3}$ & $1.7 \times 10^{0}$ & $3.5 \times 10^{-6}$ & $-2.1 \times 10^{2}$ & 0.93 \\
\hline FTT9 & $-2.6 \times 10^{-4}$ & $3.2 \times 10^{-2}$ & $-1.5 \times 10^{-3}$ & $-2.1 \times 10^{-3}$ & $6.1 \times 10^{-1}$ & $1.3 \times 10^{-6}$ & $-7.2 \times 10^{1}$ & 0.71 \\
\hline FTT7 & $1.3 \times 10^{-3}$ & $-8.3 \times 10^{-2}$ & $-1.5 \times 10^{-3}$ & $-2.2 \times 10^{-3}$ & $6.0 \times 10^{-1}$ & $1.3 \times 10^{-6}$ & $-7.1 \times 10^{1}$ & 0.16 \\
\hline FCC5 & $-5.4 \times 10^{-4}$ & $-5.3 \times 10^{-2}$ & $5.2 \times 10^{-4}$ & $-1.1 \times 10^{-3}$ & $-2.1 \times 10^{-1}$ & $-4.4 \times 10^{-7}$ & $3.4 \times 10^{1}$ & 0.09 \\
\hline FCC3 & $-9.8 \times 10^{-4}$ & $-7.1 \times 10^{-2}$ & $-4.0 \times 10^{-5}$ & $-1.2 \times 10^{-3}$ & $2.6 \times 10^{-2}$ & $2.0 \times 10^{-8}$ & $2.4 \times 10^{0}$ & 0.14 \\
\hline $\mathrm{FCC} 1$ & $1.7 \times 10^{-3}$ & $-8.5 \times 10^{-2}$ & $-1.3 \times 10^{-3}$ & $-1.4 \times 10^{-3}$ & $5.3 \times 10^{-1}$ & $1.1 \times 10^{-6}$ & $-6.4 \times 10^{1}$ & 0.21 \\
\hline $\mathrm{FCC} z$ & $1.2 \times 10^{-3}$ & $-2.3 \times 10^{-1}$ & $-3.4 \times 10^{-3}$ & $-1.5 \times 10^{-3}$ & $1.3 \times 10^{0}$ & $2.9 \times 10^{-6}$ & $-1.6 \times 10^{2}$ & 0.20 \\
\hline $\mathrm{FCC} 2$ & $-4.9 \times 10^{-4}$ & $-1.4 \times 10^{-1}$ & $-4.3 \times 10^{-3}$ & $-1.4 \times 10^{-3}$ & $1.7 \times 10^{0}$ & $3.7 \times 10^{-6}$ & $-2.1 \times 10^{2}$ & 0.19 \\
\hline FCC4 & $-1.1 \times 10^{-3}$ & $-9.2 \times 10^{-2}$ & $-2.1 \times 10^{-3}$ & $-1.2 \times 10^{-3}$ & $8.5 \times 10^{-1}$ & $1.8 \times 10^{-6}$ & $-1.0 \times 10^{2}$ & 0.13 \\
\hline FCC6 & $-1.9 \times 10^{-3}$ & $-7.4 \times 10^{-2}$ & $-1.3 \times 10^{-3}$ & $-1.0 \times 10^{-3}$ & $5.3 \times 10^{-1}$ & $1.1 \times 10^{-6}$ & $-6.5 \times 10^{1}$ & 0.12 \\
\hline FTT8 & $-3.1 \times 10^{-3}$ & $-1.7 \times 10^{-2}$ & $-1.5 \times 10^{-3}$ & $-1.5 \times 10^{-3}$ & $6.1 \times 10^{-1}$ & $1.2 \times 10^{-6}$ & $-7.8 \times 10^{1}$ & 0.14 \\
\hline FTT10 & $3.4 \times 10^{-3}$ & $6.2 \times 10^{-2}$ & $-3.2 \times 10^{-3}$ & $-1.9 \times 10^{-3}$ & $1.3 \times 10^{0}$ & $2.6 \times 10^{-6}$ & $-1.7 \times 10^{2}$ & 0.33 \\
\hline TTP9 & $1.7 \times 10^{-3}$ & $-5.1 \times 10^{-2}$ & $-1.1 \times 10^{-3}$ & $-1.9 \times 10^{-3}$ & $4.0 \times 10^{-1}$ & $9.4 \times 10^{-7}$ & $-4.4 \times 10^{1}$ & 0.42 \\
\hline
\end{tabular}


Table 15 (Continued).

\begin{tabular}{|c|c|c|c|c|c|c|c|c|}
\hline \multirow[b]{2}{*}{ Name } & \multicolumn{8}{|c|}{ Beta values } \\
\hline & $\beta_{0}$ & $\beta_{1}$ & $\beta_{2}$ & $\beta_{3}$ & $\beta_{4}$ & $\beta_{5}$ & $\beta_{6}$ & $R^{2}$ \\
\hline TTP7 & $-1.4 \times 10^{-3}$ & $-4.0 \times 10^{-2}$ & $1.2 \times 10^{-3}$ & $-2.2 \times 10^{-3}$ & $-4.3 \times 10^{-1}$ & $-1.1 \times 10^{-6}$ & $5.9 \times 10^{1}$ & $\overline{0.28}$ \\
\hline CCP5 & $-7.9 \times 10^{-4}$ & $-7.9 \times 10^{-2}$ & $3.5 \times 10^{-4}$ & $-1.3 \times 10^{-3}$ & $-1.1 \times 10^{-1}$ & $-3.3 \times 10^{-7}$ & $1.9 \times 10^{1}$ & 0.18 \\
\hline $\mathrm{CCP} 3$ & $-4.5 \times 10^{-4}$ & $-3.5 \times 10^{-2}$ & $-3.8 \times 10^{-4}$ & $-1.5 \times 10^{-3}$ & $1.7 \times 10^{-1}$ & $2.8 \times 10^{-7}$ & $-1.8 \times 10^{1}$ & 0.24 \\
\hline CCP1 & $6.9 \times 10^{-4}$ & $9.1 \times 10^{-3}$ & $-7.7 \times 10^{-4}$ & $-1.6 \times 10^{-3}$ & $3.2 \times 10^{-1}$ & $6.1 \times 10^{-7}$ & $-3.8 \times 10^{1}$ & 0.25 \\
\hline $\mathrm{CCPz}$ & $2.3 \times 10^{-3}$ & $-8.2 \times 10^{-2}$ & $-1.4 \times 10^{-3}$ & $-1.7 \times 10^{-3}$ & $5.4 \times 10^{-1}$ & $1.2 \times 10^{-6}$ & $-6.2 \times 10^{1}$ & 0.24 \\
\hline CCP2 & $2.3 \times 10^{-3}$ & $-1.3 \times 10^{-1}$ & $-2.0 \times 10^{-3}$ & $-1.6 \times 10^{-3}$ & $7.7 \times 10^{-1}$ & $1.7 \times 10^{-6}$ & $-9.1 \times 10^{1}$ & 0.21 \\
\hline $\mathrm{CCP} 4$ & $-1.5 \times 10^{-3}$ & $-6.6 \times 10^{-2}$ & $-6.3 \times 10^{-4}$ & $-1.4 \times 10^{-3}$ & $2.6 \times 10^{-1}$ & $5.1 \times 10^{-7}$ & $-2.9 \times 10^{1}$ & 0.23 \\
\hline CCP6 & $-1.5 \times 10^{-3}$ & $-3.9 \times 10^{-2}$ & $-1.0 \times 10^{-5}$ & $-1.2 \times 10^{-3}$ & $2.8 \times 10^{-2}$ & $-2.2 \times 10^{-8}$ & $1.8 \times 10^{-1}$ & 0.16 \\
\hline TTP8 & $-2.8 \times 10^{-4}$ & $-1.1 \times 10^{-1}$ & $5.0 \times 10^{-4}$ & $-2.0 \times 10^{-3}$ & $-1.7 \times 10^{-1}$ & $-4.6 \times 10^{-7}$ & $2.6 \times 10^{1}$ & 0.19 \\
\hline TTP10 & $2.1 \times 10^{-3}$ & $-7.2 \times 10^{-2}$ & $-1.8 \times 10^{-3}$ & $-1.7 \times 10^{-3}$ & $7.3 \times 10^{-1}$ & $1.5 \times 10^{-6}$ & $-9.0 \times 10^{1}$ & 0.13 \\
\hline TPP9 & $-1.0 \times 10^{-3}$ & $-1.5 \times 10^{-2}$ & $-9.9 \times 10^{-4}$ & $-1.6 \times 10^{-3}$ & $4.1 \times 10^{-1}$ & $7.8 \times 10^{-7}$ & $-5.1 \times 10^{1}$ & 0.78 \\
\hline TPP7 & $-8.6 \times 10^{-5}$ & $-4.0 \times 10^{-2}$ & $-9.1 \times 10^{-5}$ & $-2.0 \times 10^{-3}$ & $6.2 \times 10^{-2}$ & $5.9 \times 10^{-8}$ & $-3.0 \times 10^{0}$ & 0.29 \\
\hline CPP5 & $3.3 \times 10^{-4}$ & $-9.8 \times 10^{-2}$ & $5.8 \times 10^{-4}$ & $-1.6 \times 10^{-3}$ & $-2.0 \times 10^{-1}$ & $-5.4 \times 10^{-7}$ & $3.0 \times 10^{1}$ & 0.31 \\
\hline CPP3 & $-4.5 \times 10^{-5}$ & $-5.4 \times 10^{-2}$ & $7.0 \times 10^{-7}$ & $-1.7 \times 10^{-3}$ & $3.2 \times 10^{-2}$ & $-66.8 \times 10^{-8}$ & $-1.6 \times 10^{0}$ & 0.31 \\
\hline CPP1 & $8.0 \times 10^{-4}$ & $-1.9 \times 10^{-2}$ & $-8.1 \times 10^{-4}$ & $-1.8 \times 10^{-3}$ & $3.4 \times 10^{-1}$ & $6.3 \times 10^{-7}$ & $-4.2 \times 10^{1}$ & 0.34 \\
\hline $\mathrm{CPPz}$ & $5.5 \times 10^{-4}$ & $-1.4 \times 10^{-2}$ & $-6.2 \times 10^{-4}$ & $-1.9 \times 10^{-3}$ & $2.7 \times 10^{-1}$ & $4.6 \times 10^{-7}$ & $-3.2 \times 10^{1}$ & 0.33 \\
\hline CPP2 & $7.3 \times 10^{-4}$ & $-3.8 \times 10^{-2}$ & $-1.2 \times 10^{-3}$ & $-1.9 \times 10^{-3}$ & $4.8 \times 10^{-1}$ & $9.6 \times 10^{-7}$ & $-5.9 \times 10^{1}$ & 0.33 \\
\hline CPP4 & $-8.1 \times 10^{-5}$ & $-5.7 \times 10^{-2}$ & $1.7 \times 10^{-4}$ & $-1.7 \times 10^{-3}$ & $-4.9 \times 10^{-2}$ & $-1.8 \times 10^{-7}$ & $1.1 \times 10^{1}$ & 0.29 \\
\hline CPP6 & $-9.7 \times 10^{-4}$ & $-7.5 \times 10^{-2}$ & $1.1 \times 10^{-3}$ & $-1.5 \times 10^{-3}$ & $-4.2 \times 10^{-1}$ & $-9.5 \times 10^{-7}$ & $6.0 \times 10^{1}$ & 0.21 \\
\hline TPP8 & $4.0 \times 10^{-4}$ & $-2.8 \times 10^{-3}$ & $7.2 \times 10^{-4}$ & $-2.0 \times 10^{-3}$ & $-2.7 \times 10^{-1}$ & $-6.3 \times 10^{-7}$ & $4.0 \times 10^{1}$ & 0.27 \\
\hline TPP10 & $-1.1 \times 10^{-4}$ & $1.8 \times 10^{-1}$ & $5.3 \times 10^{-4}$ & $-1.3 \times 10^{-3}$ & $-1.5 \times 10^{-1}$ & $-5.9 \times 10^{-7}$ & $1.9 \times 10^{1}$ & 0.83 \\
\hline PPO9 & $8.4 \times 10^{-4}$ & $-5.9 \times 10^{-2}$ & $-1.2 \times 10^{-3}$ & $-1.7 \times 10^{-3}$ & $4.8 \times 10^{-1}$ & $9.3 \times 10^{-7}$ & $-6.0 \times 10^{1}$ & 0.80 \\
\hline PPO7 & $4.0 \times 10^{-4}$ & $-2.8 \times 10^{-2}$ & $-1.4 \times 10^{-3}$ & $-2.4 \times 10^{-3}$ & $5.5 \times 10^{-1}$ & $1.2 \times 10^{-6}$ & $-6.2 \times 10^{1}$ & 0.44 \\
\hline PPO5 & $-2.5 \times 10^{-4}$ & $-5.8 \times 10^{-2}$ & $1.2 \times 10^{-4}$ & $-1.8 \times 10^{-3}$ & $-1.9 \times 10^{-2}$ & $-1.5 \times 10^{-7}$ & $5.7 \times 10^{0}$ & 0.31 \\
\hline PPO3 & $2.8 \times 10^{-4}$ & $-7.9 \times 10^{-2}$ & $-1.1 \times 10^{-3}$ & $-1.7 \times 10^{-3}$ & $4.6 \times 10^{-1}$ & $9.1 \times 10^{-7}$ & $-5.5 \times 10^{1}$ & 0.29 \\
\hline PPO1 & $9.0 \times 10^{-4}$ & $-4.3 \times 10^{-2}$ & $-1.9 \times 10^{-3}$ & $-1.8 \times 10^{-3}$ & $7.6 \times 10^{-1}$ & $1.6 \times 10^{-6}$ & $-9.4 \times 10^{1}$ & 0.33 \\
\hline $\mathrm{PPOz}$ & $5.5 \times 10^{-4}$ & $-2.3 \times 10^{-2}$ & $-1.3 \times 10^{-3}$ & $-1.9 \times 10^{-3}$ & $5.2 \times 10^{-1}$ & $1.0 \times 10^{-6}$ & $-6.5 \times 10^{1}$ & 0.33 \\
\hline PPO2 & $8.2 \times 10^{-4}$ & $-5.7 \times 10^{-2}$ & $-1.1 \times 10^{-3}$ & $-1.9 \times 10^{-3}$ & $4.7 \times 10^{-1}$ & $9.1 \times 10^{-7}$ & $-5.7 \times 10^{1}$ & 0.33 \\
\hline PPO4 & $6.6 \times 10^{-4}$ & $-6.8 \times 10^{-2}$ & $-7.9 \times 10^{-4}$ & $-1.8 \times 10^{-3}$ & $3.3 \times 10^{-1}$ & $6.4 \times 10^{-7}$ & $-3.7 \times 10^{1}$ & 0.31 \\
\hline PPO6 & $1.1 \times 10^{-3}$ & $-8.9 \times 10^{-2}$ & $8.0 \times 10^{-5}$ & $-1.7 \times 10^{-3}$ & $-2.4 \times 10^{-2}$ & $-8.2 \times 10^{-8}$ & $8.9 \times 10^{0}$ & 0.36 \\
\hline PPO8 & $-8.0 \times 10^{-4}$ & $-8.5 \times 10^{-3}$ & $3.9 \times 10^{-4}$ & $-2.2 \times 10^{-3}$ & $-1.7 \times 10^{-1}$ & $-2.9 \times 10^{-7}$ & $3.1 \times 10^{1}$ & 0.36 \\
\hline PPO10 & $6.6 \times 10^{-4}$ & $-3.8 \times 10^{-2}$ & $8.0 \times 10^{-4}$ & $-1.9 \times 10^{-3}$ & $-2.9 \times 10^{-1}$ & $-7.8 \times 10^{-7}$ & $4.1 \times 10^{1}$ & 0.47 \\
\hline POO9 & $2.1 \times 10^{-3}$ & $-5.8 \times 10^{-2}$ & $-3.4 \times 10^{-3}$ & $-1.9 \times 10^{-3}$ & $1.3 \times 10^{0}$ & $2.8 \times 10^{-6}$ & $-1.7 \times 10^{2}$ & 0.93 \\
\hline
\end{tabular}


Table 15 (Continued).

\begin{tabular}{|c|c|c|c|c|c|c|c|c|}
\hline \multirow[b]{2}{*}{ Name } & \multicolumn{8}{|c|}{ Beta values } \\
\hline & $\beta_{0}$ & $\beta_{1}$ & $\beta_{2}$ & $\beta_{3}$ & $\beta_{4}$ & $\beta_{5}$ & $\beta_{6}$ & $R^{2}$ \\
\hline POO7 & $-2.0 \times 10^{-4}$ & $1.1 \times 10^{-2}$ & $-4.7 \times 10^{-4}$ & $-2.3 \times 10^{-3}$ & $1.8 \times 10^{-1}$ & $4.0 \times 10^{-7}$ & $-1.4 \times 10^{1}$ & 0.40 \\
\hline POO5 & $4.9 \times 10^{-4}$ & $-4.5 \times 10^{-2}$ & $6.2 \times 10^{-4}$ & $-2.0 \times 10^{-3}$ & $-2.5 \times 10^{-1}$ & $-5.3 \times 10^{-7}$ & $3.8 \times 10^{1}$ & 0.35 \\
\hline POO3 & $-3.1 \times 10^{-4}$ & $-7.4 \times 10^{-2}$ & $-7.4 \times 10^{-4}$ & $-1.9 \times 10^{-3}$ & $3.0 \times 10^{-1}$ & $6.2 \times 10^{-7}$ & $-3.3 \times 10^{1}$ & 0.40 \\
\hline POO1 & $5.4 \times 10^{-4}$ & $-9.4 \times 10^{-2}$ & $-1.1 \times 10^{-3}$ & $-1.9 \times 10^{-3}$ & $4.5 \times 10^{-1}$ & $9.3 \times 10^{-7}$ & $-5.2 \times 10^{1}$ & 0.37 \\
\hline $\mathrm{POOz}$ & $1.1 \times 10^{-3}$ & $-6.2 \times 10^{-2}$ & $-1.0 \times 10^{-3}$ & $-1.8 \times 10^{-3}$ & $4.2 \times 10^{-1}$ & $8.6 \times 10^{-7}$ & $-4.9 \times 10^{1}$ & 0.46 \\
\hline POO2 & $1.4 \times 10^{-3}$ & $-7.8 \times 10^{-2}$ & $-1.0 \times 10^{-3}$ & $-1.8 \times 10^{-3}$ & $4.2 \times 10^{-1}$ & $8.3 \times 10^{-7}$ & $-5.0 \times 10^{1}$ & 0.37 \\
\hline POO4 & $7.5 \times 10^{-4}$ & $-9.2 \times 10^{-2}$ & $-3.0 \times 10^{-4}$ & $-1.8 \times 10^{-3}$ & $1.3 \times 10^{-1}$ & $2.2 \times 10^{-7}$ & $-1.3 \times 10^{1}$ & 0.37 \\
\hline POO6 & $-1.1 \times 10^{-3}$ & $-6.7 \times 10^{-2}$ & $2.9 \times 10^{-4}$ & $-1.9 \times 10^{-3}$ & $-1.3 \times 10^{-1}$ & $-2.0 \times 10^{-7}$ & $2.6 \times 10^{1}$ & 0.46 \\
\hline POO8 & $-1.2 \times 10^{-3}$ & $-1.9 \times 10^{-2}$ & $-1.5 \times 10^{-3}$ & $-2.4 \times 10^{-3}$ & $5.5 \times 10^{-1}$ & $1.3 \times 10^{-6}$ & $-6.1 \times 10^{1}$ & 0.33 \\
\hline P0010 & $1.5 \times 10^{-3}$ & $-6.7 \times 10^{-2}$ & $-2.6 \times 10^{-3}$ & $-2.1 \times 10^{-3}$ & $1.0 \times 10^{0}$ & $2.2 \times 10^{-6}$ & $-1.3 \times 10^{2}$ & 0.78 \\
\hline Ol1 & $-3.8 \times 10^{-4}$ & $-2.1 \times 10^{-2}$ & $-2.3 \times 10^{-3}$ & $-2.2 \times 10^{-3}$ & $9.2 \times 10^{-1}$ & $1.9 \times 10^{-6}$ & $-1.2 \times 10^{2}$ & 0.39 \\
\hline $\mathrm{Olz}$ & $1.3 \times 10^{-4}$ & $-8.5 \times 10^{-2}$ & $-4.0 \times 10^{-3}$ & $-2.1 \times 10^{-3}$ & $1.5 \times 10^{0}$ & $3.4 \times 10^{-6}$ & $-1.9 \times 10^{2}$ & 0.39 \\
\hline $\mathrm{Ol} 2$ & $3.4 \times 10^{-4}$ & $-2.8 \times 10^{-2}$ & $-4.1 \times 10^{-3}$ & $-2.2 \times 10^{-3}$ & $1.6 \times 10^{0}$ & $3.5 \times 10^{-6}$ & $-2.0 \times 10^{2}$ & 0.38 \\
\hline
\end{tabular}

the subject age. Using our simulations from the $N=90$ subjects in this work, we attempted to fit a similar equation to the work of Scholkmann and Wolf to additionally incorporate head circumference and gender. The DPF values from each of the 320 10-5 positions on the head by 90 subjects were fit to a linear regression model using up to a third power of head circumference, age, and wavelength. A stepwise regression of the entire dataset was used to find the best set of covariates given the inclusion criteria of $p<0.05$ for the change in $F$-statistic of the increased model. Based on this, we model with a total of seven coefficients given by

$$
\begin{aligned}
\mathrm{DPF}= & \beta_{0}+\beta_{1}(\text { circ })+\beta_{2}(\text { circ })^{2}+\beta_{3}(\text { circ })^{3} \\
& +\beta_{4}\left\{\begin{array}{c}
1 \text { if female } \\
0
\end{array}\right\}+\beta_{5} \cdot(\text { age })+\beta_{6} \cdot(\lambda),
\end{aligned}
$$

where circ is the head circumference in millimeters, age is in months, and the wavelength is in nanometeres units.

\section{Disclosures}

None of the authors has any financial conflicts of interest to disclose related to this work.

\section{Acknowledgments}

The authors acknowledge the funding from the National Institutes of Health (No. R01MH107540; Perlman and RO1EB013210; Huppert).

\section{References}

1. F. F. Jobsis, "Noninvasive, infrared monitoring of cerebral and myocardial oxygen sufficiency and circulatory parameters," Science 198(4323), 1264-1267 (1977).
2. M. Mihara and I. Miyai, "Review of functional near-infrared spectroscopy in neurorehabilitation," Neurophotonics 3(3), 031414 (2016).

3. Y. Hoshi and Y. Yamada, "Overview of diffuse optical tomography and its clinical applications," J. Biomed. Opt. 21(9), 091312 (2016).

4. H. Obrig, "NIRS in clinical neurology-a 'promising' tool?" NeuroImage 85(Part 1), 535-546 (2014).

5. H. Obrig and A. Villringer, "Beyond the visible-imaging the human brain with light," J. Cereb. Blood Flow Metab. 23(1), 1-18 (2003).

6. D. A. Boas, A. M. Dale, and M. A. Franceschini, "Diffuse optical imaging of brain activation: approaches to optimizing image sensitivity, resolution, and accuracy," NeuroImage 23(Suppl. 1), S275-S288 (2004).

7. Y. Li et al., "The neural substrates of cognitive flexibility are related to individual differences in preschool irritability: a fNIRS investigation," Dev. Cognit. Neurosci. 25, 138-144 (2017).

8. S. B. Perlman et al., "fNIRS evidence of prefrontal regulation of frustration in early childhood," Neurolmage 85, 326-334 (2014).

9. S. Lloyd-Fox, A. Blasi, and C. E. Elwell, "Illuminating the developing brain: the past, present and future of functional near infrared spectroscopy," Neurosci. Biobehav. Rev. 34(3), 269-284 (2010).

10. R. N. Aslin and J. Mehler, "Near-infrared spectroscopy for functional studies of brain activity in human infants: promise, prospects, and challenges," J. Biomed. Opt. 10(1), 011009 (2005).

11. J. Gervain et al., "Near-infrared spectroscopy: a report from the McDonnell infant methodology consortium," Dev. Cognit. Neurosci. 1(1), 22-46 (2011).

12. R. N. Aslin, "Questioning the questions that have been asked about the infant brain using near-infrared spectroscopy," Cognit. Neuropsychol. 29(1-2), 7-33 (2012).

13. A. M. Fjell et al., "One-year brain atrophy evident in healthy aging," $J$. Neurosci. 29(48), 15223-15231 (2009).

14. C. Bonnery et al., "Changes in diffusion path length with old age in diffuse optical tomography," J. Biomed. Opt. 17(5), 056002 (2012).

15. M. S. Beauchamp et al., "The developmental trajectory of brain-scalp distance from birth through childhood: implications for functional neuroimaging," PLoS One 6(9), e24981 (2011).

16. M. Cope et al., "Methods of quantitating cerebral near infrared spectroscopy data," Adv. Exp. Med. Biol. 222, 183-189 (1988). 
17. S. B. Perlman et al., "Neural substrates of child irritability in typically developing and psychiatric populations," Dev. Cognit. Neurosci. 14, 71-80 (2015).

18. A. M. Dale, B. Fischl, and M. I. Sereno, "Cortical surface-based analysis. I. Segmentation and surface reconstruction," NeuroImage $\mathbf{9}(2), 179$ 194 (1999).

19. M. F. Glasser et al., "The minimal preprocessing pipelines for the Human Connectome Project," Neurolmage 80, 105-124 (2013).

20. F. Segonne et al., "A hybrid approach to the skull stripping problem in MRI," Neurolmage 22(3), 1060-1075 (2004).

21. Q. Fang and D. R. Kaeli, "Accelerating mesh-based Monte Carlo method on modern CPU architectures," Biomed. Opt. Express 3(12), 3223-3230 (2012).

22. Q. Fang, "Mesh-based Monte Carlo method using fast ray-tracing in Plucker coordinates," Biomed. Opt. Express 1(1), 165-175 (2010).

23. Q. Fang and D. A. Boas, Eds., "Tetrahedral mesh generation from volumetric binary and grayscale images," in ISBI'09 IEEE Int. Symp. on Biomedical Imaging: From Nano to Macro, IEEE (2009).

24. G. Strangman, M. A. Franceschini, and D. A. Boas, "Factors affecting the accuracy of near-infrared spectroscopy concentration calculations for focal changes in oxygenation parameters," Neurolmage 18(4), 865-879 (2003)

25. S. L. Jacques, "Optical properties of biological tissues: a review," Phys. Med. Biol. 58(11), R37-R61 (2013).

26. K. J. Friston, Statistical Parametric Mapping: the Analysis of Functional Brain Images, 1st ed., p. 647, Elsevier/Academic Press, Amsterdam, Boston (2007).

27. R. S. Desikan et al., "An automated labeling system for subdividing the human cerebral cortex on MRI scans into gyral based regions of interest," NeuroImage 31(3), 968-980 (2006).

28. N. Tzourio-Mazoyer et al., "Automated anatomical labeling of activations in SPM using a macroscopic anatomical parcellation of the MNI MRI single-subject brain," NeuroImage 15(1), 273-289 (2002).
29. T. J. Huppert, "Commentary on the statistical properties of noise and its implication on general linear models in functional near-infrared spectroscopy," Neurophotonics 3(1), 010401 (2016).

30. D. K. Joseph et al., "Diffuse optical tomography system to image brain activation with improved spatial resolution and validation with functional magnetic resonance imaging," Appl. Opt. 45(31), 8142-8151 (2006).

31. A. Duncan et al., "Measurement of cranial optical path length as a function of age using phase resolved near infrared spectroscopy," Pediatr. Res. 39(5), 889-894 (1996).

32. C. E. Cooper et al., "The noninvasive measurement of absolute cerebral deoxyhemoglobin concentration and mean optical path length in the neonatal brain by second derivative near infrared spectroscopy," Pediatr. Res. 39(1), 32-38 (1996).

33. P. van der Zee et al., "Experimentally measured optical pathlengths for the adult head, calf and forearm and the head of the newborn infant as a function of inter optode spacing," Adv. Exp. Med. Biol. 316, 143-153 (1992).

34. M. Essenpreis et al., "Spectral dependence of temporal point spread functions in human tissues," Appl. Opt. 32(4), 418-425 (1993).

35. A. Duncan et al., "Optical pathlength measurements on adult head, calf and forearm and the head of the newborn infant using phase resolved optical spectroscopy," Phys. Med. Biol. 40(2), 295-304 (1995).

36. H. Zhao et al., "Maps of optical differential pathlength factor of human adult forehead, somatosensory motor and occipital regions at multiwavelengths in NIR,” Phys. Med. Biol. 47(12), 2075-2093 (2002).

37. F. Scholkmann and M. Wolf, "General equation for the differential pathlength factor of the frontal human head depending on wavelength and age," J. Biomed. Opt. 18(10), 105004 (2013).

Biographies for the authors are not available. 